\title{
BEHAVIORAL AND HORMONAL FLEXIBILITY ACROSS LIGHT ENVIRONMENTS IN GUPPIES (POECILIA RETICULATA)
}

\author{
A Thesis \\ presented to \\ the Faculty of California Polytechnic State University, \\ San Luis Obispo
}

\author{
In Partial Fulfillment \\ of the Requirements for the Degree \\ Master of Science in Biological Sciences
}

by
Julia C. Walz
August 2014 
(C) 2014

Julia C. Walz

ALL RIGHTS RESERVED 
TITLE:

Behavioral and hormonal flexibility across light environments in guppies (Poecilia reticulata)
AUTHOR:
Julia C. Walz

DATE SUBMITTED: August 2014

COMMITTEE CHAIR: Gita R. Kolluru, PhD

Associate Professor of Biological Sciences

COMMITTEE MEMBER: Francis X. Villablanca, PhD

Professor of Biological Sciences

COMMITTEE MEMBER: Christy Strand, PhD

Associate Professor of Biological Sciences 


\begin{abstract}
Behavioral and hormonal flexibility across light environments in guppies (Poecilia reticulata)

Julia C. Walz
\end{abstract}

Behavior may be dramatically influenced by changing environments, and differences in light intensity among environments may have important behavioral consequences. One approach to understanding changes in behavior is by studying behavioral syndromes, suites of correlated behaviors reflecting among individual consistencies in behavior expressed across behavioral situations (e.g., correlations between antipredator behaviors in different habitats), or across behavioral contexts (e.g., correlations among feeding, antipredator, or mating behavior) (Sih et al. 2004a). Guppies (Poecilia reticulata) provide a great model system to study behavior. Guppies are small, freshwater tropical fish that inhabit still pools in swift-flowing streams, and the backwaters of small rivers in mountain forest areas of Trinidad (Houde 1997; Magurran 2005). In this study I addressed the following questions using three low predation populations of guppies: 1) Do guppies display a behavioral syndrome for aggression and/or courtship across light situations?; 2) Are there mean level changes in aggression or courtship across light situations?;

3) Are mean level changes influenced by differing social environments that include or lack sexually receptive females?; 4) Are there correlations between behavior and the androgen hormones testosterone and 11-ketotestosterone?; 5) Are there correlations between behavior and the stress hormone cortisol?; 6) Are there mean level changes in hormone release rates across light environments?; and 7) Is flexibility in hormone release rates influenced by social environments that include or lack sexually receptive females? Guppies exhibit behavioral syndromes for both aggression and courtship. Furthermore, guppies exhibit behavioral flexibility for both aggression and courtship, but only in social environments that include sexually receptive females. I found no correlations between behavior and androgen hormones. I also did not find any correlations between behavior and the stress hormone cortisol. Furthermore, I did not find any mean level changes in hormone release rates across light environments. Interestingly, cortisol levels were higher in social environments in which sexually receptive females were absent. Many studies have looked at how the environment influences courtship behavior in guppies, especially employing high predation populations, but few studies have examined aggressive behavior or behavior in general with low predation populations. Furthermore, few studies have determined the role social environments play, and how hormones may interact with behavior. This study is important because it helps illuminate how low predation populations deal with changes in light intensity environments, and adds to what we understand about guppy behavior in general.

Keywords: Behavior, guppies, light environment, flexibility, behavioral syndromes, hormone 


\section{ACKNOWLEDGMENTS}

I am extremely grateful to Gita Kolluru, my committee chair, and to my committee members, Francis Villablanca and Christy Strand for all of their generous help with this thesis project. I am very grateful to Ryan Earley and Amanda Hanninen for help on hormone collection and extractions. I am grateful to Samantha Alburn Rebecca Nuffer, Brandy Kolbuch, Shelly Gupta, Alexandra Barbella, and Kate Downey for their assistance with data collection and fish care, all members of the Kolluru fish lab at California Polytechnic State University for assistance with maintenance of fish colonies, Gregory F. Grether and Brian Smith for providing us with fish, Doug Brewster and Nancy Reid for assistance with lab facilities, and the California State University Extramural Funding Initiative, State Faculty Support Grants and California Polytechnic State University, San Luis Obispo, for funding. This research was conducted with IACUC approval. 


\section{TABLE OF CONTENTS}

Page

LIST OF TABLES $\quad$ ix

LIST OF FIGURES $\quad$ xi

\section{CHAPTER}

I. GENERAL INTRODUCTION 1

Behavioral Flexibility 1

Behavioral Syndromes $\quad 2$

$\begin{array}{ll}\text { The Study System } & 6\end{array}$

$\begin{array}{ll}\text { Predation Cues and Prey Response } & 9\end{array}$

$\begin{array}{ll}\text { Predation, Risk, and the Guppy System } & 13\end{array}$

Light Intensity and Relationships with Predation Risk in the Guppy $\begin{array}{ll}\text { System } & 15\end{array}$

II. GUPPY COURTSHIP AND AGGRESSION ACROSS LIGHT ENVIRONMENTS IN DIFFERING SOCIAL ENVIRONMENTS: AN INVESTIGATION INTO BEHAVIORAL SYNDROMES 19

$\begin{array}{ll}\text { Introduction } & 19\end{array}$

$\begin{array}{ll}\text { Methods } & 23\end{array}$

$\begin{array}{ll}\text { Outline of Experimental Design } & 23\end{array}$

$\begin{array}{ll}\text { Study Populations } & 23\end{array}$

$\begin{array}{ll}\text { Laboratory Setup } & 24\end{array}$

$\begin{array}{ll}\text { Focal Behavioral Observations } & 26\end{array}$

$\begin{array}{ll}\text { Behavioral Variables } & 30\end{array}$

$\begin{array}{ll}\text { Data Analysis } & 30\end{array}$

Results 31 
Spearman's Rank Correlations $\quad 32$

$\begin{array}{ll}\text { Discussion } & 34\end{array}$

III. GUPPY AGGRESSION AND COURTSHIP AND THE RELATIONSHIP OF THESE BEHAVIORS WITH CORTISOL, TESTOSTERONE, AND 11-KETOTESTOSTERONE ACROSS LIGHT ENVIRONMENTS IN THE PRESENCE AND ABSENCE OF SEXUALLY RECEPTIVE FEMALES

$\begin{array}{ll}\text { Introduction } & 57\end{array}$

$\begin{array}{ll}\text { Methods } & 63\end{array}$

$\begin{array}{ll}\text { Outline of Experimental Design } & 63\end{array}$

$\begin{array}{ll}\text { Study Populations } & 64\end{array}$

$\begin{array}{ll}\text { Laboratory Setup } & 65\end{array}$

Stable Social Group Focal Observations $\quad 66$

$\begin{array}{ll}\text { Behavioral Variables (Stable Group) } & 68\end{array}$

$\begin{array}{lr}\text { Challenge Situation Tank Focal Observations } & 68\end{array}$

$\begin{array}{ll}\text { Behavioral Variables } & 72\end{array}$

$\begin{array}{ll}\text { Hormone Sampling } & 72\end{array}$

$\begin{array}{ll}\text { Data Analysis } & 76\end{array}$

$\begin{array}{ll}\text { Results } & 78\end{array}$

Effects of the light level and Female Absent social environment on behavior

Effects of the light level and Female Present social environment on behavior

Relationship between hormone levels and behavior in Stable

Social Group observations 
Relationship between hormones and behavior in Social Challenge observations

Effects of experimental conditions on circulating hormone levels of males

Discussion 


\section{LIST OF TABLES}

Tables

Pages

1

Description of the male behaviors recorded in the Social Challenge focals.

Results of principal component analysis on behavioral measures recorded in Female Absent trials in High Light situations. Component loadings that are bold are significant loadings $>0.4$.

Results of principal component analysis on behavioral measures recorded in female absent trials in Low Light situations. Component loadings that are bold are significant loadings $>0.4$.

Results of principal component analysis on behavioral measures recorded in female present trials in high light situations. Component loadings that are bold are significant loadings $>0.4$.

Results of principal component analysis on behavioral measures recorded in female present trials in low light situations. Component loadings that are bold are significant loadings $>0.4$.

Spearman's Rank correlation data for PCA results for male guppy behavior in Female Absent trials between High light and Low Light Situations. The * indicates statistically significant correlations.

Spearman's Rank correlation data for PCA results for male guppy behavior in Female Present trials between High light and Low Light Situations. The * indicates statistically significant correlations. Bonferroni adjusted significance for 3 tests is alpha at 0.0166 .

Spearman's rank results for female absent trials by population (AR: Aripo; MR: Marianne; SC: Small Crayfish) results. There was no significant population results after a strict Bonferroni correction $(\alpha=0.0166)$.

Spearman's rank results for female present courtship component by population (AR: Aripo; MR: Marianne; SC: Small Crayfish). The MR population results after a strict Bonferroni correction $(\alpha=0.0166)$ were significant.

Spearman's rank results for female present aggression component by population (AR: Aripo; MR: Marianne; SC: Small Crayfish). The Aripo population results after a strict 
11 Description of the behaviors recorded in the Stable Group Tank observations.

12 Description of the male behaviors recorded in the Challenge Situation Tank Observations.

13 Male behavior in Female Absent social environments.

14 Male behavior and Female Response to Male behavior in Female Present social environments. * indicates significant results after Bonferroni correction. ** indicates significance before Bonferroni correction.

Analysis of covariance of population, light level, day, and mass as a function of female absent and female present behavioral indices. ** indicates a significant result before Bonferroni correction. * indicates a significant result after Bonferroni correction. 


\section{LIST OF FIGURES}

Combinations of how behavioral syndromes can combine with flexibility. Where a behavioral syndrome exists, the rank order of the individuals $\mathbf{1}$ through $\mathbf{4}$ is maintained for a given behavior between environmental conditions (Panels A and B). Where a behavioral syndrome does not exist the rank order is not maintained (Panels C and D). Flexibility is expressed when the behavior is shifted consistently in the same direction in different environmental conditions (Panel A and C). Panel A represents a situation in which flexibility and a syndrome both exist. Where flexibility does not exist, the behavior of the individuals either does not shift between environmental conditions or shifts randomly such that some individuals act in a completely different way than other individuals under the same conditions (Panels B and D).

The set up of the two observation tanks. Fish were placed in one of the two identical tanks pictured. The small caddies seen in front of Tank 1 (the observation tank on the left) were used as acclimation chambers. The small table in front held the computer used for recording behaviors. To the right of the computer is the piece of shade cloth used to create low light levels on the tank. The piece was laid across the top of two tanks, effectively blocking the light created from the light sources above the tanks (not seen in this image). The black curtain to the far right could be pulled around to separate the observation tanks from the rest of the room.

The lab room at Cal Poly, San Luis Obispo. Home Tanks are on the racks on the right side of the image and the observation tanks are on the left side of the image, near the black curtain.

Reaction norm plot for female absent aggression component between high and low light levels. Each male's component score in high and low light is represented by a particular line with the legend showing Male ID numbers.

Reaction norm plot for female present courtship component between high and low light levels. Each male's component score in high and low light is represented by a particular line with the legend showing Male ID numbers.

Reaction norm plot for female present aggression/competition component between high and low light levels. Each male's 
component score in high and low light is represented by a particular line with the legend showing Male ID numbers.

Reaction norm plots by population (A: Aripo; B: Marianne; C: Small Cray) for female absent male -male aggression component between high and low light levels. Each male's component score in high and low light is represented by a particular line with the legend showing Male ID numbers.

Reaction norm plots by population (A: Aripo; B: Marianne; C: Small Cray) for female present courtship component between high and low light levels. Each male's component score in high and low light is represented by a particular line with the legend showing Male ID numbers.

Reaction norm plots by population (A: Aripo; B: Marianne; C: Small Cray) for female present aggression/competition component between high and low light levels. Each male's component score in high and low light is represented by a particular line with the legend showing Male ID numbers.

10 Mean male aggressive behavioral index (+/- standard error) in Female Absent social situations $(n=48)$.

Mean male-male chases performed (+/- standard error) in Female Absent social situations $(n=48)$.

Mean male courtship behavioral index (+/- standard error) in Female Present social situations $(n=48)$.

Mean male competition behavioral index (+/- standard error) in Female Present social situations $(n=48)$.

Mean female response to male courtship (+/- standard error) in Female Present social situations $(n=48)$.

Mean sigmoid rates of males (+/- standard error) in Female Present social situations.

Mean male courtship behavioral index (+/- standard error) in Female Present social situations by population (AR: Aripo River; MR: Marianne River; SC: Small Crayfish).

Mean male competition behavioral index (+/- standard error) in Female Present social situations by population (AR: Aripo River; MR: Marianne River; SC: Small Crayfish). 
18 Mean female response to male courtship (+/- standard error) in Female Present social situations by population (AR: Aripo River; MR: Marianne River; SC: Small Crayfish).

Mean testosterone release rates $(\mathrm{pg} / \mathrm{ml})(+/-$ standard error) by population (AR: Aripo River $(\mathrm{n}=36)$; MR: Marianne River $(n=36) ;$ SC: Small Crayfish River $(n=36))$.

20 Mean cortisol release rates $(\mathrm{pg} / \mathrm{ml})$ (+/- standard error) in two different social situations (Female Absent $(n=48)$ and Female Present $(n=48))$. 
I.

\section{GENERAL INTRODUCTION}

\section{Behavioral Flexibility}

The phenotype is the physical manifestation of an organism's genotype (Sih et al. 2004b; Ghalambor et al. 2010). The capacity for a given genotype to produce different phenotypes in response to different environmental conditions is termed phenotypic plasticity (West-Eberhard 2003; Sih et al. 2004b; Ghalambor et al. 2010). The magnitude of plasticity may vary based on environmental variation and can be measured on a continuous scale of responses (Ghalambor et al. 2010). If phenotypes are relatively fixed manifestations of the genotype, this means that the environment has very little if any influence on phenotype, whereas a plastic phenotype results if phenotype is influenced by environmental factors (Ghalambor et al. 2010).

Plasticity in general is expected to evolve when costs to being plastic are low, the ability for the individual animal to gage conditions in the environment is good (learning may increase this ability), and the environment varies with time (Via and Lande 1985; Via 1987; Moran 1992; Komers 1997; Ghalambor et al. 2003; Sih et al. 2004b; Ghalambor et al. 2010). Adaptive plasticity refers to plasticity that is beneficial to the fitness of an organism and maintained by selection (Ghalambor et al. 2007). Adaptive plasticity is expected to evolve if 1) environments are variable, 2) organisms can reliably respond to environmental cues, 3) there is differing selective pressure on phenotypes in different environmental conditions, and 4) there is no single phenotype that exhibits its highest fitness across all environments (Via and Lande 1985; Via 1987; Moran 1992; Komers 1997; Ghalambor et al. 2003; Sih et al. 2004b; Ghalambor et al. 2010). 
Behavior is a measurable phenotypic expression of genotype, and is more reversible, flexible, and highly amendable compared to some morphological and physiological traits (Sih et al. 2004b; Ghalambor et. al. 2010). Behavioral phenotypes can be highly variable within populations, and individuals may alter behavioral responses to environmental stimuli frequently throughout life, often as a manifestation of learning, or may express relatively fixed behavioral responses, such as the fixed action patterns described by Lorenz in1965 (Sih et al. 2004b; Ghalambor et al. 2010). Behavioral flexibility allows for optimal changing behavior in an individual, and because of this flexibility, individuals can respond to challenges with appropriate behaviors without overreacting and wasting time and energy, or under reacting and risking injury or death (West-Eberhard 1989; Alcock 2005; Wright et al. 2010). Insight into how behavioral phenotypes may be correlated across environmental conditions, a phenomenon known as "behavioral syndromes", may shed light on the evolution of phenotypic plasticity and its constraints by various factors (Sih et al. 2004a; Sih et al. 2004b).

\section{Behavioral Syndromes}

How animals respond to changing environments is an important field of study in behavioral ecology (Endler 1995; Houde 1997; Magurran 2005; Sih et al. 2010; Conrad et al. 2011). A growing number of studies suggest that animals may exhibit individual variation in behavioral tendencies, such that a given individual has a behavioral type (or personality) such as being bold or shy (See Sih et al. 2004a; Sih et. al. 2004b; Sih et al. 2010; and Conrad et al. 2011). A "behavioral type" is defined as within- individual consistency in behavior, such that the individual exhibits consistent behavior across observations and the behavior can be measured independent of the behaviors of others 
(Gosling 2001; Sih et al. 2004a; Sih et al. 2004b; Sih et al. 2010). It is clear that these behavioral types influence an individual's behavioral responses to a variety of environmental conditions (Sih et al. 2004a).

If behavioral types are consistent across different environmental conditions such that a rank order of individuals is maintained, then it is termed a behavioral syndrome. Behavioral syndromes have been identified in a variety of taxa (see reviews by Wilson et al. 1994; Gosling 2001; Sih et al. 2004a; Conrad et al. 2011). Behavioral syndromes describe suites of correlated behaviors reflecting between individual consistencies in behavior expressed within a behavioral context (context refers to a functional behavioral category - for example, mating and feeding are different contexts), also known as a situation (e.g., correlations between antipredator behaviors in different habitats), or across behavioral contexts (e.g., correlations among feeding, antipredator, or mating behavior) (Sih et al. 2004a). Between individual consistency (or rank order consistency) is the tendency of individuals to exhibit a behavioral type (within-individual consistency) such that each individual generally retains its rank order of behavior among the other individuals in the group across situations or contexts (Sih et al. 2004a; Sih et al. 2004b; Sih et al. 2010). Populations of individuals, individual species, and even groups of species can exhibit behavioral syndromes, with each individual, population or species showing a behavioral type within the larger syndrome (Sih et al. 2004a; Sih et al. 2004b; Sih et al. 2010).

Traits that are governed by behavioral syndromes in a population can still exhibit behavioral flexibility (Sih et al. 2004a; Sih et al. 2004b; Sih et al. 2010). If individuals shift their behavior among situations or contexts in response to environmental 
differences, but still maintain rank order in levels of behavior, then they are expressing plasticity as well as a behavioral syndrome. Figure 1 shows how behavioral syndromes may combine with adaptive plasticity. Panel A illustrates a population that shows a mean flexibility in their courtship display rate in response to light environment, but also exhibits a behavioral syndrome in that rank order is maintained among the individuals of the population. Behavioral syndromes can exist without a population showing mean flexibility in behavior (illustrated in Panel B). When individuals do not maintain rank order but do show flexibility, plasticity without a syndrome is shown (Panel C). And finally you may see neither adaptive plasticity, or a syndrome (Panel B).

Behavioral syndromes have the potential to explain maladaptive behavior. Because behavioral syndromes may describe consistency in behavior across contexts or across situations, what an individual does in one context or situation may be coupled to what it does in another context or situation. For example, the most aggressive, showy or bold individual in a low predation situation may also be the most aggressive, showy or bold individual in the high predation situation. Thus behavior may be constrained by a syndrome such that the syndrome may result in behavior that is carried over from one context or situation where it is adaptive to another where it would be considered maladaptive (Sih et al. 2004 a; Sih et al. 2004b; Sih et al. 2010). For example, if aggressive behavior is constrained by a syndrome than an individual that may be highly aggressive towards a territorial intruder (adaptive) may also carryover that aggression into a mating context and be highly aggressive toward a potential mate which may have direct affect on that individuals fitness (maladaptive). 
A good way to approach behavioral syndromes is to focus on the proximate and ultimate explanations for them. Niko Tinbergen, a Nobel-prize winning ethologist, suggested behaviorists ask themselves four fundamental questions that consider proximate (how) and ultimate (why) explanations for a behavior (Blumstein and Fernandez-Juricic 2004; Alcock 2005; Buchholz 2007). These questions are: 1.What is the mechanism that controls the behavior? 2. What is the ontogeny of the behavior? 3 . What is the function of the behavior? 4 . What is the phylogenetic origin of the behavior (Alcock 2005; Buchholz 2007)? The proximate questions (\#'s 1and 2 in the list above) address the physical mechanisms that underlie how an individual behaves the way that it does, and the ultimate questions (\# 3 and 4) ask what the history and adaptive value of the behavior may be (Alcock 2005; Buchholz 2007). For a behavioral syndrome, the proximate explanations refer to the physical mechanisms that underlie a syndrome, whereas ultimate explanations address why the syndrome evolved.

A proximate explanation for the existence of a behavioral syndrome is that behavior across contexts or situations is linked via hormonal control (Sih et al. 2004b; Ketterson et al. 2009). For example, if testosterone levels influence aggression, a behavioral syndrome for aggression may be driven by variation among individuals in their exclusive levels of circulating testosterone (Sih et al. 2004a; Sih et al. 2004b; Ketterson et al. 2009; Conrad et al. 2011). By studying underlying proximate mechanisms for behavioral syndromes, we can look for explanations for syndromes that are relatively stable over time and potentially related to fixed hormonal pathways. Ultimate explanations for behavioral syndromes include: 1) the costs of switching behavioral types outweigh the benefits of specializing in a particular type, especially if 
the environmental conditions are hard to assess, and 2) individuals do best when they continue to do what they have done consistently, which suggests the benefits of learning a "style" (Sih et al. 2004a; Sih et al. 2004b). The existence of behavioral syndromes reminds us that organisms need to be studied as a whole rather than as a system of nonintegrated parts (Sih et al. 2004a; Sih et al. 2004b).

To establish a behavioral syndrome for a given trait, observations of behavior in different contexts or situations need to be obtained for each set of individuals. In this study I examined aggression and courtship in male guppies (Poecilia reticulata) in social environments with and without sexually receptive females. I recorded the behavior of the same males in two light situations that may be perceived by guppies as differing in predation risk, high light (high risk) and low light (low risk), to determine if males exhibit behavioral syndromes for courtship and aggression across light intensity environments.

\section{The Study System}

Guppies (Poecilia reticulata) are small, freshwater tropical fish in the live-bearing family Poeciliidae. There are 22 genera in the sub-family Poeciliinae, which includes guppies, and 43 species in the genus Poecilia (Moyle and Cech 2000). Poecillid fish, including the guppy, are relatively small, rarely exceeding $10 \mathrm{~cm}$ in standard length, are adapted to warm, fresh to slightly brackish water, and have a broad tolerance for variation in temperature and salinity (Moyle and Cech 2000). Poecillid fish are also characterized by internal fertilization achieved via the gonopodium (male sex organ), and viviparity (Moyle and Cech 2000). 
Guppies inhabit still pools in clear, swift-flowing streams and the edges and backwaters of small rivers in mountain forest areas, though they can tolerate a wide range of stream and river conditions (e.g., brackish or polluted waters; Houde 1997). They are omnivorous, opportunistic feeders, consuming algae, insect larvae, other invertebrates, benthic detritus, the eggs and young of Rivulus hartii, and their own young (Houde 1997).

Although there is some debate about the complete native range of guppies, they are considered native to Trinidad and Tobago, Venezuela, Guyana, and Suriname. They are quite possibly native to Barbados, Cuba, and Grenada, although these may have been early introductions or invasions (Houde 1997; Magurran 2005). Guppies can now be found on every continent except Antarctica (Magurran 2005), and are therefore one of the most widely distributed tropical fish (Magurran 2005). Because of their role in mosquito control and their consequent introduction throughout much of the world, guppies have displaced native fish and are a major invasive problem in several areas, including parts of Asia and Australia (Houde 1997; Lindholm et al.1999; Magurran 2005). Guppies are also extremely popular pet store fish and have been selectively bred for over a 100 years for various aspects of their morphology, including elaborate colors and caudal fins (Houde 1997; Magurran 2005).

Guppies have been extensively studied in the fields of genomics, evolution, and behavioral ecology, and much is known about their natural history (see Houde 1997; Magurran 2005). In a laboratory setting, guppies are ideal study subjects because of the relative ease in rearing and caring for them (Magurran 2005). Guppies are best categorized as having a promiscuous mating strategy (Houde 1997). Males are capable of 
mating several times a day if there are receptive females available, whereas females can mate with two or three males each time they are receptive, which occurs for only two to three days of every 25-30 day reproductive cycle (during the postpartum period) and when they reach sexual maturity (10 to 20 weeks of age; Houde 1997). However, female guppies can store sperm for up to 8 months, to fertilize several successive broods of young and produce quite a large number of young - up to 20 or more - per litter (Houde 1997; Magurran 2005; Lopez-Sepulcre et al. 2013).

Guppies display pronounced sexual dimorphism in coloration. Females are a pale tan color, whereas males display a wide range of colors from contrast silver and black lines and dots to bright spot areas of orange, yellow, red, blue and green (Grether 2000). The color saturation (chroma) of orange spots is carotenoid based and the carotenoid pigments contributing to the chroma of the spots must be acquired from their diet (Goodwin 1984; Kodric-Brown 1989; Grether 2000). Female choice is based on color patterns, predominantly a male's orange spot area and distribution, orange spot chroma, and body shape (Houde 1997). Furthermore, investigations into geographic variation in female preferences established that females respond more intensely to males native to their own streams versus non-native males, prefer orange and dislike bronze-green coloration, and have a preference for larger caudal fin size (Endler and Houde 1995). However there is extreme variation in these preferences among populations (Endler and Houde 1995; Brooks and Endler 2001).

Male guppies exhibit three mating tactics: courtship display, sneak copulation, and competition (Houde 1997; Magurran 2005; Kolluru and Grether 2005). The courtship display is sometimes followed by copulation with the female's cooperation, whereas 
sneak copulation is not preceded by display and is presumably achieved without female cooperation (Houde 1997). Competition involves the disruption of courtship by another male and mating with the courted female by the non-courting disruptive male (Houde 1997; Jirotkul 2000; Magurran 2005). The courtship display is characterized by the "sigmoid" quiver (Houde 1997). A male assumes an S-shape with his body in front of a female and then quivers his body, making his colors shimmer and presumably becoming more visible (Houde 1997). Males may display up to 2.7 times per minute and attempt a mating 0.5 times per minute (Houde 1997). Courtship display increases the risk of predation because courting guppies can be seen from as far as 2 meters away (Houde 1997; Jirotkul 2000). The sneak copulation is often referred to as "gonodopodal thrusting" and is usually attempted from the side of or behind a female (Houde 1997). Sneak copulations are hypothesized to be less risky in higher predation sites because they are less conspicuous then the full courtship display (Magurran and Seghers 1990; Houde 1997). Competition is often referred to as either "male-male competition" or a "mating attempt with a female that is being courted by another male" (Houde 1997; Jirotkul 2000; Kolluru and Grether 2005). Male guppies may switch among mating tactics throughout their lifetimes, and mating tactics can be influenced by many factors, including female receptivity, behavior of other males, sex ratio, population density, and predation risk of the environment (Magurran and Seghers 1990; Endler 1995; Jirotkul 1999a; Jirotkul 1999b; Jirotkul 2000).

\section{Predation Cues and Prey Response}

Predation is one of the strongest selective pressures on morphological, physiological, and behavioral traits of prey species (Alcock 2005). Predation has been 
shown to affect foraging behavior, habitat use, aggression, boldness, dominance, and social interactions among a variety of taxa (Endler 1987; Lima and Bednekoff 1999; Nonacs and Blumstein 2010; Kelley and Brown 2011). Prey individuals are expected to optimize the trade-offs between fitness-enhancing activities and predation risk, but this relies on several key abilities, including being able to acquire reliable and accurate information about predation risk and, once this information is acquired, implement an appropriate response (Nonacs and Blumstein 2010).

Animals can gather information about predation risk via visual cues, either directly by engaging in "predator inspection" (a behavior characterized by an individual or group approaching a potential predator to ascertain its identity and its potential risk), by observing the behavior of conspecifics, or by recognizing environments that are known to be risky, such as environments with high visibility (Endler 1987; Dugatkin and Godin 1992; Reynolds et al. 1993; Magurran 2005).

The response to predation risk involves a variety of reactions, including the physiological stress response, involving increases in circulating levels of the stress hormone cortisol (Reid et al. 1998). Cortisol is the primary physiological end-product in the overall stress response of many animals including guppies (Martinez-Porchas et al. 2009; Conrad et al. 2011). Cortisol release in fish primarily causes gylcogenolysis and gluconeogensis processes which increases glucose levels for energy and modulate cardiovascular and respiratory functions (Reid et al. 1998; Martinez-Porchas et al. 2009).The hormonal changes may lead to shifts in behavior appropriate to respond to risk (Magurran and Seghers 1990; Martinez-Porchas et al. 2009). 
Environments often harbor predator cues such as a predator's scent or other chemical cues associated with the predator on a continuous basis. How, then, does an animal decide when to respond? Anti-predator behavior may commence upon the detection of threshold levels of predatory cues (Kelley and Brown 2011). The risk allocation hypothesis suggests that temporal variation in predation risk should influence the intensity of prey vigilance, foraging, and other behaviors depending on the extent that predators are present and the degree of risk posed by a particular predator (Lima and Bednekoff 1999; Van Buskirk et al. 2002; Ferrari et al. 2009). In environments where predators are relatively scarce, prey can forage during times that no predators are detected and then respond strongly when predators are present. Alternatively, in environments where predators are always present, prey species may need to take risks and forage even when it is not very safe. It follows that in areas of high predation risk, individuals should require higher levels of predation cues to stimulate anti-predator behavior, that the antipredator response should be stronger when detected predation cues are more sporadic, and that vigilance should be highest in situations in which high risk is rare (Lima and Bednekoff 1999; Ferrari et al. 2009; Nonacs and Blumstein 2010; Kelley and Brown 2011). Brown et al. (2005a) tested this hypothesis in a poeciliid fish, the bishop livebearer (Brachyrhaphis episcopi), and showed that indeed high and low risk populations differed in their stress response to predation cues, with high risk populations requiring higher levels of cues to induce the stress response, as well as being better able to cope with stress.

Other hormonal responses to changing environments may include androgen hormones. Androgen hormones, including testosterone and especially its derivative 11- 
ketotestosterone (11-KT), which appears to be the major androgens in teleost fish, stimulate masculine traits including male reproductive and competitive behaviors (see Borg 1994 for a review). Changes in male behavior such as courtship in response to predation cues may be correlated with changes in androgen release rates. Extensive studies involving three-spined stickleback show that castration, which results in reduction of circulating androgen levels, results in reduced courtship and competitive behavior (Hoar 1962; Wai and Hoar 1963; Borg 1987). Implants of 11-KT and testosterone restored male behaviors, with 11-KT being more effective than testosterone (Borg 1987). In a separate experiment, bluegill sunfish increased antipredator aggressiveness with 11KT implants while testosterone implants were less effective (Kindler et al. 1991). Androgens, therefore, may be important hormones in mediating behavior in response to changing predation risk environments.

As stated above, animals respond in multiple fixed or plastic ways to predation risk. Some animals hide or flee, others heighten their vigilance, create social defense mechanisms such as schooling, shoaling or herding, or employ morphological defenses such as shells (Lima 1992; Lima 1998; Templeton and Shriner 2004; Alcock 2005). Predation creates a variety of cost benefit trade-offs, including how to allocate energy to defense or foraging and whether to court or sneak copulations. Natural selection acts strongly on anti-predatory behavior and predation risk may be the most important factor in studying life history strategies in any organism (Nonacs and Blumstein 2010). 


\section{Predation, Risk and the Guppy System}

Understanding predation pressure on guppy populations is fundamental to understanding much of guppy population biology. Variation in predation risk among guppy streams in Trinidad has been correlated with divergence in behavior, morphology, male coloration, density, and life history traits (reviewed by Endler 1995; Rodd and Sokolowski 1995; Magurran 2005). Therefore, guppy field research has been particularly focused on the influence of predation regimes on trait evolution.

The two most commonly studied predators of guppies are the killifish (Rivulus hartii) and the cichlid (Crenicichla alta; Endler 1995). Endler (1995) defines high predation populations as those subjected to predation by $C$. alta, and low predation populations as those subjected to $R$. hartii and the prawn, $M$. crenulatum. Other guppy predators include arial predators such as bats (Noctilio leporinus) and birds (kingfishers and kiskadees) (Reznick and Endler 1982; Templeton and Shriner 2004). In any given drainage, the upstream reaches of the stream contain relatively few predatory fish, typically limited to $R$. hartii, because of the difficulty of large fish colonizing areas above waterfalls (Liley and Seghers 1975; Templeton and Shriner 2004). In contrast, downstream areas experience a wide variety of fish predators, particularly C. alta (Liley and Seghers 1975; Templeton and Shriner 2004). Endler (1995) suggests that predation accounts for $70 \%$ of the variation in guppy life history patterns among populations. Higher predation rates leading to greater guppy mortality lead to evolutionary changes such as early maturation, smaller body size with larger, more fusiform body length-toheight ratio, and higher fecundity (Endler 1995). Reznick and Endler (1982) suggested that both the intensity of predation and differential predation on different size classes of 
guppies could cause life history divergence among populations subjected to different predation regimes. Indeed, significant divergence in demographic traits among guppy populations, including differences in size-specific fecundity, size at reproductive maturation, size of mature males, offspring size, interbrood interval, and percentage of female body weight devoted to embryos, is a result of varying mortality risk due to predation (Reznick and Endler 1982; Endler 1995; Rodd and Reznick 1997).

Interestingly, guppies in another high and low-predation site dominated by a completely different set of predators exhibit similar life history and demographic patterns as the ones dominated by $R$. hartii and $C$. alta, suggesting that predator induced mortality selects for life history evolution (Reznick et al. 1996). Endler (1995) and Reznick et al. (1997) point out that guppies can respond to differences in predation intensity (as seen in the evolution of male color intensity and various life history traits) in as little as 6 to 40 generations, covering a span of only 2 to 11 years. Varying life history patterns and demography among populations continues to be a subject of research in the guppy system.

Mating tactics are also affected by predation pressure. Magurran and Seghers (1990) demonstrated that male guppies from high-predation populations are more likely to employ sneak copulation than court, even in the absence of predators, and suggest that males should modify courtship behavior when faced with a predation threat in a manner dependent on the predation pressure of the population. They subsequently demonstrated that Lower Aripo River (low-predation) guppies showed a decrease in the frequency of courtship displays and an increase in sneak attempts when predators were present, but that no such correlation is shown for Upper Aripo River (high-predation) guppies 
(Magurran and Seghers 1990). Endler (1995) discussed several environmental gradients including light levels, food availability, and predation regime that could act on male mating tactics. He suggested that increased light intensity and wavelengths causes increased food availability but also increased visibility (Grether et al. 2001), and although increased food should allow more courtship, males decreased courtship displays, possibly because of high predation risk (Endler 1995).

\section{Light Intensity and Relationships with Predation Risk in the Guppy System}

Color patterns and the courtship display are the most important parts of the conspicuous guppy sexual display (Houde 1997; Magurran 2005). Sexual displays can also attract predators (Zuk and Kolluru 1998). The conspicuousness of signals to both predators and conspecifics is influenced by environmental conditions, including light conditions, because these conditions influence signal dissemination and perception (Endler 1987; Reynolds et al. 1993; Long and Rosenqvist 1998; Gamble et al. 2003; Archard et al. 2009). Variation in light conditions and subsequent visibility of conspicuous behavior could create trade-offs between the benefits of courtship for reproduction and the costs of attracting a predator (Archard et al. 2009).

Archard et al. (2009) found that guppy mating behavior, especially the courtship display, is correlated with both the quantity and quality of light. Males displayed more at lower light levels and at light levels simulating dawn and dusk than at higher light levels and those simulating levels at high noon (Endler 1987; Kolluru et al. 2007; Archard et. al 2009). Furthermore, Archard et al. (2009) determined that behavioral modification as a result of light level was not solely a time-of-day effect, but rather was a result of direct 
changes in the light levels (Archard et al. 2009). A similar study by Long and Rosenqvist (1998) that looked at changes in male guppy courting distance in response to light level showed that males courted at closer distances in low light situations (light that simulated dawn, dusk, and heavy canopy cover) than in high light situations. Changes in light caused direct changes in guppy behavior.

Endler (1987) pioneered studies of light intensity and predation on male courtship behavior. Endler (1987) showed that high light intensity corresponded to high perceived predation risk by guppies. Males cannot alter their color patterns to be less discernible to predators on a short time-scale, but they can change their behavior patterns. Endler (1987) concluded that males used visually prominent behavioral elements such as the courtship display less often under higher light levels that occurred during the middle of the day, and also in the presence of actual predators (Endler 1987). Furthermore, in a greenhouse experiment (with predators absent), Endler (1987) found that captive guppies originating from Trinidad and Venezuelan exhibited a diurnal pattern (dawn and dusk when light levels were low) of conspicuous displays, and this pattern was seasonal in nature, occurring only in summer and not the winter months when light intensity did not show a diurnal pattern. Males courted less and used visual signals less under higher light levels whether or not predators were present. Endler (1987) also showed that the pike cichlid (C. alta), the main predator in high predation populations, was more active and attacked guppies at a significantly higher intensity at midday (higher light conditions) than at dawn and dusk (lower light conditions). As predicted, guppies used less conspicuous behavior when $C$. alta was present. They increased their courtship displays to times or locations with lower light levels when it was harder for females and predators 
to discern color patterns (Endler 1987). In conclusion, perceived predation risk is extremely important, even overriding the best light conditions for female choice. Guppies tend to engage in the courtship display more at these lower light levels (Endler 1987).

Reynolds et al. (1993) examined the frequency of alternative mating tactics by males in varying light levels (low and high light) that simulated low and high predation risk. Reynolds et al. (1993) found that males displayed more frequently in low light versus high light, consistent with Endler (1987). He also found that larger males displayed less often than smaller males under high light and that larger males did not compensate with increased sneaking under high light levels. This suggests that the potential risk of predation in high light is particularly costly for larger, more conspicuous males, and that males are exhibiting individual variation in plasticity based on body size. Under low light levels, only males with long gonopodia were more likely to use sneak copulations. Reynolds et al. (1993) supported Endler's (1987) light environment findings that suggest that high light situations indicate high predation risk, when guppies should reduce their conspicuous signals. 
Figures:

\section{Adaptive Flexibility}

YES

NO

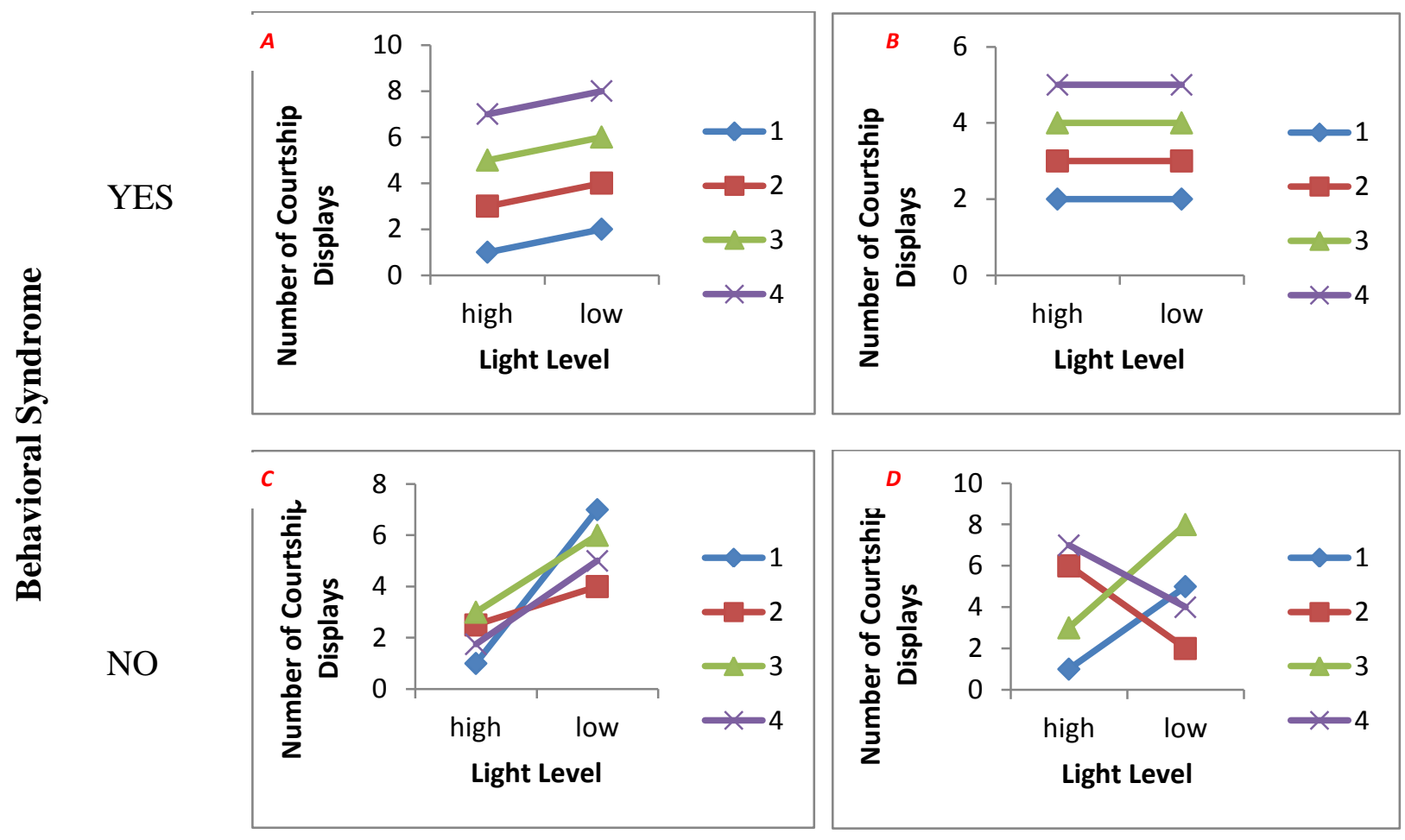

Figure 1: Combinations of how behavioral syndromes can combine with flexibility.

Where a behavioral syndrome exists, the rank order of the individuals 1 through $\mathbf{4}$ is maintained for a given behavior between environmental conditions (Panels A and B).

Where a behavioral syndrome does not exist the rank order is not maintained (Panels C and D). Flexibility is expressed when the behavior is shifted consistently in the same direction in different environmental conditions (Panel A and C). Panel A represents a situation in which flexibility and a syndrome both exist. Where flexibility does not exist, the behavior of the individuals either does not shift between environmental conditions or shifts randomly such that some individuals act in a completely different way than other individuals under the same conditions (Panels B and D). 
II.

\section{GUPPY COURTSHIP AND AGGRESSION ACROSS LIGHT ENVIRONMENTS IN DIFFERING SOCIAL ENVIRONMENTS: AN INVESTIGATION INTO BEHAVIORAL SYNDROMES}

\section{Introduction}

How animals respond to a changing environment is an important field of study in behavioral ecology (Endler 1995; Houde 1997; Magurran 2005; Alcock 2005; Sih et al. 2010; Conrad et al. 2011). Behavioral flexibility allows for optimal changing behavior in an individual, and because of this flexibility, individuals can respond to challenges with appropriate behaviors without overreacting and wasting time and energy, or under reacting and risking injury or death (West-Eberhardt 1989; Alcock 2005; Wright et al. 2010). Behavioral phenotypes can be highly variable among populations, and individuals may express and change their particular behavioral responses to environmental stimuli frequently throughout life, often in response to learning, or may express relatively fixed behavioral responses, such as the fixed action patterns described by Lorenz in 1965 (Sih et al. 2004b; Ghalambor et al. 2010). A growing number of studies illustrate that animals may exhibit behavioral types such as bold or shy (Huntingford 1976; Wilson et al. 1993; Gosling 2001; Bell and Sih 2007; Dingenmase et al. 2007), and it is clear that these personalities (shy or bold) (Gosling 2001) influence an individual's behavioral responses to a variety of environmental conditions (Sih et al. 2004a).

Studying behavioral flexibility with respect to behavioral syndromes is a more recent development. Behavioral syndromes describe suites of correlated behaviors reflecting between individual consistencies in behavior expressed within a behavioral 
context, also known as a situation (e.g., correlations between antipredator behaviors in different habitats), or across behavioral contexts (e.g., correlations among feeding, antipredator, or mating behavior) (Sih et al. 2004a). Basically, what an individual animal does in one behavioral situation can be correlated to what it does in another situation or context based on its behavioral type. A suite of correlated behaviors within a situation or across contexts is defined as a behavioral syndrome within a population when the rank order of the behavioral responses of individuals in the population is maintained within or across the context (Sih et al. 2004a; Sih et al. 2004b). Populations, species, and even groups of species can exhibit behavioral syndromes, with each individual or species showing a behavioral type within the larger syndrome (Sih et al. 2004a).

Individual behavioral repertoires may be constrained by their behavioral type within the larger behavioral syndrome (Sih et al. 2004a). For example, if the most aggressive, showy or bold individual in a low predation situation, is also the most aggressive, showy or bold individual in the high predation situation, that individual may express behavior that is not optimal (maladaptive) in the high predation situation. Also, traits involved in behavioral syndromes are coupled by underlying physiological mechanisms such as hormones; therefore, they must be looked at as a suite or whole system of related behaviors upon which natural selection may act (Sih et al. 2004a). In this study, I examined male guppy behaviors and interactions with other guppies including female courtship, competition for mates, and aggression on other males and whether or not behavioral syndromes within the same context such as aggression or courtship exist. 
Guppies are small, hardy, live-bearing, tropical poeciliid freshwater fish that inhabit still pools in clear, swift flowing streams, and the edges and backwaters of small rivers in mountain forest areas of Trinidad and Tobago, Venezuela, Guyana, and Suriname (Houde 1997; Magurran 2005). Guppies occur in stream habitats varying in a variety of environmental conditions, particularly predation pressure and light intensity (Houde 1997; Magurran 2005). Guppy populations described as high predation populations are subjected to major crustacean and fish predators, predominately the pike cichlid Crenicichla alta, while guppy populations described as low predation populations are subjected only to the minor gape-limited killifish, Rivulus hartii (Endler 1995; reviewed in Magurran 2005).

The behavior of guppies, especially male mating behavior, has been extensively described (Haskins and Haskins 1950; Baerands, Brouwer, and Waterbolk 1955; Liley 1966). Males utilize both an elaborate sigmoid courtship display to attract and mate with willing females, and sneaky copulations in which males attempt to sneak copulations without displaying to a female first (Houde 1997; Kelly and Godin 2001; Kolluru et al. 2007). Males also engage in aggressive interactions with other males. These interactions include both competitive interactions in which multiple males competitively court the same females, and dominance interactions in which males interact aggressively with each other without females nearby, potentially to establish relationships with respect to future mating rights to receptive females (Kolluru and Grether 2005; Kolluru et al. 2007).

Guppy streams vary in light intensity on a temporal and spatial scale due to variations in canopy cover, time of day, weather, water turbidity and water depth (Endler 1987; Luyten and Liley 1999; Reznick et al. 2001; Gamble et al. 2003; Kolluru et al. 
2007; Archard et al. 2009; Chapman et al. 2009). In guppy stream systems, the risk of detection by visually orienting predators increases with increasing light intensity. Male guppies become more conspicuous to $C$. alta at midday, when light intensity is greatest, than they are early and late in the day, and $C$. alta is more active at high light intensities (Endler 1987, 1991). Male guppies flexibly adjust their behavior under high predation risk conditions by performing fewer courtship displays and more sneak copulations (Endler 1987; Magurran and Seghers 1990; Magurran and Nowak 1991; Godin 1995; Houde 1997; Kolluru et al. 2007; Kelley and Brown 2011). Interestingly, males adjust their behavior in response to light levels in much the same way. Males perform fewer courtship displays under high (midday) light levels, perhaps in response to a perceived increased risk of predation given that $C$. alta is most active in high light (Reynolds 1993; Reynolds et al. 1993; Archard et al. 2009). Since males would seem to be the most conspicuous to choosy females under high light levels this seems to be a paradox (Endler 1987). Furthermore, Archard et al. (2009) showed that reduction in conspicuous courtship under high light conditions occurs independently of time of day. This evidence suggests that guppies directly perceive high light as being under high predation risk.

Broad arrays of animal taxa, including a wide variety of fish species, have been shown to display behavioral syndromes (Huntingford 1976; Drent et al. 2003; Reale and Festa-Bianchet 2003; Johnson and Sih 2005; see Conrad et al. 2011 for a review on fish). Studies have also investigated a wide range of potential behavioral syndromes including shy- bold continuums, and consistencies in exploration-avoidance, aggression, activity, and socialization (Conrad et al. 2011). Within fish, the majority of behavioral syndrome studies have been devoted to shy-bold personality consistency (Conrad et al. 2011). 
Studies on guppy fish have found individual behavioral consistencies in boldness and shyness, exploration and avoidance, and sociability (Budaev 1997; Croft et al. 2009; reviewed in Conrad et al. 2011). Furthermore, studies on guppy fish have indicated positive correlations between boldness and aggression, positive correlations between exploration, activity, and sociability, and a negative correlation between boldness and sociability (Budaev 1997; Croft et al. 2009; reviewed in Conrad et al. 2011). In this study, I used differences in light levels (high light and low light levels) to test individual male behavior across differing perceived predation risk situations to ascertain if behavioral syndromes for aggression and courtship exist in guppies.

\section{Methods}

Outline of Experimental Design: I examined the behavioral interactions between male guppies as a function of social environment (Female Present / Female Absent) in two light conditions (High Light/ Low Light) to ascertain how light level effects behavior to determine if a behavioral syndrome exists across light levels.

Study populations: The guppies used in this experiment were laboratory descendents of wild-caught fish collected from three geographically isolated streams representing 3 different drainage systems in Northern Trinidad: the Marianne River (referred to as MR fish) (PS 858 895), the Small Crayfish River (referred to as SC fish) (PS 965 835), and the Aripo River (referred to as AR fish) (PS 937 803) (Grether et al. 2001). These streams were chosen during surveys of stream drainages conducted in 1996 and 2000 (Grether et al. 2001) and were based on four criteria outlined in Grether et al. (2001): 1) intact old growth rainforest, 2) relatively uniform forest canopy cover, 3) geographic 
isolation from each other created by multiple barriers to guppy dispersal, 4) and low predation sites with no predatory fish except Rivulus hartii, a small, gape-limited predator (Grether et al. 2001; other details about these sites are given in Kolluru et al. 2007). Predation assemblage differences do exist between these sites such that the Marianne River contains diurnally active prawns (Macrobrachium crenulatum) (Millar et al. 2006; De Serrano et al. 2012) whereas the Aripo River (De Serrano et al. 2012) and the Small Crayfish River do not contain prawns, though the latter site may have contained prawns prior to the construction of the Hollis Dam in 1936 (http://wasa.gov.tt/WASA_Education_water_Reservoir_Hollis.html). All three sites are also likely to be subject to a variety of aerial predators such as birds (Templeton and Shriner 2004).

Laboratory setup: The Marianne River and Small Crayfish River populations were obtained from the laboratory of Gregory Grether at the University of California, Los Angeles in spring 2007. The Aripo River population was obtained from Brian Smith in the laboratory of Dan Blumstein at the University of California, Los Angeles in fall 2008. All fish were transported to the Kolluru laboratory at California Polytechnic State University, San Luis Obispo, California, via automobile, in aerated, temperaturecontrolled containers.

Fish stocks were maintained in multiple mixed-sex 10-gallon stock tanks to allow for breeding and to minimize inbreeding. These tanks contained natural, multi-colored gravel, Java moss (Taxiphyllum barbieri), to provide areas to hide in, and trumpet snails (Melanoides tuberculata) to help maintain appropriate water conditions. The lab temperature was maintained at $25 \pm 0.5^{\circ} \mathrm{C}$ and the fish were exposed to $12: 12 \mathrm{~L}$ : D cycle 
using full spectrum fluorescent (Philips Home Light Natural Sunlight Full-Spectrum Light; 2950 lumens; 32 watt) and LED light sources. The stock populations were fed TetraMin ${ }^{\circledR}$ Tropical flakes (Tetra Holding, Inc.) twice per day during the week and once per day on the weekends, periodically supplemented with Ocean Star® International Spirulina flakes and Hikari® frozen brine shrimp.

In April of 2008, 20 healthy females from each population were isolated in individual 2-gallon plastic tanks with a healthy male from the same site, and allowed to give birth. Because the females were chosen from stock populations, they were most likely gravid by the time they were isolated, however, the companion male in each 2gallon tank may have fathered some of the offspring as well. These tanks were outfitted with plastic nets that divided the tank, allowing fry to swim away from potentially cannibalistic adults. Each tank contained gravel, and Java moss for cover. These tanks experienced the same conditions and feeding schedule described above.

Offspring were removed from the female's tank at 1-3 weeks of age, and transferred to 2-gallon tanks containing multi-colored gravel and moss, at densities of 2 to 6 fish per tank, with each tank containing representatives from no more than two litters with some tanks containing only single litters. At approximately ten weeks of age, the juveniles were sexed and separated into single-sex 2-gallon tanks. Sex was determined by the presence of pigmentation and gonopodium development in males, and dark coloration around the anal fin in females (Houde 1997). After sexing, two types of single-sex 2gallon tanks (hereafter referred to as "Home Tanks") were set up: male tanks contained 1 to 4 males, and female tanks contained 1 to 3 females. Because offspring were sexed and separated before completion of development of the gonopodium in males, I am confident 
that the fish were all virgins prior to observations (Houde 1997). After they were sexed and separated, males and females did not have any visible contact with each other, to minimize the influence of visual contact between the sexes on male competitive interactions and female choice (Grether 2000; Hibler and Houde 2006).

Focal Behavioral Observations: Observations occurred between March and December 2009. Males were assigned, randomly constrained by size match, to one of two social environment treatments (Females Present or Females Absent). Female Present social treatments consisted of two female and two male fish in the observation tank together during the trial period while Female Absent social treatments consisted of just two male fish together in the observation tanks during the trial period. Each male was observed under both Low Light and High Light levels (on subsequent days, in a randomized order) but under only one of the two social environment treatments (Females Present or Females Absent). I also randomized the order of testing by population (MR, AR or SC), light treatment on Day 1 (High Light or Low Light), and which of two identically outfitted observation tanks would be used for the observations (tank 1 or tank 2; see below for description of observation tanks) such that all possible combinations were equally represented. Individual males to be observed were chosen based on the following criteria: 1) the oldest available males; 2) males from tanks containing more than 1 male; 3) visibly healthy males; 4) sized matched males (determined by observing body length with the naked eye); 5) males that were born within three weeks of each other; and 6) males whose home tanks were on different shelves in the laboratory, further minimizing the chances of prior visual contact. The chosen males' color patterns were sketched to enable individual identification. 
The two focal males chosen on a given day were paired with each other for the first time for the behavioral trial. Behavioral observations were conducted in one of two 20-gallon observation tanks ( $76.2 \times 31.75 \times 31.7512 .5$ centimeters $)$ containing multicolored gravel bottoms and plastic bubblers connected to under gravel filters (Figures 2 and 3). The back and sides of each tank were covered in a uniform brown paper background. The temperature in the tanks was maintained at the laboratory temperature of $25^{\circ} \pm 5^{\circ} \mathrm{C}$ and the tanks were filtered between trials with charcoal canister filters (Marineland® H.O.T. Magnum 250 HSB Canister Filter) to minimize chemical effects on the behavior of fish in subsequent trials (Crow and Liley 1979). The observation tank area was blocked off from the rest of the laboratory (including the general room lighting) by a heavy black curtain that extended completely around the tanks. Two sets of fullspectrum florescent lights (Vita-Lite $® ; 30$ watt; Dura-test 07-15121) and several sets of LED lights spanned the length of the two observation tanks. The lights were mounted 22.86 centimeters above each tank, and each set of lights included two full spectrum light tubes that stretched 91.44 centimeters across, providing even illumination over the two tanks. Small strips of LED lights were placed between each set of fluorescent lights. Fish were fed TetraMin® Tropical Flake food to satiation 15 minutes before the first observation period and immediately after the second observation period, to minimize competition for food (Magurran and Seghers 1991).

Photosynthetically active radiation levels were measured using a $\mathrm{Li}-250 \mathrm{~A}$ Quantum/Radiometer/ Photometer light meter (Li-Cor Biosciences Inc.) equipped with a Li-190SA quantum sensor, and all readings were taken in the middle of each tank and approximately 2.54 centimeters above the water surface. The Low Light level was 
defined as 5 to $25 \mu \mathrm{mol} / \mathrm{m}^{2} / \mathrm{s}$ and the High Light level as 70 to $90 \mu \mathrm{mol} / \mathrm{m}^{2} / \mathrm{s}$. These light levels were based on a behaviorally active range of light values determined by field measurements of photosynthetically active light levels above Trinidadian guppy streams (Grether et al. 2001; Kolluru and Grether 2004; Kolluru et al. 2007), and on previous laboratory studies of behavioral plasticity across light levels (Reynolds et al. 1993), as well as the lowest light levels under which behaviors could be seen. High Light was achieved by turning on all observation tank lights, and Low Light conditions were achieved by turning on all observation tank lights and placing a double layer of black shade cloth over the top of the observation tanks, thereby reducing light intensity at the water level (see Gamble et al. 2003 for a similar use of shade cloth).

A trial was initiated by netting the males from their home tanks, setting the proper light conditions for Day 1 over both tanks, allowing the two males to acclimate to observation tank water conditions for 5 minutes in two separate clear caddies in which they could see each other, and releasing the two males together into one of the two observation tanks, chosen at random, between 900 and 1100 (PST) hours. If the social treatment was "Females Present", two size-matched, virgin females from two different home tanks and from the same site as the males were chosen. The females were acclimated to water conditions for 5 minutes in separate caddies and released into the observation tank with the males. After releasing the fish, I fed them a small pinch of flake food as described above, and closed the curtain, visually isolating the observation tanks from the rest of the lab.

Observation sessions began between 1500 and 2000 (PST) hours, on the same day the fish were introduced into the observation tanks. The fish were fed approximately 15 
minutes prior to observations. I performed 3 five-minute focal male observations per male with a minimum of 15 minutes between consecutive focal observations on a given male. Males were observed alternately, in a randomly chosen order. The fish were fed again after the second focal observation to avoid food competition. After the observations were complete on Day 1the lights were turned off and the curtain closed around the observation tank area.

The second day of observation ("Day 2") began between 900 and 1100 hours (PST). The light levels were changed to the opposite of what they were on Day 1 and the fish were fed. Observations for Day 2 occurred between 1500 and 2000 hours (PST) and followed the same pattern described above for Day 1. After the conclusion of observations on Day 2 the fish were anesthetized using MS 222 (tricaine methanesulfonate; 200mg/L of water; Finquel; Argent Chemical Laboratories), weighed to the nearest $0.1 \mathrm{mg}$, their standard length was measured to the nearest $0.01 \mathrm{~mm}$ using digital calipers, and digital images of the left and right lateral surfaces were taken for future analyses of color patterns. Males were allowed to recover in fresh water. After sufficiently recovered, males were returned to stock tanks to contribute to the laboratory stocks. All data were recorded on a Macintosh Power Book G4 computer (OSX operating system; Apple) using a True Basic Silver Edition behavior event-recording program written by J.C. Walz, based on a program written by G.F. Grether.

I conducted 48 trials, involving a total of 96 males ( $\mathrm{n}=$ Marianne River, 32; Small Crayfish River, 32; Aripo River, 32). Half of the trials (24 trials) involved the social treatment "Females Absent" ( $\mathrm{n}=$ Marianne River, 16; Small Crayfish River, 16; Aripo 
River, 16) and the other half, with equal sample sizes of males and trials, involved the "Females Present" social environment treatment.

Behavioral Variables: Behaviors recorded were those collected by Kolluru and Grether (2005), with the addition of the lateral display and face-offs (Table 1). The following variables were recorded for each male: follow, nip, sigmoids, competition, sneaks, swings, switch, display, competition/dominance, chase, scuffle, bite, lateral display, foraging, face-off and moving. In focal observations in which females were absent the following behaviors were excluded since they are exclusive to male behavior towards females: courtship display, competition, competition/dominance, follow, and nip.

Data Analysis: All behaviors were computed as rates (per 900 seconds of observation), with the exception of chases, for which I computed proportion of time spent chasing (total chase duration per 900 seconds of observation). Chases were computed this way due to the fact that a chase was recorded not as a discrete event but rather a record over time of how long a chase lasted. Competition rate was analyzed using composite variables of scored competitive events that reflected the rates that males instigated a competition with another male. All data analyses were performed using JMP Pro 10.0.1 software (SAS® Institute, Inc. 2012).

A Principal Components Analysis (PCA) was used to collapse the potentially correlated behavioral measures into component axes describing behavior. I performed separate PCAs for the two social environments (Female Present and Female Absent) and two light situations (High Light and Low Light). Only components that resulted in eigenvalues above 1.0 were retained. All loadings from the behavioral components 
described by the PCA for behavior in the two light conditions and the two social environments were used to calculate the principal component scores for male behavior in the two social environments and across both light situations. The retained component scores were used in subsequent Spearman's rank correlation analyses to determine if any correlations (i.e., behavioral syndromes) existed across light treatments. The Spearman's rank correlation analysis was performed separately for each social condition. A global Bonferroni correction was used to correct for multiple tests. In addition, Spearman's rank analyses were performed on the retained significant component scores at the population level to determine which populations expressed and/or were driving behavioral syndromes between light levels. For a similar analyses of behavioral syndromes see Wilson and Godin 2009.

\section{Results}

\section{Principal Components Analysis (PCA) Results}

A similar component in both High and Low light conditions emerged in the PCA's of the Female Absent social treatment group (Table 2 and 3). This component described "male-male aggression", with high positive loadings for aggressive behavior, including chases and bites, and high negative loadings for foraging (Tables 2 and 3). A large positive component score resulted when a male chased and bit other males frequently, compared to a male that expressed less aggressive behavior towards other males or spent more time foraging, which resulted in a smaller positive and /or negative component score.

The PCA of behavior in Female Present social situations resulted in three components in both high and low light situations (Tables 4 and 5). The first component 
described a "courtship" axis, with high courtship behaviors and low male-male aggression loadings, in both high and low light conditions. Males who courted a lot had relatively larger scores on this component than those males who engaged in aggression towards other males; the latter may have resulted in negative component scores.

Component two in both high and low light conditions described aggressive behaviors directed at other males, including chases, bites and interference competition for a female, and was characterized as an "aggression" axis (Tables 4 and 5). Males with larger positive component scores were more aggressive and competitive with other males.

Component three in both high and low light situations involve aggressive and courtship behavior versus engaging in sneak copulation. However, the pattern of loadings differed between low and high light situations (Table 4 and 5). Due to the differences in this component across light levels, we excluded it from further analysis.

\section{Spearman's Rank Correlations}

Spearman's rank correlations were used to determine the ranked relationships between the PCA component scores of male behavior in High and Low light situations. A Spearman's rank coefficient of +1 would describe a strong positive relationship between the ranking of a males PCA component score in low light and high light, while a coefficient of -1 would describe a strong negative relationship. As the coefficient approaches 0 there is not a strong relationship between the two rankings. I performed three Spearman's rank correlations, between High and Low light, for each of the following combinations: aggression in Female Absent social situations, 
aggression/competition in Female Present situations, and courtship in Female Present situations.

In the Female Absent treatment, there is a behavioral syndrome for the only significant component, aggression, which was significantly correlated across light situations ( $\rho=0.51, P=0.0002$; Table 6$)$. The positive Spearman's rank coefficient indicates that the guppies show higher levels of inter- male aggression in low light than in high light, but maintained their rank order relationships in how aggressive they were (Figure 4). Therefore, males that are aggressive in high light appear to be aggressive in low light as well (Figure 4).

For behavior in Female Present situations there was a behavioral syndrome described by all behavioral components, all of which were significantly correlated across the High and Low light situations. For component one, the courtship axis, the Spearman's rank correlation was significant $(\rho=0.56, \mathrm{P}<0.0001$; Table 7$)$. As the positive Spearman's rank coefficient indicates, males exhibited a behavioral syndrome, individually courting as much under low light situations as they were courting under high light situations and being more aggressive towards other males in low light situations (Figure 5). Component two describing aggressive and competitive behavior was significant as well $(\rho=0.35, P=0.016$; Table 7$)$. Again, the positive coefficient suggests a positive slope from high to low light in male directed aggression and competition (Figure 5). All Spearman's rank correlation coefficients remained significant after a Bonferroni correction for multiple tests within the Female Present social condition ( $\alpha$ corrected $=0.0166$ ) 
To determine whether particular populations were driving the syndromes, I performed the correlation analyses separately by population. Among Marianne River and Small Crayfish populations the aggression component in Female Absent social conditions exhibited a syndrome across light conditions but there was no syndrome within the Aripo River population (Aripo $\rho=0.4441, \mathrm{P}=0.0848$; Marianne $\rho=0.5088, \mathrm{P}=0.0441$; Small Crayfish $\rho=0.5676, P=0.0218$; Table 8 ; Figure 7). After Bonferroni correction $(\alpha$ corrected $=0.0166$, however, no populations displayed any significant correlations across light conditions.

The Aripo River and Marianne River populations, but not Small Crayfish populations, exhibited a syndrome for the courtship component in Female Present social environments across light conditions (Aripo $\rho=0.5765, P=0.0194$; Marianne $\rho=0.6412$, $\mathrm{P}=0.0074$; Small Crayfish $\rho=0.1265, \mathrm{P}=0.6407$; Table 9; Figure 8). After Bonferroni correction ( $\alpha=0.0166)$ only Marianne River populations exhibited a syndrome for courtship across light conditions. Only Aripo River populations show a significant correlation across light conditions for the aggression component even after Bonferroni correction (Aripo $\rho=0.7029, \mathrm{P}=0.0024$; Marianne $\rho=0.1765, \mathrm{P}=0.5133$; Small Crayfish $\rho=0.2588, P=0.3331$; Table 10; Figure 9).

\section{Discussion}

I found evidence for behavioral syndromes for aggression and courtship across light levels in both the presence and absence of sexually receptive females. The rank order of males with respect to these behaviors was therefore preserved. No single population drove the aggressive behavioral syndrome when there were no sexually 
receptive females. When females were present, only Marianne River fish displayed a syndrome for courtship and only Aripo River fish exhibited a syndrome for aggression/competition. The other populations showed no rank order in their behavioral flexibility between light conditions, suggesting behavioral flexibility between light environments.

Behavioral syndromes have been observed in guppies and other poeciliid species, typically involving boldness in approaching a predator or exploring a novel environment, as well as aggression (Budaev 1997; Reisch et al. 2009; Harris et al. 2010; See Conrad et al. 2011 for review). A study with the western mosquito fish (Gambusia affinis) found behavioral syndromes across boldness, exploratory behavior, sociability, and activity (Cote et al. 2010). In bishop livebearers (Brachyrhaphis episcopi), Brown et al. (2007) found positive behavioral correlations among boldness and exploratory behavior. Several studies involving guppies have found behavioral syndromes across boldness and aggression, and across exploration, activity, and sociability (Budeav 1997; Croft et al. 2009; Piyapong et al. 2010). Many other freshwater fish (e.g., stickleback (Gasterosteus aculeatus), sunfish (Lepomis macrochirus), zebrafish (Danio rerio), as well as broad spectrum of other taxa, have also shown similar behavioral syndromes, particularly across contexts such as boldness, exploration, activity, and aggressiveness (reviewed in Conrad et al. 2010).

The existence of a syndrome would suggest that behavior across an environmental gradient cannot be optimally regulated by the male based solely on the environmental situation faced, but is rather reflected in that male's particular behavioral type. I show that males maintain their rank order levels of aggression and courtship with changes in 
light situations (predation risk) and have an established behavioral syndrome across light situations for these behaviors in both female absent and female present social situations. The most conspicuous males in low light and potentially less risky environments are also the most conspicuous males in high light and potentially more risky environments. There may nonetheless be mean changes across light levels such that there is adaptive plasticity despite the syndromes.

Predators may prey more on unwary individuals (FitzGibbon1989; Krause and Godin 1996). Males that are aggressive and courting females are also less likely to be aware of their potential risk (Magurran and Seghers 1994; Cooper and Federick 2007). However, being conspicuous may have direct mating benefits. A male who consistently courts females, despite the risks, may potentially increase his chances of mating, and this benefit may outweigh the risk of predation.

In both the presence and absence of receptive females, a clear syndrome for aggressiveness to other males emerged. Male dominance has been shown to enhance mating success in guppies (Kodric-Brown 1992). It may be important to males to maintain courtship and aggressiveness because the more a male displays the more mating opportunities he may have. Kodric -Brown (1993) determined that male mating success was correlated with conspecific agonistic dominance behavior, intensity of the male courtship display and male color patterns. Furthermore, high rates of display were attractive to females, and a female's visual response to dominance behaviors is a good indicator of the male's mating success (Kodric- Brown 1993; Kodric-Brown and Nicoletto 2001). However, the relationship between aggression and reproductive success 
is unclear as a variety of studies with differing populations have found conflicting results (Gandolfi 1971; Gorlick 1976; Houde 1988; Kolluru and Grether 2005)

In this study the behavioral syndromes for male aggression and courtship across light situations are not expressed in all three of our populations. When females are absent no single population drove the overall syndrome. However, when there are sexually receptive females, Marianne River and Aripo River show strong correlations in courtship behaviors and aggression across light situations, respectively. Bell (2005) found a similar situation in which only one of two studied populations of stickleback fish, Gasterosteus aculeatus, from two separate drainages in California exhibited a behavioral syndrome. These fish exhibited syndromes across contexts of boldness towards a predator, aggression towards conspecifics, and activity in an unfamiliar environment. Bell (2005) suggests that differences in predation pressure or regime may account for differences in whether or not a behavioral syndrome may exist within a population. Within Bell's (2005) two populations only the one with greater predation pressure exhibited a behavioral syndrome.

Predation pressure has been suggested to be an important selective mechanism in generating behavioral syndromes (Smith and Blumstein 2010). Investigation into predation risk and behavioral syndromes in sticklebacks suggest that populations that experience significant predation pressure express a syndrome for boldness and aggression, which is not reflected in populations that experience reduced or no predation (Bell and Stamps 2004; Bell 2005; Dingemanse et al. 2007; Dingemanse et al. 2010). Interestingly, Bell and Sih (2007) found that even mere exposure to a predator can generate a boldness-aggression syndrome. All our guppies were lab descendants of low 
predation populations and were reared in a predator-free environment. Low predation populations of guppies are still prone to predators such as the killifish Rivulus hartii, but have adapted to environments not subjected to the voracious predator C. alta (Liley and Seghers 1975; Endler 1987; Reynolds 1993). These populations do express behavioral syndromes for both aggressive behavior and courtship behavior across light situations, and as such do not mediate their conspicuousness across an environmental gradient that could increase their potential predation risk (Haskins et al. 1961; Endler 1987). My findings would warrant further research into determining if low predation populations of guppies act in similar ways to sticklebacks such that boldness in high predation situations by guppies is not impacted by selection favoring low aggressiveness (Sih et al. 2012).

Syndromes themselves might be advantageous, even in risky high predation/ high light environments. Smith and Blumstein (2010), utilizing the work of Lopez et al. (2005), postulate that if boldness is condition-dependent than an individual's consistency of position on the boldness scale may reflect its ability to evade predators. In a similar manner, it is possible that the consistency of position of a guppy on the aggression or courtship scale in our study may reflect an honest signal of a guppy's likelihood of winning a fight or its ability to maintain courtship, a behavior that requires plenty of energy. Guppy carotenoid based colors act as an honest signal of male quality because carotenoid pigments must be obtained from the diet, and are limited in the wild (Goodwin 1984; Grether et al. 1999). Several studies have shown that male guppies with brighter orange carotenoid based spots have better foraging ability, are healthier fish, may carry more disease resistance genes and are likely to be more resistant to parasite infection (Endler 1978; Hamilton and Zuk 1982; Folstad and Karter 1992; Houde and Torio 1992, 
Grether et al. 2004; Magurran 2005). Furthermore, males with more carotenoid based color spots have also been shown to have better predator escape behaviors (Godin and Dugatkin 1996) and offspring fathered by males with more carotenoid based colors were more adept at avoiding predation (Evans et al. 2004). Although all three of our populations are lab descendants of low predation populations, there exists the possibility that a male's consistency of position on the continuum of aggression and courtship (conspicuousness) between light situations reflects an honest indicator to females about a male's ability to evade predation and about his individual health, and that those males on the high end of conspicuousness can afford to always be more conspicuous. Some studies suggest that selection for conspicuous color patterns and high display rates is more intense in populations under low predation pressure (Luyten \& Liley 1991; Endler \& Houde 1995; Kodric-Brown 1999). Energetically costly behaviors, such as high display rates, may be favored by selection because these behaviors honestly advertise a male's physical condition (Kodric-Brown \& Brown1984; Kodric-Brown 1989). Females that choose males that display more across light situations would obtain mates that are, on average, in better physical condition.

How individual animals deal with changes in light level in their natural environment is an important area of study, especially with increasing human induced changes to our natural world, such as deforestation. Endler (1995) noted that more light can lead to a chain reaction of changes in guppy behavior and life history strategies, including, but not exclusively limited to, increasing the food supply, which increases the availability of carotenoids, leading to less sexual selection, influencing the blending of color patterns at distances, leading to more dull coloration; and creating an environment 
suited to a more 'r-selected' life history strategy (Endler 1995; Magurran 2005).

Behavioral modifications are often a first response to environmental changes, including human induced alterations. Behavioral syndromes that result in differential fitness effects of an individual's behavioral type have ecological and evolutionary importance, and thus the study of relevant ecological factors such as predation and light changes is a relevant and important area of study (Sih et al. 2012). 


\section{Tables:}

Table 1: Description of the male behaviors recorded in the Social Challenge focals.

\begin{tabular}{|c|c|}
\hline Behavior & Description \\
\hline follow & $\begin{array}{l}\text { Male follows one or both of the females } \\
\text { around the tank }\end{array}$ \\
\hline nip & Male nips/bites at one of the females \\
\hline courtship display (sigmoid) & $\begin{array}{l}\text { Male turns his body into an s shape in front } \\
\text { of the female and begins to quiver }\end{array}$ \\
\hline competition & $\begin{array}{l}\text { Male is following a female and the other } \\
\text { male begins to follow the same female (in } \\
\text { competitions males can sigmoid to females, } \\
\text { nip females, etc...) }\end{array}$ \\
\hline sneak copulation & $\begin{array}{l}\text { Male, tries to copulate with a female } \\
\text { without courting a female using the } \\
\text { sigmoid display }\end{array}$ \\
\hline gonopodial swings & $\begin{array}{l}\text { Male moves his gondopodium in an } \\
\text { upward arc }\end{array}$ \\
\hline switch & $\begin{array}{l}\text { Male is following one female and then } \\
\text { switches females and begins to follow } \\
\text { another female }\end{array}$ \\
\hline display & $\begin{array}{l}\text { One male performs a display clearly } \\
\text { directed at the other male; this display } \\
\text { closely resembles the characteristic } \\
\text { "sigmoid" courtship display of guppies }\end{array}$ \\
\hline competition/dominance & $\begin{array}{l}\text { The two males are engaged in a } \\
\text { competition but leave the females and } \\
\text { begin to do male only behaviors such as } \\
\text { chase, display, bite. }\end{array}$ \\
\hline chase & $\begin{array}{l}\text { One male swims directly towards the other } \\
\text { male at a rapid pace }\end{array}$ \\
\hline scuffle & $\begin{array}{l}\text { The two males repeatedly chase and bite } \\
\text { each other, but it is not obviously instigated } \\
\text { by a particular male }\end{array}$ \\
\hline bite & $\begin{array}{l}\text { One male nips at/bites the other male } \\
\text { without chasing }\end{array}$ \\
\hline
\end{tabular}




\begin{tabular}{|l|l|}
\hline lateral display & $\begin{array}{l}\text { The two males are parallel to each other } \\
\text { with fins splayed and begin to exhibit a } \\
\text { vibrating motion }\end{array}$ \\
\hline foraging & Male is feeding \\
\hline face-off & $\begin{array}{l}\text { The two males face each other and hover or } \\
\text { swim in a parallel line }\end{array}$ \\
\hline $\begin{array}{l}\text { A male is not following a female, engaging } \\
\text { in activities with the other male, and/or not } \\
\text { feeding. Often characterized by swimming } \\
\text { up and down against the glass of the tank. }\end{array}$ \\
\hline
\end{tabular}


Table 2: Results of principal component analysis on behavioral measures recorded in Female Absent trials in High Light situations. Component loadings that are bold are significant loadings $>0.4$.

\begin{tabular}{|lc|}
\hline & $\begin{array}{c}\text { Male }- \text { Male Aggression } \\
\text { Component }\end{array}$ \\
\hline Eigenvalues & 1.9840 \\
\% variance explained & $49.6 \%$ \\
Chase duration & \\
Display totals/s & $\mathbf{0 . 8 6 2 6 7}$ \\
Forage totals/s & $\mathbf{0 . 5 6 2 8 0}$ \\
Bite totals/s & $\mathbf{- 0 . 4 5 7 6 3}$ \\
\end{tabular}

Table 3: Results of principal component analysis on behavioral measures recorded in female absent trials in Low Light situations. Component loadings that are bold are significant loadings $>0.4$.

\begin{tabular}{|lc|}
\hline & $\begin{array}{c}\text { Male }- \text { Male Aggression } \\
\text { Component }\end{array}$ \\
\hline Eigenvalues & 2.1603 \\
& $54.0 \%$ \\
Behariance explained & \\
Chase duration & $\mathbf{0 . 7 7 7 5 9}$ \\
Display totals/s & $\mathbf{0 . 7 8 4 7 3}$ \\
Forage totals/s & $\mathbf{- 0 . 4 8 0 0 9}$ \\
Bite totals/s & $\mathbf{0 . 8 4 2 2 5}$ \\
\hline
\end{tabular}


Table 4: Results of principal component analysis on behavioral measures recorded in female present trials in high light situations. Component loadings that are bold are significant loadings $>0.4$.

\begin{tabular}{|c|c|c|c|}
\hline & $\begin{array}{c}\text { Courtship } \\
\text { Component }\end{array}$ & $\begin{array}{l}\text { Aggression/ } \\
\text { Competition } \\
\text { Component }\end{array}$ & $\begin{array}{c}\text { Courtship/ } \\
\text { Competition } \\
\text { versus Sneak } \\
\text { Component }\end{array}$ \\
\hline Eigenvalues & 2.0786 & 1.5405 & 1.3019 \\
\hline$\%$ variance explained & $26.0 \%$ & $19.3 \%$ & $16.3 \%$ \\
\hline \multicolumn{4}{|l|}{ Behaviors } \\
\hline Chase duration & -0.37788 & 0.74858 & -0.19840 \\
\hline $\begin{array}{l}\text { Male/Male Display } \\
\text { totals/s }\end{array}$ & 0.07872 & 0.22201 & -0.58520 \\
\hline Bite totals/s & -0.54485 & $\mathbf{0 . 5 5 5 3 7}$ & -0.19700 \\
\hline Forage totals/s & -0.42702 & -0.33662 & 0.37420 \\
\hline Nip totals/s & 0.69856 & -0.07174 & -0.21826 \\
\hline Competition Instigations/s & 0.49682 & 0.43697 & 0.52902 \\
\hline Courtship totals/s & 0.82591 & 0.19528 & -0.23759 \\
\hline Attempted Sneak totals/s & 0.18307 & 0.52431 & 0.59771 \\
\hline
\end{tabular}


Table 5: Results of principal component analysis on behavioral measures recorded in female present trials in low light situations. Component loadings that are bold are significant loadings $>0.4$.

\begin{tabular}{|c|c|c|c|}
\hline & $\begin{array}{c}\text { Courtship } \\
\text { Component }\end{array}$ & $\begin{array}{l}\text { Aggression/ } \\
\text { Competition } \\
\text { Component }\end{array}$ & $\begin{array}{c}\text { Courtship/ } \\
\text { Competition } \\
\text { versus Sneak } \\
\text { Component }\end{array}$ \\
\hline Eigenvalues & 2.1272 & 1.6381 & 1.3914 \\
\hline$\%$ variance explained & $26.6 \%$ & $20.5 \%$ & $17.4 \%$ \\
\hline \multicolumn{4}{|l|}{ Behaviors } \\
\hline Chase duration & -0.51576 & 0.65555 & -0.00139 \\
\hline $\begin{array}{l}\text { Male/Male Display } \\
\text { totals/s }\end{array}$ & -0.29602 & -0.11926 & 0.69411 \\
\hline Bite totals/s & -0.64273 & 0.58885 & 0.17012 \\
\hline Forage totals/s & -0.38553 & -0.62373 & -0.45209 \\
\hline Nip totals/s & 0.72942 & 0.25020 & 0.12269 \\
\hline Competition Instigations/s & 0.25538 & 0.56168 & -0.31358 \\
\hline Courtship totals/s & 0.72759 & 0.04574 & 0.42671 \\
\hline Attempted Sneak totals/s & 0.29181 & 0.27960 & -0.61713 \\
\hline
\end{tabular}


Table 6: Spearman's Rank correlation data for PCA results for male guppy behavior in Female Absent trials between High light and Low Light Situations. The * indicates statistically significant correlations.

\begin{tabular}{|ccc|}
\hline Behavior & Spearman's Rank $\rho$ & $P$ \\
\hline & & \\
Aggression & 0.5144 & $0.0002^{*}$ \\
& & \\
\hline
\end{tabular}

Table 7: Spearman's Rank correlation data for PCA results for male guppy behavior in Female Present trials between High light and Low Light Situations. The * indicates statistically significant correlations. Bonferroni adjusted significance for 3 tests is alpha at 0.0166 .

\begin{tabular}{|ccc|}
\hline Behavior & Spearman's Rank $\rho$ & $P$ \\
\hline Courtship & 0.5599 & $<.0001^{*}$ \\
Aggression/Competition & 0.3461 & $0.0160^{*}$ \\
\hline
\end{tabular}


Table 8: Spearman's rank results for female absent trials by population (AR: Aripo; MR: Marianne; SC: Small Crayfish) results. There was no significant population results after a strict Bonferroni correction $(\alpha=0.0166)$.

\begin{tabular}{|ccc|}
\hline Behavior & Spearman's Rank $\rho$ & $P$ \\
\hline $\begin{array}{c}\text { Male-Male } \\
\text { Aggression }\end{array}$ & 0.5144 & $0.0002^{*}$ \\
AR & 0.4441 & 0.0848 \\
MR & 0.5088 & 0.0441 \\
SC & 0.5676 & 0.0218 \\
\hline
\end{tabular}

Table 9: Spearman's rank results for female present courtship component by population (AR: Aripo; MR: Marianne; SC: Small Crayfish). The MR population results after a strict Bonferroni correction $(\alpha=0.0166)$ were significant.

\begin{tabular}{|ccc|}
\hline Behavior & Spearman's Rank $\rho$ & $P$ \\
\hline Courtship & 0.5599 & $<0.0001^{*}$ \\
AR & 0.5765 & 0.0194 \\
MR & 0.6412 & $0.0074^{*}$ \\
SC & 0.1265 & 0.6407 \\
\hline
\end{tabular}


Table 10: Spearman's rank results for female present aggression component by population (AR: Aripo; MR: Marianne; SC: Small Crayfish). The Aripo population results after a strict Bonferroni correction $(\alpha=0.0166)$ are significant.

\begin{tabular}{|ccc|}
\hline Behavior & Spearman's Rank $\rho$ & $P$ \\
\hline Aggression/Competition & 0.3461 & $<0.0160^{*}$ \\
AR & 0.7029 & $0.0024^{*}$ \\
MR & 0.1765 & 0.5133 \\
SC & 0.2588 & 0.3331 \\
\hline
\end{tabular}




\section{Figures:}

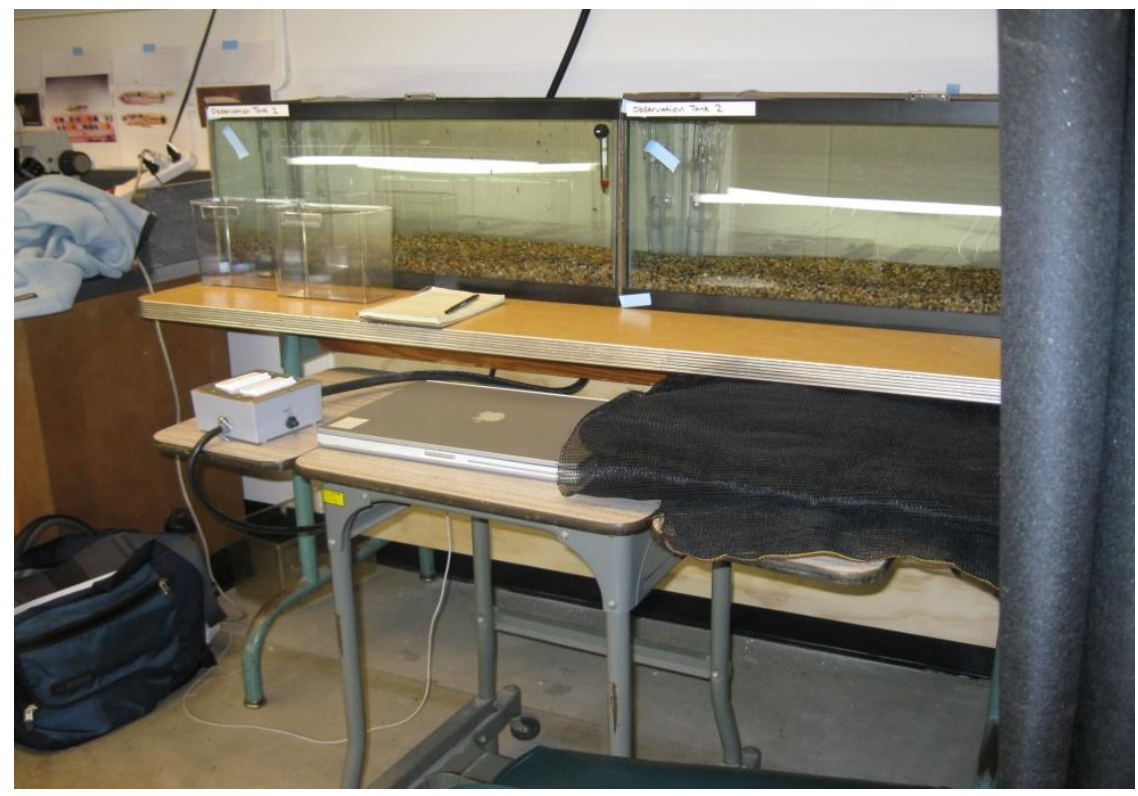

Figure 2: The set up of the two observation tanks. Fish were placed in one of the two identical tanks pictured. The small caddies seen in front of Tank 1 (the observation tank on the left) were used as acclimation chambers. The small table in front held the computer used for recording behaviors. To the right of the computer is the piece of shade cloth used to create low light levels on the tank. The piece was laid across the top of two tanks, effectively blocking the light created from the light sources above the tanks (not seen in this image). The black curtain to the far right could be pulled around to separate the observation tanks from the rest of the room. 


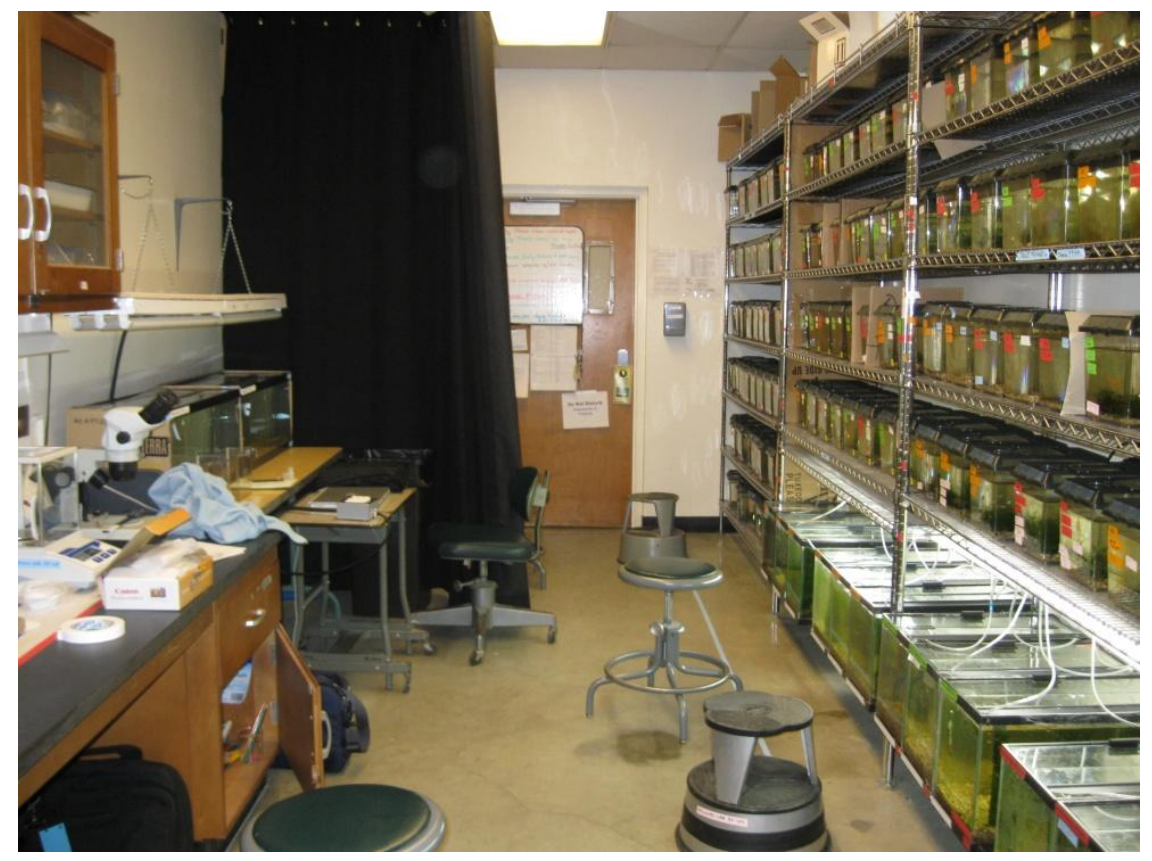

Figure 3: The lab room at Cal Poly, San Luis Obispo. Home Tanks are on the racks on the right side of the image and the observation tanks are on the left side of the image, near the black curtain. 


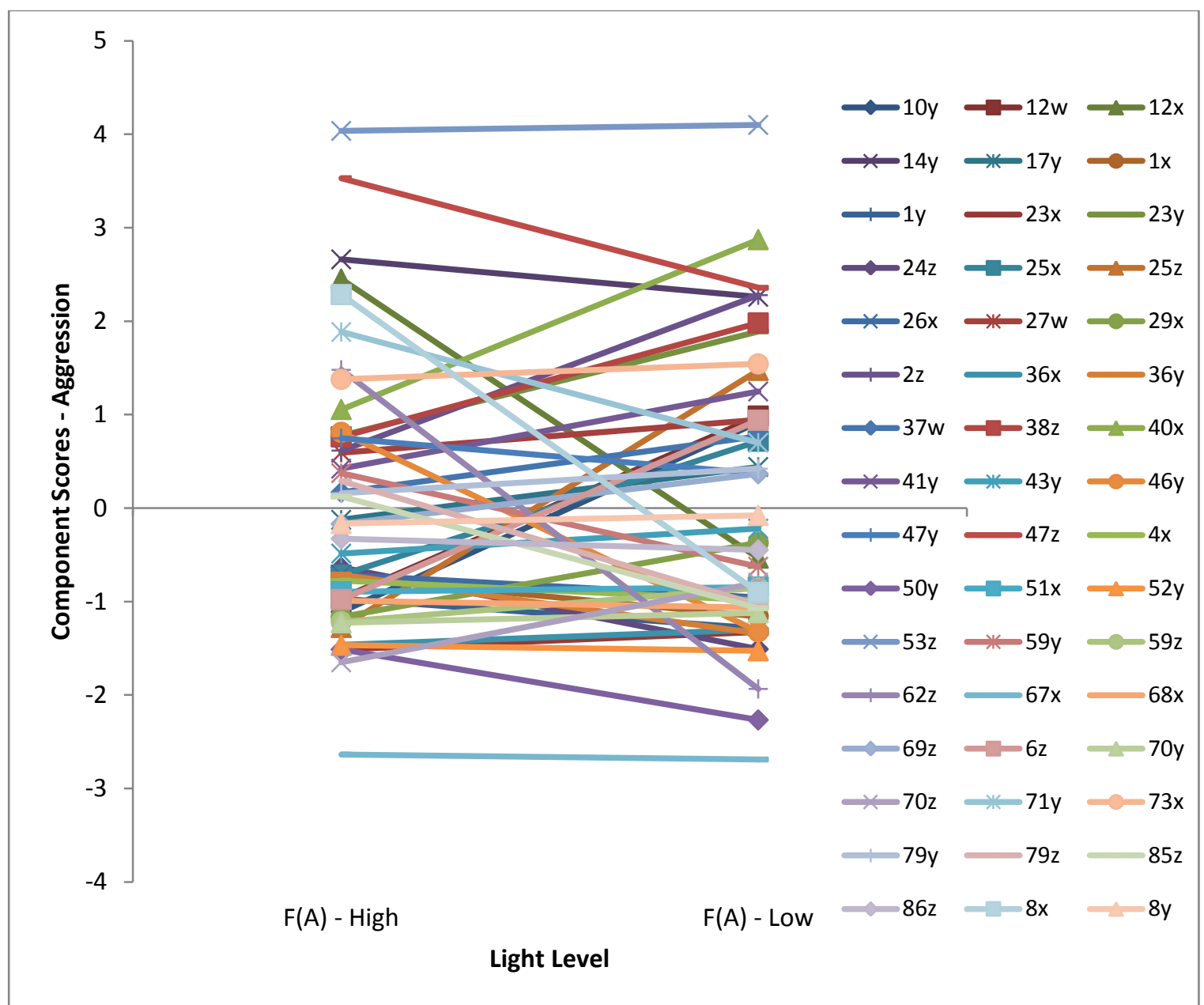

Figure 4: Reaction norm plot for female absent aggression component between high and low light levels. Each male's component score in high and low light is represented by a particular line with the legend showing Male ID numbers. 


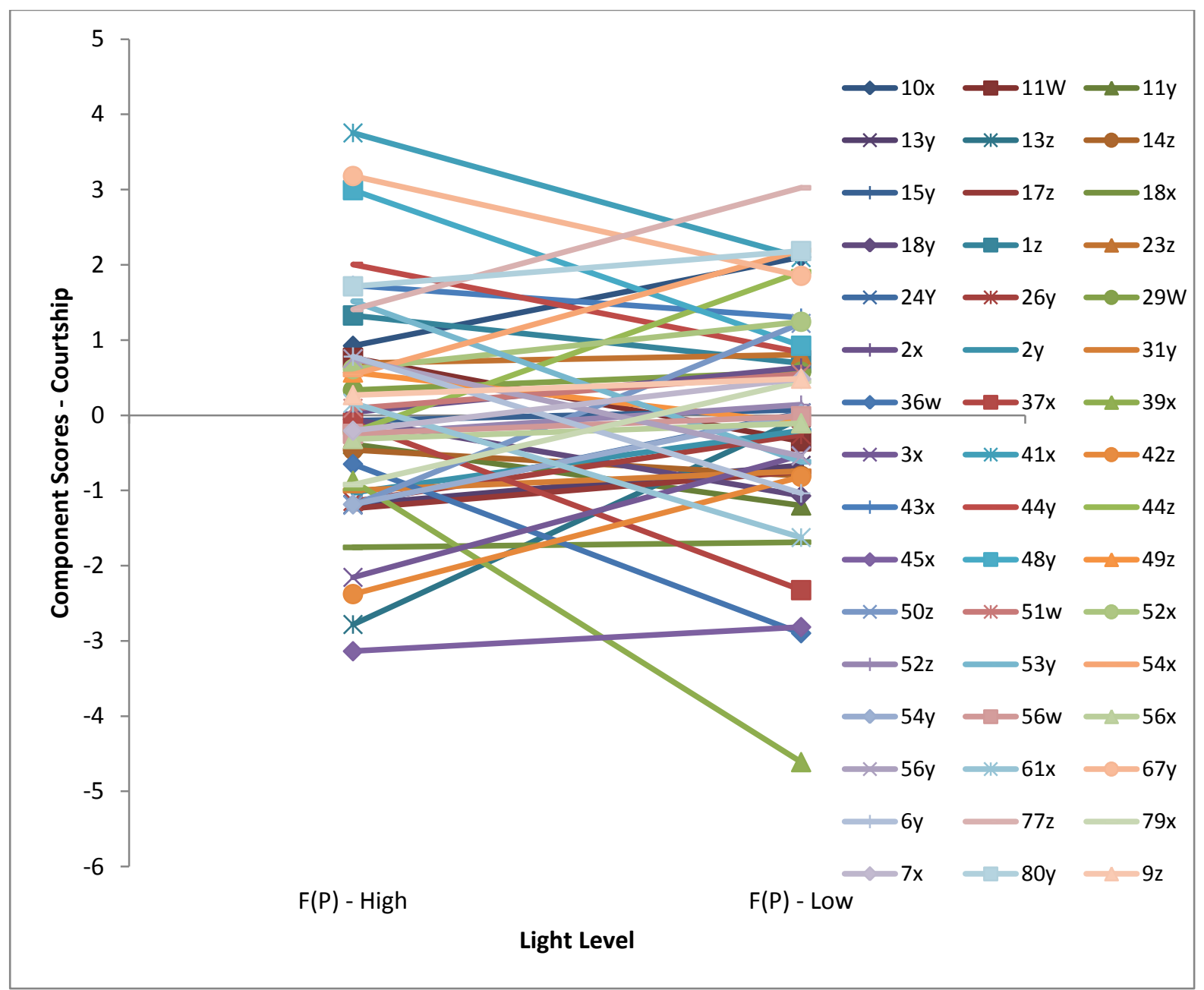

Figure 5: Reaction norm plot for female present courtship component between high and low light levels. Each male's component score in high and low light is represented by a particular line with the legend showing Male ID numbers. 


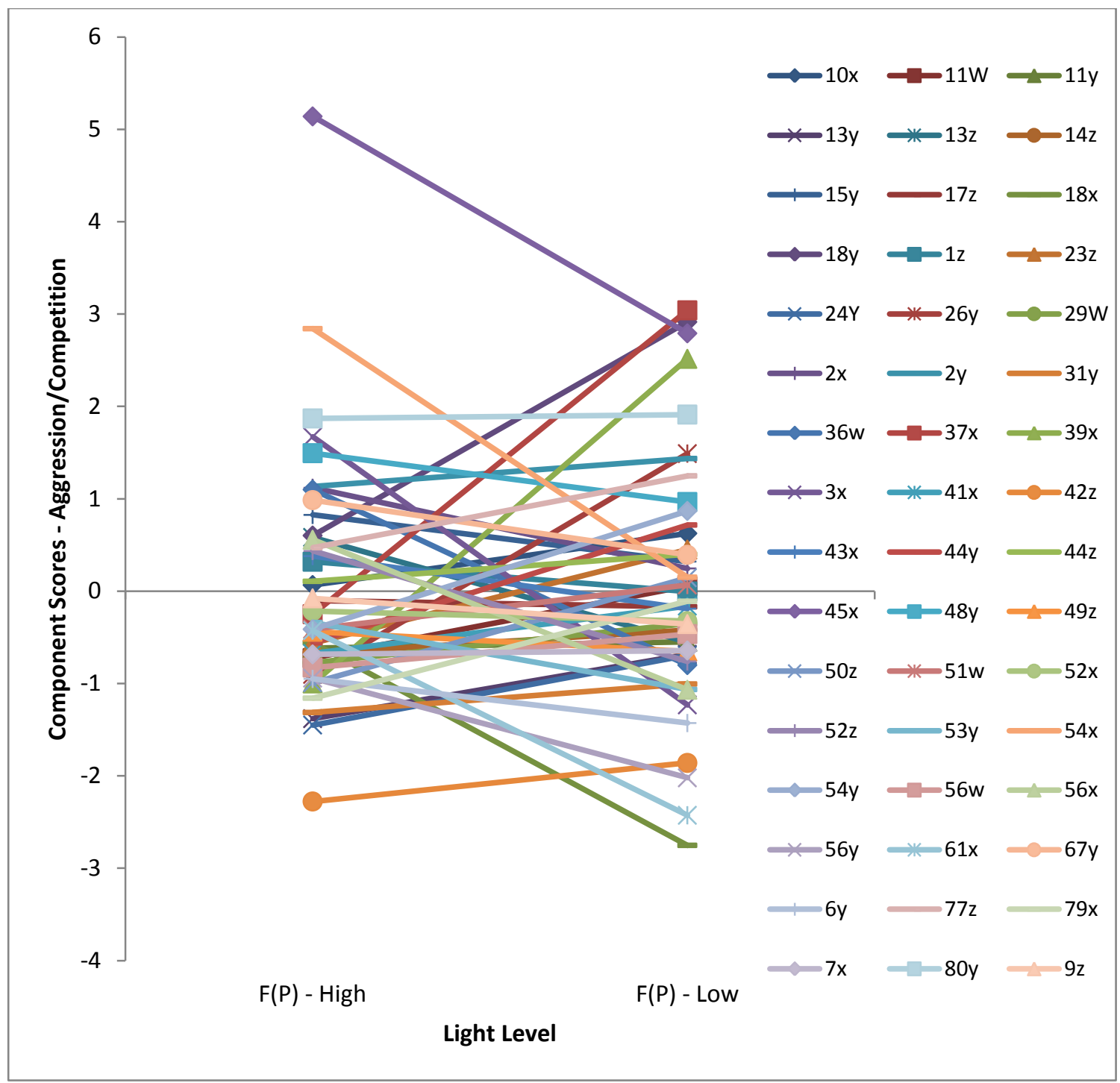

Figure 6: Reaction norm plot for female present aggression/competition component between high and low light levels. Each male's component score in high and low light is represented by a particular line with the legend showing Male ID numbers. 

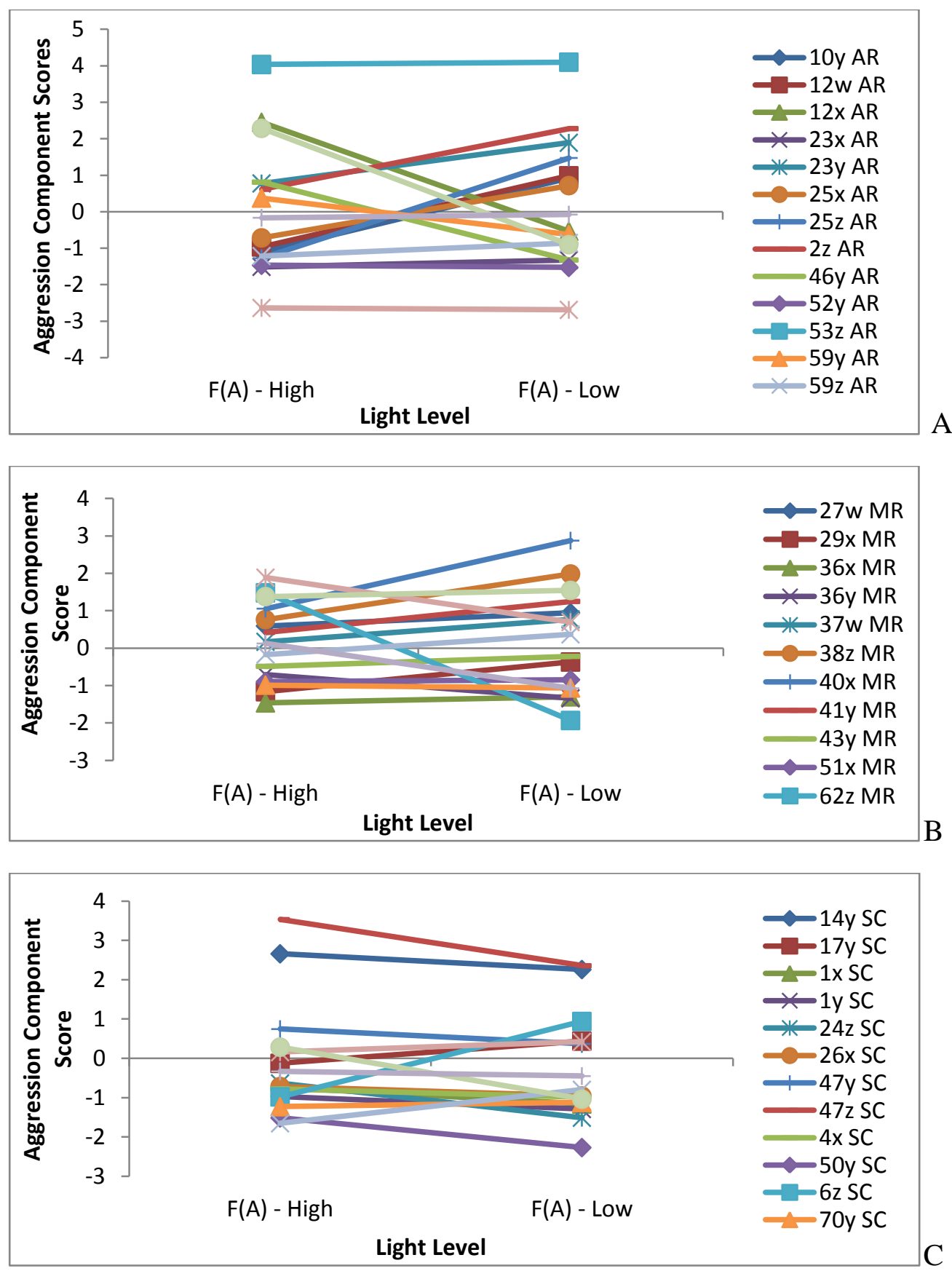

Figure 7: Reaction norm plots by population (A: Aripo; B: Marianne; C: Small Cray) for female absent male -male aggression component between high and low light levels. Each male's component score in high and low light is represented by a particular line with the legend showing Male ID numbers. 

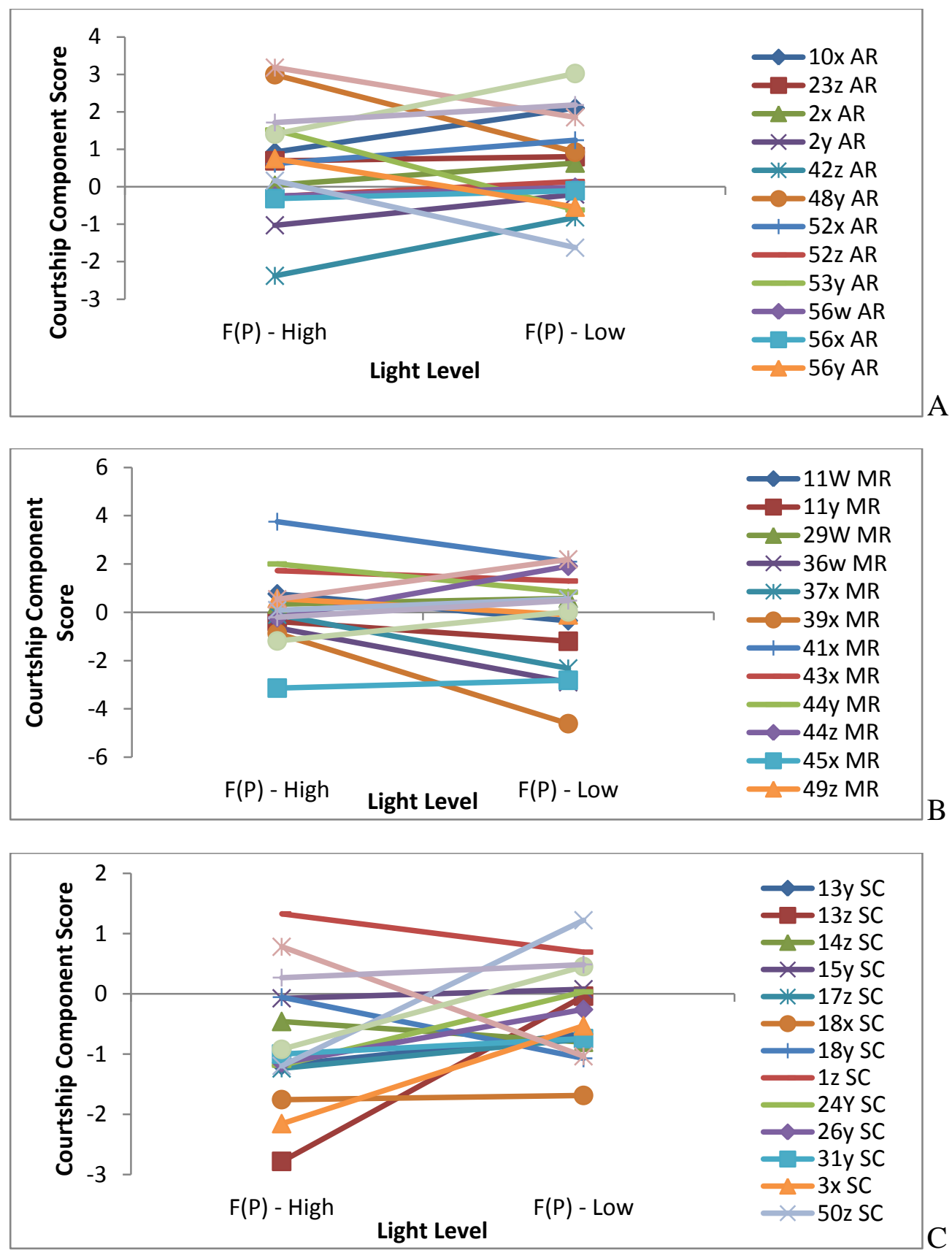

Figure 8: Reaction norm plots by population (A: Aripo; B: Marianne; C: Small Cray) for female present courtship component between high and low light levels. Each male's component score in high and low light is represented by a particular line with the legend showing Male ID numbers. 

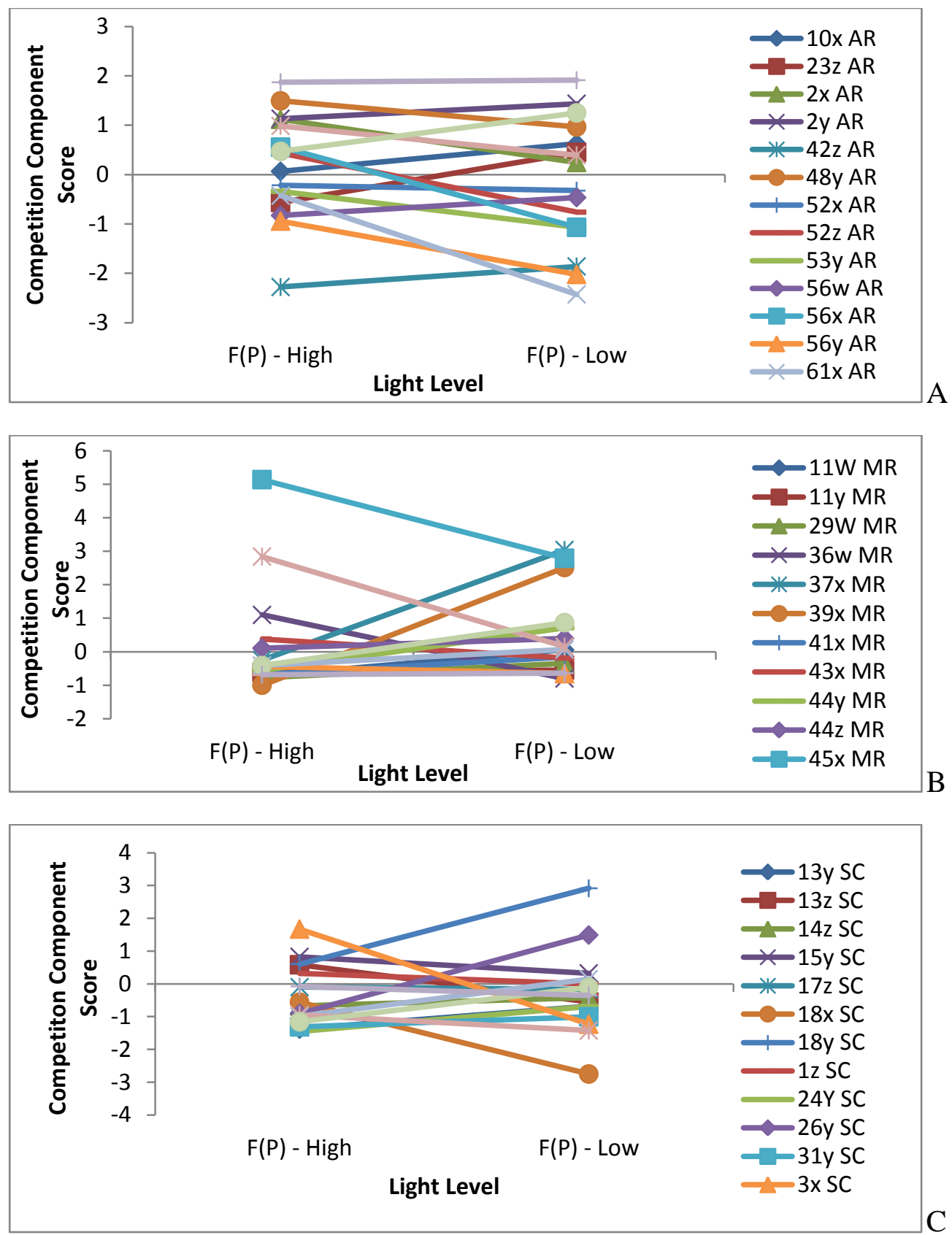

Figure 9: Reaction norm plots by population (A: Aripo; B: Marianne; C: Small Cray) for female present aggression/competition component between high and low light levels. Each male's component score in high and low light is represented by a particular line with the legend showing Male ID numbers. 
III.

\section{GUPPY AGGRESSION AND COURTSHIP AND THE RELATIONSHIP OF THESE BEHAVIORS WITH CORTISOL, TESTOSTERONE, AND 11- KETOTESTOSTERONE ACROSS LIGHT ENVIRONMENTS IN THE PRESENCE AND ABSENCE OF SEXUALLY RECEPTIVE FEMALES}

\section{Introduction}

Behavioral flexibility involves differing behavioral responses by individuals to a diversity of environmental cues, which allows an organism to produce adaptive responses to fluctuating environments, including rapid anthropomorphic changes to landscapes such as deforestation (West - Eberhardt 1989; Piersma and Drent 2003; Ghalambor et al. 2007; Schwartz and Hendry 2010; Sih et al. 2011; Snell-Rood 2013). Predation risk is one such factor that can be expected to affect behavioral patterns of prey species. Among a variety of species, light conditions can influence predation risk. In a classic example of light conditions affecting perceived predation risk, Lockard and Owning (1974) showed that banner-tailed kangaroo rats with an adequate supply of food foraged only when the lunar cycle suggested a moonless night. They concluded that predators may have more difficulty seeing their prey on moonless nights (Lockard and Owning 1974). In contrast, kangaroo rats without an adequate supply of food were not selective about the moon phase in which they foraged. In a more recent study, lunar cycles influenced the hunting patterns of an apex predator, the African lion. Packer et al. (2011) showed that African lions hunted more effectively on dark nights near a new moon, perhaps because visually orienting prey species had a harder time detecting them. Increase in nighttime brightness decreased kill numbers. Interestingly, when the nighttime light intensity was at its brightest during a full moon, lions were more likely to hunt during the day. 
Light intensity and its relationship with predation may affect a multitude of fish species as well. In many open-water systems, predator activity is increased at twilight when prey are illuminated by the light from above, and predators from the depths are hard to detect in the dark deeper waters by prey species (Munz and McFarland 1973; Parrish 1992). Schooling behavior, often associated with anti-predator behaviors in prey fish species (Magurran 1990), is affected by light intensity. Ryer and Olla (1998) postulate that if schooling is the primary defensive strategy against predation, increased swimming speed in schooling/shoaling fish species in early morning illumination may help mediate the increased risk of predation at this time because schools of fish can reform more rapidly. When complete darkness abounds and schools fall apart because visually orienting fish cannot see each other, some fish species decrease their activity to reduce the potential for detection by predators (Helfman 1993). Furthermore, artificial lighting in coastal ocean environments on man-made structures has shown to increase the abundance of predatory species, and that these artificial lighting conditions optimize conditions for predation (Becker et al. 2013). There is good evidence of an important interaction between light intensity and the intensity of predation in guppy stream systems as well (Endler 1987, Archard et al. 2009).

Guppies are small, hardy, live-bearing, tropical poeciliid freshwater fish that inhabit still pools in clear, swift flowing streams, and the edges and backwaters of small rivers in mountain forest areas of Trinidad and Tobago, Venezuela, Guyana, and Suriname (Houde 1997; Magurran 2005). Guppies occur in stream habitats varying in a variety of environmental conditions, particularly predation pressure and light intensity (Houde 1997; Magurran 2005). Guppy populations described as high predation 
populations are subjected to major crustacean and fish predators, predominately the pike cichlid Crenicichla alta, while guppy populations described as low predation populations are subjected principally to the minor gape-limited killifish, Rivulus hartii (Endler 1995; reviewed in Magurran 2005).

The behavior of guppies, especially male mating behavior, has been extensively studied and described (Haskins and Haskins 1950; Baerands, Brouwer, and Waterbolk 1955; Liley 1966). Males utilize both an elaborate sigmoid courtship display to attract and mate with willing females, and sneaky copulations in which males attempt to sneak copulations without displaying to a female first (Houde 1997; Kelly and Godin 2001; Kolluru et al. 2007). Males also engage in aggressive interactions with other males. These interactions include both competitive interactions in which multiple males competitively court the same females, and dominance interactions in which males interact aggressively with each other without females nearby, potentially to establish relationships with respect to future mating rights to receptive females (Kolluru and Grether 2005; Kolluru et al. 2007).

Male guppies flexibly adjust their behavior to variation in perceived predation risk based on light intensity, and the evidence suggest that guppies perceive high light environments as high risk environments (Endler 1987; Endler 1991; Gamble et al. 2003; Archard et al. 2009; Chapman et al. 2009). Guppy streams vary in light intensity on a temporal and spatial scale due to variation in canopy cover, time of day, weather (e.g., cloudiness), water turbidity and water depth, as well as human caused environmental disturbances such as deforestation (Endler 1987; Luyten and Liley 1995; Reznick et al. 2001; Gamble et al. 2003; Kolluru et al. 2007; Archard et al. 2009; Chapman et al. 2009; 
Schwartz and Hendry 2010). Male guppies become more conspicuous to C. alta at midday, when light intensity is greatest, than they are early and late in the day, and $C$. alta is more active and forages more intensely at high light intensities (Endler 1987, 1991). In the guppy system, the immediate effects of the light environment on behavior may occur when variation in light environment changes the conspicuousness of an individual (Long and Rosenqvist 1998; Chapman et al. 2009; Archard et al. 2009). Typically, these light changes result in an individual flexibly adjusting a variety of behaviors to take advantage of the ambient light environment or to offset the perceived risk of higher predation (Long and Rosenqvist 1998; Archard et. al. 2009). Guppies would be expected to be more conspicuous to females under higher light situations which likely would have direct fitness benefits to male guppies who courted more under high light environments. Long and Rosenqvist (1998) showed that male guppies alter their courting distance in response to ambient light conditions but also concede that courtship behavior may be constrained by selective pressures on male and females such as predation. Male guppies adjust their behavior under high predation risk conditions by performing fewer courtship displays, reducing their aggression, and attempting more sneak copulations (Endler 1987; Magurran and Seghers 1990; Magurran and Nowak 1991; Godin 1995; Houde 1997; Kolluru et al. 2007; Kelley and Brown 2011). Interestingly, males adjust their behavior in response to light levels in much the same way as to predation risk. Males perform fewer courtship displays under high (midday) light levels, perhaps in response to a perceived increased risk of predation (Reynolds et al. 1993; Archard et al. 2009). Furthermore, Archard et al. (2009) showed that reduction in conspicuous courtship under high light conditions occurs independently of time of day. 
This evidence suggests that guppies directly perceive high light as being an environment in which there is a high risk for predation.

The social environment should influence male guppy behavior as well. The way a male behaves in the absence of sexually receptive females may be quite different than how it may behave in female present social environments because of the social environments effects on the costs and benefits of behavioral tactics. When females are present, males gain direct fitness by investing in behaviors associated with reproduction including courtship and sneak copulation attempts (Kolluru and Grether 2005; Price and Rodd 2006). Inversely, when females are not present, a male may gain indirect fitness by investing in his social standing in the dominance hierarchy of the group by engaging in such behaviors as male-male aggression (Kolluru and Grether 2005; Price and Rodd 2006).

Few low predation populations have been included in studies of flexibility in male behavior. The Aripo River population, a low predation population, has been included in variety of other guppy studies but other low predation populations have rarely been included (Magurran and Seghers 1990; see Godin and Briggs 1996; Archard et al. 2009). Studying high predation sites makes sense because if selection by predators has shaped behavioral flexibility, males from high-predation populations should exhibit a stronger response to perceived predation risk than males from low-predation populations (Magurran and Seghers 1990; Templeton and Shriner 2004; Archard et al. 2009; Elvidge et al. 2014). However, low predation sites are still subject to predators, including fish predators and aerial predators like kingfishers, although at less intensity and efficiency (Magurran 2005). Understanding the extent of behavioral flexibility to light intensity in 
low predation populations would increase our overall understanding of the importance of environmental light conditions on behavior in general.

Behavioral traits are caused by both proximate and ultimate factors, and because behavior is often influenced by the proximate factor of hormones, levels of hormones should exhibit the same flexibility as the behavior that they influence (Oliveira 2009). In poeciliid fish, the androgens testosterone $(\mathrm{T})$ and 11-ketotestosterone (11KT) mediate courtship and aggressive behavior, and also, as in other species, cortisol is released as part of the stress response (Borg 1994; Dzieweczynski et al. 2006; Hallgren et al. 2006; Miles et al. 2007; Cureton et al. 2010; Gabor and Grober 2010; Fuzzen et al. 2011). Behavioral plasticity across light levels and perceived predation risk environments, as well as the response to social environment, is likely to be mediated by these hormones. In acute challenge situations (Wingfield et al. 1990; reviewed in Hirschenhauser and Oliveira 2006), androgen hormone levels should be higher than in stable conditions, enabling dominance behaviors such as aggression to be promoted when challenges to social standing occur (Oliveira et al. 2002 ; Dzieweczynski et al. 2006; Oliveira 2009). Also due to the potential stress of high risk environments, I would expect cortisol levels to correlate positively with high light levels as well as with social challenge environments.

The aim of this study was to test the following questions: How do guppies respond behaviorally to different light levels in varying social conditions when sexually receptive females are present and when they are not present, is there an underlying correlation between behavior and hormones under two different light conditions and in two different social conditions, and are there correlations between behavior and 
hormones in stable social groups and challenge situations? In so doing, I hoped to gain an understanding of how low predation populations of guppies respond to light environments under a different set of social conditions. Due to the perceived higher predation risk under high light levels, I predicted that conspicuous behavior such as male aggression and courtship should be reduced under high light compared to low light, and that female response to courtship should be reduced because females would be more susceptible to predation in this situation as well. I also predicted that male-male aggressive behaviors would be more frequent in the absence of sexually receptive females because such behavior should yield benefits such as increased dominance in the group whereas males in the presence of females, should invest in behaviors yielding more direct fitness payoffs, such as courtship and sneak copulations. There should be a positive relationship between levels of the androgens testosterone (T) and 11-ketotestosterone (11-KT) and behavior in social challenge situations based on previous studies with another poeciliid, Xiphophorus helleri (Hannes 1984; Hannes 1986). Furthermore, the T and 11-KT release rates should be greater under low light because they are expected to modulate courtship and aggression, behaviors which should be more frequent under low light as well. Cortisol levels should be higher under the more stressful high light, high predation risk scenarios as well. This study will add to the overall understanding of geographic variation in behavior and hormone flexibility, and what is understood regarding the response to light levels in low predation guppy populations.

\section{Methods}

Outline of Experimental Design: I examined the behavioral interactions of guppies as a function of social environment (Female Present versus Female Absent) in two different 
light conditions (High Light/Low Light) over the course of two days to ascertain how social and environmental conditions affect aggressive and courtship behavior among males, and the relationship between circulating hormones and male behavior.

Furthermore, I looked at the relationship between circulating hormones and male behavior in established social groups and in novel social group situations.

Study Populations: The guppies used in this experiment were laboratory descendants (within 10 generations) of wild-caught fish collected from three geographically isolated streams representing 3 different drainage systems in Northern Trinidad: the Marianne River (PS 858 895), the Small Crayfish River (PS 965 835), and the Aripo River (PS 937 803) (Grether et al. 2001). These streams were chosen during surveys of stream drainages conducted in 1996 and 2000 (Grether et al. 2001) and were based on four criteria outlined in Grether et al. (2001): 1) intact old growth rainforest, 2) relatively uniform forest canopy cover, 3) geographic isolation from each other created by multiple barriers to guppy dispersal, 4) and low predation sites with no predatory fish except Rivulus hartii, a small, gape-limited predator (Grether et al. 2001; other details about these sites are given in Kolluru et al. 2007). Although all sites are low-predation with respect to fish predators, the Marianne River contains diurnally active prawns (Macrobrachium crenulatum) (Millar et al. 2006; De Serrano et al. 2012) absent at the Aripo River (De Serrano et al. 2012) and the Small Crayfish River, although the latter site may have contained prawns prior to the construction of the Hollis Dam in 1936 (http://wasa.gov.tt/WASA_Education_water_Reservoir_Hollis.html). All three sites are also likely to be subject to a variety of bird predators (Templeton and Shriner 2004). 
Laboratory Setup: The Marianne River and Small Crayfish River fish were obtained from the laboratory of Gregory Grether at the University of California, Los Angeles in spring 2007. The Aripo River fish were obtained from Brian Smith in the laboratory of Dan Blumstein at the University of California, Los Angeles in fall 2008. All fish were transported to the Kolluru laboratory at California Polytechnic University, San Luis Obispo, California via automobile, in aerated, temperature-controlled containers.

Fish were maintained in multiple mixed-sex 10-gallon stock tanks to allow for breeding and to minimize inbreeding. These tanks contained natural, multi-colored gravel, Java moss (Taxiphyllum barbieri) to provide refuge, and trumpet snails (Melanoides tuberculata) to help maintain appropriate water conditions by consuming algae. The lab temperature was maintained at $25 \pm 0.5^{\circ} \mathrm{C}$ and the fish were exposed to 12:12 L: D cycle using full spectrum fluorescent (Philips Home Light Natural Sunlight Full-Spectrum Light; 2950 lumens; 32 watt) and LED light sources. The stock populations were fed TetraMin ${ }^{\circledR}$ Tropical flakes (Tetra Holding, Inc.) twice per day during the week and once per day on the weekends, periodically supplemented with Ocean Star® International Spirulina flakes and Hikari® frozen brine shrimp.

In April of 2008, approximately 20 healthy females from each population were individually isolated in 2-gallon plastic tanks with a healthy male from the same site, and allowed to give birth. Because the females were chosen from stock populations, they were most likely gravid by the time they were isolated; however, the companion male in each 2-gallon tank may have fathered some of the offspring. These tanks were outfitted with plastic nets that separated the tank into two sections, allowing fry to swim away from the adults, to minimize cannibalism. Each tank contained gravel, and Java moss for 
cover. These tanks experienced the same lab conditions and feeding schedule described above.

Offspring were removed from the female's tank at 1-3 weeks of age, and transferred to 2-gallon tanks containing multi-colored gravel and Java moss (Taxiphyllum barbieri), at densities of 2 to 6 fish per tank, with each tank containing representatives from no more than two litters. At approximately ten weeks of age, the juveniles were sexed and separated into single-sex 2-gallon tanks. Sex was determined by the presence of pigmentation and gonopodium development in males, and dark coloration around the anal fin in females (Houde 1997). After sexing, two types of single-sex 2-gallon tanks were set up: male tanks (Stable Social Group Tanks) contained 1 to 4 males, and separate female tanks contained 1 to 3 females. Because offspring were sexed and separated before completion of development of the gonopodium in males, I am confident that the fish were all virgins prior to observations (Houde 1997). After they were sexed and separated, males and females did not have any visible contact with each other, to minimize the influence of visual contact between the sexes on male competitive interactions and female choice (Grether 2000; Hibler and Houde 2006).

Stable Social Group Focal Observations: Observations of males in their Stable Social Group tanks were performed to establish baseline social dominance behavioral status for each male that was used in the subsequent observations that occurred between March and December 2009. I used random number generators to randomly select which males to test on a given day, with respect to population (MR, AR or SC), light treatment experienced on the first day (High Light or Low Light), social environment treatment (Females Present or Females Absent), and which of two identically outfitted observation tanks 
would be used for the subsequent behavioral observations (see below for details on these observations). Individual males to be used for the behavioral observations were chosen based on the following criteria: 1) the oldest available males; 2) males from tanks containing more than 1 male; 3 ) visibly healthy males; 4) sized matched males (determined by observing body length with the naked eye); 5) males that were born within three weeks of each other; and 6) males whose Stable Social Group Tanks were on different shelves in the laboratory, further minimizing the chances of prior visual contact. All of the male Stable Social Group tanks were given a number (e.g., “29”). Males chosen based on the above criteria for the subsequent trials were given a unique letter identifier (X, W, Y, or Z) within each tank so that each individual carried a unique ID based on the number of the tank and the letter of the fish (e.g., "29W"). The chosen male's color patterns were then sketched to enable individual identification among the fish in their Stable Social Group tanks and between the fish in the subsequent behavioral trials.

After two males from different Stable Social Group tanks were chosen, their color patterns sketched, and each fish assigned unique ID numbers, the two Stable Social Group tanks housing the chosen fish were visually isolated from neighboring tanks and the fish were fed a small pinch of flake fish food (details above). The chosen individual fish's behavior was then observed in the Stable Social Group tanks. Stable Social Group Tank focal observations (hereafter referred to as Stable Group Observations) consisted of 5-minute periods of focal animal observation, conducted at 3 times of day: 900 to 1200 hours (PST), 1400 to 1600 hours (PST), and 1800 to 2000 hours (PST). Interspersing 
observations throughout the day allowed me to capture diurnal variation in behavior patterns (Endler 1987; Reynolds et al. 1993; Kolluru et al. 2007).

Stable Group Observation trials began with an acclimation period. Within 3-5 minutes after feeding the tank housing one of the two fish, the observer stood relatively motionless, 30 centimeters from the tank for approximately 2 minutes. After the acclimation period, the observer performed focal observations on that tank. Interactions between the focal male and the other males in the Stable Social Group tank were recorded. All behaviors for the Stable Group Observations were tallied on behavior observation charts. Behavioral sequences were recorded by using a letter instead of a hatch mark when tallying. I assigned the first behavior a capital " $\mathrm{A}$ " and the subsequent behaviors successive capital letters of the English alphabet, switching to lower case letters after all upper case letters had been used. Totals were obtained by counting the total number of tallies recorded for each behavior. These data were entered into a Microsoft Excel spreadsheet. The Stable Group Observations for the second male occurred immediately after the first focal male observation period ended.

Behavioral Variables (Stable Group): For all Stable Group Observations, the following variables were recorded for each male: chase, display, bite, face-off, lateral, and scuffle (See Table 11).

Challenge Situation Tank Focal Observations: On the day after the Stable Group Observations, I performed observations to assess the behavior of males under a social challenge. For these tests, the two males observed in the Stable Group Observations, were paired with each other for the first time, for the Challenge Situation Tank Focal 
observation trials (hereafter referred to as "Challenge Group Observations"). Behavioral observations were accomplished in one of two 20-gallon observation tanks ( 30 x $12.5 \mathrm{x}$ 12.5 inches) containing natural, multi-colored gravel bottoms and plastic bubblers connected to under gravel filters. The back and sides of each tank were covered in a uniform brown paper. The temperatures in the tanks were maintained at the laboratory temperature range of $25^{\circ} \pm 5^{\circ} \mathrm{C}$ and the tanks were filtered between trials with charcoal canister filters (Marineland® H.O.T. Magnum 250 HSB Canister Filter) to minimize chemical effects on the behavior of fish in subsequent observations (Crow and Liley 1979). The observation tank area was blocked off from the rest of the laboratory (including the general room lighting) by a heavy black curtain that extended completely around the tanks. Two sets of full-spectrum florescent lights (Vita-Lite $® ; 30$ watt; Duratest 07-15121) and several sets of LED lights spanned the length of the two observation tanks. The lights were mounted 22.86 centimeters above each tank, and each set of lights included two full spectrum light tubes that stretched 91.44 centimeters across, providing even illumination over the two tanks. Small strips of LED lights were placed between each set of fluorescent lights. Fish were fed TetraMin ${ }^{\circledR}$ Tropical Flake food to satiation 15 minutes before the first observation period and immediately after the second observation period, to minimize the effects of competition for food (Magurran and Seghers 1991).

Each male was observed under both Low Light and High Light levels (on subsequent days, in a randomized order) and in one of the two social environment treatments (Females Present or Females Absent). Light levels were measured using a Li250A Quantum/Radiometer/ Photometer light meter (Li-Cor Biosciences Inc.) equipped 
with a Li-190SA quantum sensor, and all readings were taken in the middle of each tank and approximately 2.54 centimeters above the water surface. The Low Light level was defined as 5 to $25 \mu \mathrm{mol} / \mathrm{m}^{2} / \mathrm{s}$ and the High light level as 70 to $90 \mu \mathrm{mol} / \mathrm{m}^{2} / \mathrm{s}$. These values were based on field measurements of photosynthetically active light levels above Trinidadian guppy streams (Grether et al. 2001; Kolluru and Grether 2004; Kolluru et al. 2007), and on previous laboratory studies of behavioral plasticity across light levels (Reynolds et al. 1993), as well as the lowest light levels under which behaviors could be seen. High light was achieved by turning on all observation tank lights, and Low light conditions were achieved by turning on all observation tank lights and placing a double layer of black shade cloth over the top of the observation tanks which reduced irradiance at the water level.

Challenge Group Observations occurred between April and December 2009. The two focal males chosen based on the criteria described above in the Stable Group Observations were observed with each other for the first time the next day. A trial was initiated by netting the males from their home tanks, collecting Hormone Sample 1 (see Hormone Level Measurements description below), setting the proper light conditions for Day 1 over both tanks, allowing the two males to acclimate to observation tank water conditions for 5 minutes in two separate clear caddies, and releasing the two males into one of the two observation tanks, chosen at random, between 900 and 1100 hours (PST). If the social treatment was "Females Present", two size-matched, healthy females from two different female Stable Social Group tanks were chosen from virgin female tanks of the same site as the males. The females were acclimated to water conditions for 5 minutes in two separate clear caddies and released into the observation tank with the males. After 
releasing the fish, we fed them a small pinch of flake fish food, and closed the curtain, visually isolating the observation tanks from the rest of the laboratory.

Observation sessions began between 1500 and 2000 hours (PST), the same day the fish were introduced into the observation tanks. The fish were fed approximately 15 minutes prior to observations. I performed 3 five-minute observation periods per male with a minimum of 15 minutes between consecutive focal observations on a given male. Males were observed alternately, in a randomly chosen order. The fish were fed a small amount after the second focal observation. After the observations were complete on Day 1, Hormone Sample 2 was collected, the lights were turned off and the curtain closed around the observation tank area.

The second day of observation ("Day 2") began between 900 and 1100 hours (PST). The light conditions were changed to the opposite of what they were on Day 1, the fish in the observation tank were netted and placed in their respective beakers, the curtain was closed around the observation tank area, and Hormone Sample 3 was collected. After the Hormone collection period was complete, the fish were replaced in the appropriate observation tank and the fish were fed. Observations for Day 2 occurred between 1500 and 2000 hours (PST) and followed the same pattern described above for Day 1. After the conclusion of observations on Day 2, Hormone Sample 4 was collected, and the fish were anesthetized using MS 222 (tricaine methanesulfonate; 200mg/L of water; Finquel; Argent Chemical Laboratories), weighed to the nearest $0.1 \mathrm{mg}$, their standard length was measured to the nearest $0.01 \mathrm{~mm}$ using digital calipers, and digital images of the left and right lateral surfaces were taken for future analyses of color patterns. Males were allowed to recover in a 2 gallon clear tank filled with fresh regular lab water set aside for this 
purpose. After sufficiently recovering they were placed into their specific population stock tanks.

All Challenge Group Observation data were recorded on a Macintosh Power Book G4 computer (OSX operating system; Apple) using a True Basic Silver Edition behavior event-recording program written by J.C. Walz, based on a program written by G.F. Grether.

We conducted a total of 48 trials, resulting in observations on 96 males $(\mathrm{n}=$ Marianne River, 32; Small Crayfish River, 32; Aripo River, 32). Half of the trials ( $\mathrm{n}=24$ trials) involved the social treatment "Females Absent" ( $\mathrm{n}=$ Marianne River, 16; Small Crayfish River, 16; Aripo River, 16) and the other half, with equal sample sizes of males and trials, involved the "Females Present" social treatment.

Behavioral Variables: Behaviors recorded were taken from Kolluru and Grether (2005), with the addition of the lateral display and face-offs (see Table 12). For all Challenge Situation Tank Focal observations the following variables were recorded for each male: follow, nip, sigmoids, competition, sneaks, swings, switch, display, competition/dominance, chase, scuffle, bite, lateral display, foraging, face-off and moving. In focal observations in which females were absent the following behaviors were excluded since they are exclusive to male behavior towards females: sigmoids, competition, competition/dominance, follow, and nip.

Hormone Sampling: Hormone samples were collected from each male using a noninvasive water-borne technique (Scott et al. 2008; Wong et al. 2008). For each of the four hormone samples referred to above, each male was netted and placed in a 250-ml beaker 
with $200 \mathrm{ml}$ of conditioned water at the lab temperature of $25^{\circ} \mathrm{C} \pm 5^{\circ} \mathrm{C}$ for 60 minutes. A separate $250 \mathrm{ml}$ beaker with $200 \mathrm{ml}$ of clean conditioned water, but with no fish, was used as a control and placed next to the beakers containing the fish. The order in which the fish were netted or poured out of the beaker into a net was chosen at random for all hormone collections procedures. All beakers were cleaned prior to use with soap and water, and rinsed thoroughly with reagent alcohol.

The beakers with the fish and the control water beaker were covered by a single sheet of white printer paper to keep the fish from jumping out of the beakers. Several pencil-sized holes were made in the paper to allow for air exchange. The beakers were placed inside the Challenge Group Observation tank area, light levels were adjusted to the levels the fish were to experience on Day 1 of the Challenge Group Observation (see behavior observation description above), and the curtain was closed around the observation tank area. The fish were removed from the beaker after 60 minutes by pouring the water through a net into an empty, clean beaker; I thereby captured the fish in the net and added it to the appropriate acclimation or observation tank. The water was immediately poured into one $250 \mathrm{ml}$ or four $50 \mathrm{ml}$ plastic vials, and stored at $-80{ }^{\circ} \mathrm{C}$ until hormone extraction.

Initially, water samples were shipped in temperature controlled containers under dry ice to the laboratory of Dr. Ryan Earley at the University of Alabama for complete hormone extraction and assay. However, after approximately the first complete set of samples for 10 males, I elected to perform hormone extraction, and send columns containing extracted hormones (described below) to the Earley lab for quantification assays. I performed solid phase hormone extraction with C18 columns. All final elutions 
and assays were performed in the laboratory of Dr. Ryan Earley at the University of Alabama. Water samples were thawed for 12 hours at room temperature prior to the $\mathrm{C} 18$ column extractions. The water was filtered through Whatman Filter paper (Grade 1, 24 $\mathrm{cm})$ to remove any particulate matter, into appropriately labeled $250 \mathrm{ml}$ beakers, in preparation for hormone extraction, which was achieved using C18 solid phase extraction (SPE) columns (Waters, Inc.; Certified SepPak® Vac C18 3cc/500 mg) fitted to a 12-port vacuum manifold. The columns were primed with two consecutive washes of $2 \mathrm{ml}$ of HPLC-grade $100 \%$ Methanol $(\mathrm{MeOH})$ followed by two consecutive washes with $2 \mathrm{ml}$ distilled water. Tygon® tubing (Saint Gobain formulation 2275) wrapped with Parafilm (Bemis Company, Inc.) was fitted to each column and placed into the corresponding water sample beaker. The $200 \mathrm{ml}$ water samples were then passed to the columns via the Tygon ${ }^{\circledR}$ tubing and pushed through the columns slowly (drip by drip) using the vacuum manifold. After completely drawing the samples through the columns, the C18 columns were immediately removed from the manifold, covered by Parafilm, and frozen at $-80^{\circ} \mathrm{C}$. All columns produced at the Kolluru lab were shipped in temperature-controlled containers under dry ice to the Earley lab for elution and radioimmuno ELISA assay for testosterone, cortisol, and 11-ketotestosterone hormone levels in each sample.

What follows is a description written by a collaborator Ryan Earley: Water-borne hormone samples shipped to the University of Alabama were stored at $-80^{\circ} \mathrm{C}$ until extraction; columns were stored at $-20^{\circ} \mathrm{C}$ until elution (see elution protocol below). After extractions performed in the Earley lab, the C18 columns were washed with 2 consecutive washes of $2 \mathrm{ml}$ distilled water to purge salts (Earley et al. 2006). Samples that arrived as C18 columns after the extraction was performed in the Kolluru lab were 
thawed overnight at $4^{\circ} \mathrm{C}$ and then washed with 2 consecutive washes of $2 \mathrm{ml}$ distilled water. Free hormones were eluted from all the C18 columns into borosilicate vials using 2 consecutive washes of $2 \mathrm{ml}$ ethyl acetate (Ellis et al. 2004). Conjugated hormones (sulphated and glucuronidated) were eluted from all the columns into separate borosilicate vials using 2 consecutive washes of $2 \mathrm{ml}$ HPLC-grade methanol (Ryan Earley per.comm.).These eluted samples were stored at $-20{ }^{\circ} \mathrm{C}$ until being processed further. The samples were dried under a flow of nitrogen using an Evap-O-Rac (ColeParmer) in a water bath at $37^{\circ} \mathrm{C}$, which resulted in a hormone residue. Residues were resuspended in $30 \mu \mathrm{l}$ ethanol and vortexed for $1 \mathrm{~min}$, and then $570 \mu \mathrm{l}$ enzymeimmunoassay (EIA) buffer (provided with the EIA hormone assay kits) was added to each sample, and vortexed for $45 \mathrm{~min}$. Resuspended samples were stored at $4^{\circ} \mathrm{C}$ overnight, and assayed on the following two days.

To assay cortisol, testosterone (T), and 11-ketotestosterone (KT) from each sample, EIA Assay kits (Cayman Chemicals, Inc.) were used and kit instructions were strictly adhered to (details are provided in Lorenzi et al. 2008). All hormone samples were analyzed blind to treatment, and in duplicate together on sixteen 96-well plates per hormone (48 plates total). Furthermore, each plate had a set of controls run in duplicate at the beginning and end.

The pooled control was generated by combining $30 \mu \mathrm{l}$ of hormone resuspension from $524 P$. reticulata samples (experimental animals). Intra-assay coefficients of variation on the 16 plates ranged from $1.5-4.6 \%$ (median: $2.8 \%$ ) for cortisol, $1.7-16.5 \%$ (median: $3.3 \%$ ) for T, and 1.8-7.0\% (median: $4.3 \%$ ) for KT. Inter-assay coefficients of variation were $7.1 \%$ for cortisol, $5.8 \%$ for $\mathrm{T}$, and $9.9 \%$ for $\mathrm{KT}$. 
Times for the reading of plate development were based on manufacturer instructions and previous analyses (R.L. Earley, unpubl. data). Ultimately, the development time chosen for statistical analysis was based on a combination maximum binding $\left(\mathrm{B}_{0}\right)$ subtracted values (within range 0.6-1.3) and the highest $\mathrm{R}^{2}$ values for standard curves. Assays were validated for guppies by generating serial dilutions of the P. reticulata pool (1:1 through 1:64) and assessing parallelism to the standard curve. Slope comparisons were evaluated using Zar (1996, p. 355) and slopes for all hormone serial dilution curves were statistically equivalent to the standard curves (Cortisol: $\mathrm{t}_{12}=$ $\left.0.007, \mathrm{p}=0.99 ; \mathrm{T}: \mathrm{t}_{12}=0, \mathrm{p}=1.0 ; \mathrm{KT}: \mathrm{t}_{12}=0.68, \mathrm{p}=0.51\right)$.

Data Analysis: A Principle Components Analysis (PCA) was performed to reduce our behavioral variables into meaningful axes of behavior (see chapter two for complete results of the PCA). Behavioral indices were then created based on the components of the PCA to reflect important behaviors in the guppy system. To create the indices, behaviors with PCA factor loadings of +/- 0.4 were retained within the resulting PCA axes. These behaviors were than multipled by +1 or -1 based on whether the loadings for that behavior were positive or negative. These behaviors were then added together to create indices that reflected the PCA behavioral axes. Chase was analyzed separately and excluded from any of the above indices due to its conflicting units of measurements. To more closely approximate normality of residuals, the female response to courtship variable was arcsine square-root transformed, and the remaining behavioral indices were square root transformed. The hormone variables were all log transformed, and extreme outliers were removed prior to analysis. All data analyses were performed using JMP Pro 10.0.1 software (SAS® Institute, Inc. 2012). 
The first light level (High or Low light) that each male experienced was randomized, such that half of the males experienced high light level on the first day and half experienced the low light level on the first day. Any order effects of the randomized light levels (Dochtermann 2010) would emerge in our models as a significant Day $\times$ Light level interaction.

A series of repeated measures ANCOVAs was performed. To evaluate the effects of light level and social environment on male-male interactions in the Challenge Group Observations, we constructed ANCOVAs with Light level (High or Low) and Social environment (Females Present or Females Absent) as fixed effects, and mass as the covariate. We performed separate ANCOVAs for chases/bites between males and maledirected display rate and corrected for multiple test by Bonferroni correction ( $\alpha$ corrected $=0.03$ ). To evaluate the effects of light level on mating behaviors and female response in the Challenge Group Observations (Females Present treatment only), we constructed ANCOVAs with Light level (High or Low) and the repeated measure Male ID as fixed effects, and mass as the covariate. We performed separate ANCOVAs for courtship display rate, sneak copulation rate, competition instigation rate and female response to courtship and corrected for multiple test using a Bonferroni correction $(\alpha$ corrected $=$ $0.01)$.

To determine the relationships between hormone levels and behavior in Stable Group Observations, we performed separate analyses of covariance (ANCOVAs) for chases/bites between males and male-directed displays, with Population as the fixed effect and mass and the levels of each of the three hormones as measured in Hormone Collection 1 as covariates. 
To determine the relationships between hormone levels and behavior in Challenge Group Observations, we performed a series of ANCOVAs. For courtship displays, sneaks and competition instigations, the models included the fixed effects Population and Light Level and the covariates Mass and levels of the three hormones from either Hormone Collection 1 (for behavior on Day 2) or Hormone Collection 3 (for behavior on Day 3). For chases/bites between males and male-directed displays, the models included the fixed effects Population, Light Level and Social Environment, and the covariates mass and the three hormone levels from either Hormone Collection 1 (for behavior on Day 2) or Hormone Collection 3 (for behavior on Day 3). In all cases, Male ID nested within Pair ID was included as a random effect. We performed separate analyses for Day 2 and Day 3 of behavior observations, so that we could use each day's initial hormone levels (Hormone Collections 1 and 3, respectively) as covariates.

We evaluated the effects of light level and social environment on circulating levels of T, 11-KT and cortisol at Hormone Collections 2 and 4 by constructing ANCOVAs with Light level, the repeated measure Day, and Social Environment (Females Present or Females Absent) as fixed effects, and initial hormone level (the level at either Hormone Collection 1 or Hormone Collection 3) and mass as covariates.

\section{Results}

\section{Effects of the light level and Female Absent social environment on behavior}

In the Females Absent treatment, there was no effect between High Light and Low Light environments in the aggressive behavioral index $\left(F_{1,41}=1.27 ; P=0.27\right.$; Table 13, Figure 10) and there was no effect between High Light and Low Light environments 
among populations in their aggression levels $\left(F_{2,40}=1.87 ; P=0.17\right)$. There was also no effect between light environments in males chasing other males in Female Absent social environments $\left(F_{1,41}=0.04 ; P=0.84\right.$; Table 13 , Figure 11$)$. There was a significant day by light level interaction for chasing in Female Absent social environments $\left(F_{1,40}=4.70\right.$; $P=0.03)$. Males in high light environments on day 1 of the experiment were more likely to chase other males than if they were in low light environments on day 1 . On day 2 , however, males were more likely to chase other males if they were in low light environments. All other interactions were not significant $(P>0.10)$.

\section{Effects of the light level and Female Present social environment on behavior}

In the Females Present social environment, males exhibited a marginally significant difference between High Light and Low Light environments in competition instigation $\left(F_{1,43}=4.21, P=0.05\right.$; Table 14, Table 15, Figure 13) and courtship behavioral index $\left(F_{1,42}=4.86, P=0.03\right.$; Table 14 , Table 15, Figure 12) before Bonferroni correction. Competition instigation by males was higher in the Low Light treatment and was significantly different among populations. Aripo River fish instigated competitions significantly more than Small Crayfish river fish but not significantly more than Marianne River fish (Figure 17). Courtship behavior was higher in Low Light environments than in High Light environments in all populations (Figure 16). There was a slightly non-significant difference in the rate of sigmoid displays performed by males in High Light versus in Low Light $\left(F_{1,42}=3.91, P=0.055\right.$; Figure 15$)$ before Bonferroni correction but the trend was in the right direction. Males performed more sigmoid displays in Low Light then High Light environments (Figure 15). Female response to male sigmoids was significantly different between light levels and population $\left(F_{1,41}=\right.$ 
$6.17, p=0.017$; Table 14, Figure 14, 18). Females responded to male sigmoids more frequently under Low Light conditions and Marianne River females and Aripo River females responded much more frequently to courtship displays than Small Crayfish River females in general (Figure 18).

\section{Relationship between hormone levels and behavior in Stable Social Group observations}

I found no influence of hormone release rates on male-male aggressive behaviors, and no difference among populations in these behaviors, under the Stable Social Group conditions (all $P>0.10$ ).

\section{Relationship between hormones and behavior in Social Challenge Observations}

There was no relationship between testosterone, cortisol, or keto-testosterone on behavior in either Female Absent or Female Present social situations (all $P>0.10$ ).

\section{Effects of experimental conditions on circulating hormone levels of males}

For all three hormones (cortisol, testosterone, and 11-KT), there was a positive relationship between baseline hormone levels and levels after behavior observations (i.e., between Hormone Collection 1 compared to 2 on day 1 and Hormone Collection 3 compared to 4 on day 2 , respectively (T: $F_{1,141}=61.38, P<0.0001 ; \mathrm{KT}: F_{1,154}=30.56$, $P<0.0001$; cortisol: $\left.F_{1,156}=33.43, P<0.0001\right)$.

Among the three populations, only testosterone release rates were significantly different among populations $\left(F_{2,71}=3.36, P=0.04\right)$, such that Marianne River males exhibited the lowest values, Small Crayfish River males the highest values, and Aripo 
River males intermediate values not different from the other populations (Tukey's HSD; Figure 19). All other hormones levels did not differ among populations $(\mathrm{P}>0.09)$

Cortisol and 11-ketotestosterone hormone levels differed significantly between testing Day 1 and 2. Males produced higher KT levels $\left(F_{1,77}=14.68, P=0.0003\right)$ and higher Cortisol levels $\left(F_{1,116}=5.9, P=0.017\right)$ on Day 2 than on Day 1. Testosterone did not differ between testing days $(\mathrm{P}>0.05)$.

Between social situations, only cortisol levels differed, such that male cortisol levels were higher in Female Absent social situations than the Female Present social situations $\left(F_{1,70.1}=6.61, P=0.012\right.$; Figure 20$)$. Cortisol levels also exhibited a significant population by day interaction $\left(F_{2,70.9}=10.00, P=0.0001\right)$, because males from the Small Crayfish and Marianne Rivers exhibited higher values on Day 2 than on Day 1, whereas males from the Aripo River exhibited slightly higher values on Day 1 than on Day 2. Finally, cortisol levels exhibited a Social Environment x Population x Day interaction $\left(F_{2,68}=4.48, P=0.015\right)$ because on day 1 for two of the three populations, cortisol values were higher in the Females Absent treatment than the Females Present treatment, whereas for Small Crayfish males the values were almost identical in the two social environments. On Day 2 as compared to Day 1, cortisol values declined for both social environments for Aripo River males, whereas they increased in both social environments for Small Crayfish males, and were mixed for Marianne River males. Testosterone and 11-KT was not significantly different between social situations $(\mathrm{P}>0.1)$. 


\section{Discussion}

Guppies in this study exhibited behavioral plasticity in response to light level. These results occurred only in social environments in which females were present. Males reduced visually conspicuous courtship and competitive behavior, and females were less responsive to courtship, under high light levels than under low light conditions. There was a clear trend as predicted, that males courted females less under high light levels. This is consistent with previous studies (Reynolds et al. 1993; Reynolds 1993; Archard et al. 2009), and suggests that males respond to changes in light environment and/or the female behavioral response to males under different light conditions(but see Kelley et al. 2013 for a recent contrary finding).

Fluctuations in light environment have previously been shown to affect courtship behavior in guppies (Long and Rosenqvist 1998;Gamble et al. 2003). Light environments can vary between and within guppy habitats as well as with time of day and cloud cover (Endler 1987; Archard et al. 2009). For example, relatively low light conditions occur regularly at dawn and dusk, in cloudy conditions, and also in physical habitats dominated by overhanging vegetation (Endler 1987; Long and Rosenqvist 1998; Grether et al. 2001; Reznick et al. 2001). High light conditions occur at midday, but also in streams with open forest canopies (Endler 1987; Long and Rosenqvist 1998; Grether et al. 2001; Resnick et al. 2001). Reduction in conspicuous displays by male guppies under high light conditions may be an adaptive response to predation risk. For example, Endler (1987) showed that high light levels occur at times of day when guppy predators are actively foraging the most intensely. High light levels under natural and lab conditions have also been shown to elicit a decrease in conspicuous behavior similar to reductions in conspicuous displays 
elicited from the presence of actual predators (Luyten and Liley 1985; Endler 1987; Magurran and Seghers 1990; Reynolds et al. 1993; Archard et al. 2009). This study was consistent with Reynolds (1993), in that males tended to court less, and female response to courtship displays was lower, under high light conditions as well. The results further support the idea that high light does indeed represent a high-risk situation to guppies. Reynolds et al. (1993) used similar light levels as was used in this study, and found reduced courtship under high light. Our study found a clear trend in this same direction.

Interestingly, males in this study only reduced conspicuous behavior in high light conditions when females were present. To my knowledge this is the first study with guppies to look at differences in male conspicuous behavior under two light conditions in the presence of sexually receptive females and in their absence. The benefits of different male behavior, including energetically costly and conspicuous behaviors such as chasing or courtship displays, are likely to fluctuate as a function of the current environment, including the current social environment. Jirotkul (1999b) showed that male biased social situations influenced both courtship and male-male competition. Males in social situations with high male biased sex ratios courted less and for shorter periods of time but increased their competition rates. The operational sex ratio in the wild is often strongly male biased due to low female receptivity (Houde 1988; Jirotkul 1999b), and males would be required to compete more for the limited supply of receptive females. In situations in which females are present, it follows that increased competition would potentially increase direct fitness by influencing male mating success, because a dominant male has more opportunities to successfully court females (Jirotkul 1999a; Jirotkul 1999b). 
Jirotkul's (1999b) study included females in all trials, a marked difference from this study in which half our males experienced social environments completely devoid of females. In the wild, males may find themselves isolated in pools devoid of females, or in social situations without receptive females. It is possible that those males in social environments without receptive females compete with each other to establish dominance hierarchies. A male who establishes dominance in a social environment devoid of females may be better able to court females first if females became available, and may be better able to compete with other males for those females (Filby et al. 2010). The establishment of a dominance hierarchy, which could increase a male's potential direct fitness when females become available, may outweigh the risk of predation in male only social conditions. Furthermore, it is possible that the conspicuousness of male aggression in high predation situations without females is not as costly to a male's fitness as it would be were females present. When females are present it is far more beneficial for a male in high predation situations to be reserved in his conspicuous behaviors in order to avoid attracting a predator which could dissuade a female from responding to male displays. This study clearly supports the idea that the response of females to males is likely to drive a male's behavior because the light environment only influenced male behavior when receptive females were present.

Variation in female behavior under different light conditions may occur due to predation risk because females are at risk if they respond to courting males (Reynolds 1993; Pocklington and Dill 1995; Dill et al. 1999; Kelly and Godin 2001). Females have been found to respond to predation risk by reducing their response to courtship, and reversing their preference for colorful and larger males under high-risk conditions 
(Reynolds 1993; Gong and Gibson 1996; Godin and Briggs 1996; Gong 1997; Kelly and Godin 2001). It is possible that female behavior under different light conditions may help drive male behavior under different light conditions (Godin and Briggs 1996; Gong and Gibson 1996; Dill et al. 1999). In this study, competition instigation for females by males was reduced under high light conditions, which may have been a result of reduced female responsiveness to courtship displays under high light. Furthermore, males only reduced the frequency of potentially risky aggressive behavior under high light levels when in the presence of sexually receptive females, a result suggesting that males could be responding not only to the risk of predation under high light situations, but also to the decrease in female response under high light situations.

In this study I looked at behavioral plasticity in three low predation populations. Among these sites, Aripo River males instigated the most competitive events. These fish instigated significantly more competitive events than the least competitive Small Crayfish River males. There could be historical differences in sex ratio in these two populations such that the Aripo River population experienced heavily male biased situations and the Small Crayfish River populations more female biased situations. As a result these sex ratio differences may have evolutionarily influenced behavioral strategies expressed by these two populations (Jirotkul 1999b; Pettersson et al. 2004). If that is true, males evolved more competitive behavioral expression in the Aripo populations due to the relative lack of sexually receptive females whereas Small Crayfish populations do not need to engage in potentially costly competitive interactions with other males to gain access to females and thus fish from this population express relatively less competitive behaviors (Jirotkul 1999a; Jirotkul 1999b; Pettersson et al. 2004; Magurran 2005). I 
would not expect that the result of this study is a product of a lab bias as all males in this experiment experienced the same operational sex ratio, and all females used were virgin and most likely sexually receptive. Alternatively, male traits, such as the percentage of a male's body covered by orange coloration, may influence male-male competition (Jirotkul 2000). Jirotkul (2000) found that at $15 \%$ orange body coverage a male engaged in more male-male competitive and interference events than those with less orange body coverage. Although our males were visually size matched, I did not measure orange coloration of our males or match tested males for percentage of orange body coverage. Thus it is possible that the Aripo population males had larger body areas covered by orange and thus engaged in more competitive events than the Small Crayfish population males used in this study.

Females of Aripo and Marianne River populations responded to courtship more readily than Small Crayfish females. Courtship rate did not differ among populations, however. This is the first study to look at behavioral flexibility in response to predation risk indicators in fish from the Marianne River and the Small Crayfish River. Magurran and Seghers (1990) previously examined behavioral differences between high predation populations from the Aripo River proper and low predation populations from the Naranjo tributary of the Aripo River. That study suggested that changes in courtship behavior were related to the degree of predation experienced by the respective wild populations from which they were derived such that low predation populations from the Aripo River did not adjust their courtship behavior under direct predator threat. Studies of other low predation populations, such as populations from the Paria River, have found conflicting results in how guppies, particularly females, respond under direct predation threat. Godin 
and Briggs (1996) did not find differences in female response to male courtship behavior, while Gong and Gibson (1996) did find significant differences. Gong and Gibson (1996) suggest that the general response of both high and low predation populations adjusting conspicuous behavior in the presence of predators may be an ancestral response to large fish predators or just a generalized response to perceived danger. It is important to note that this study suggests that further investigation into the behavioral responses of low predation populations to conditions suggesting high predation risks are warranted.

This study was designed to be an integrative exploration of behavior and a potential proximate mechanism driving behavior, hormone release rates. This approach is important to understanding how and why an animal behaves as it does because behavioral traits are influenced by both proximate and ultimate factors (Alcock 2005). I expected to find a positive correlation between behavior and hormone release rates. I found no relationship, however. Several studies have shown that androgen release rates may be affected by interactions among conspecifics, including very short-term interactions (Borges et al. 1998; see Oliviera et al. 2002; Dzieweczynski et al. 2006; Earley and Hsu 2008). Furthermore, correlations between androgens and behavior appear to be stronger under socially unstable periods such as during the formation of dominance hierarchies, and may become dissociated during periods of social stability (Oliveira et al.2002). With this in mind, it is interesting to note that I did not find any such correlations between androgens and behavior because the social challenge situation in our experiment created a period of social instability in which males were expected to be building dominance hierarchies, and when sexually receptive females were present, males were competing for females. In cichlid fish, Borges et al. (1998) found correlations of 11-KT with sexual 
behavior after only short-term (within the course of a few hours) interactions between males and females. Oliviera et al. (1996) showed that androgen levels are socially modulated in males during male -male interactions such as establishing territories. The study collection methods should have captured any modulation in androgen levels if indeed 11-KT and testosterone correlate with behaviors in our study populations, but I did not find any modulations. Perhaps the modulation of the androgens in guppies is more subtle, and our test was not sensitive enough.

Several studies have found that androgen levels don't necessarily rise after social interactions at all, however. Dzieweczynski (2006) found that 11-KT levels which were expected to rise after interaction between Siamese fighting fish was not necessarily supported and levels rose or fell based on the audience watching an interaction and not necessarily the interaction itself. Dziewecynski (2006) concluded that 11-KT levels fluctuated with how much a male had potentially invested in an interaction. Also Dziewecynski (2006) suggests that high androgen levels may not be advantageous to males in a courtship phase as it may cause maladaptive aggressive interactions with females. In my study the threat of predation simulated by light conditions may also have affected the androgen release rates as aggressive interactions in high predation situations may similarly be maladaptive. Perhaps in this study social environment affected the expected positive correlation with aggressive and courtship behavior.

Several studies have also shown no interaction between behavior and circulating androgen levels in general. Damassa et al. (1977) found no relationship between circulating testosterone levels and sexual behavior in rats, and concluded that the levels of testosterone required to drive sexual behavior were lower than the normally circulating 
levels of the androgens in rats. Damassa et al. (1977) showed that the "threshold" of testosterone required to initiate sexual behavior was already present and therefore there was no correlated relationship with testosterone and behavior. This same relationship may exist in guppies. In a more recent study involving rats, Shulman and Spritzer (2014) showed that sexual activity did not predict testosterone concentrations in rats exposed to sexually receptive females and that testosterone concentrations only rose on the first day of exposure, but otherwise there was no relationship between sexual behavior and testosterone. In another example, involving golden-collared manikins, the amount of courtship is not directly correlated with the concentration of circulating testosterone although androgens modulate the early courtship experience. Furthermore, territorial aggression was completely uncoupled from testosterone (Fusani et al. 2007; Day et al. 2007).

Because hormones can mediate behavior on a relatively short time scale (Oliviera 2009), I predicted that testosterone and $11-\mathrm{KT}$ should be higher under the low light levels to drive the higher courtship and aggressive behavior under low light, and cortisol should be higher in high light environments in order to mediate conspicuous behavior under varying light conditions and the suspected stress of a higher predation environment. Surprisingly, we did not find flexibility in hormone release rates across light conditions. It is possible that short-term behavior modifications are not accompanied by detectable changes in circulating hormones. Alternatively, changes in guppy behavior due to environmental conditions may be affected by changes in hormone receptors such as reduced or increased binding affinity rather than in an increase in the 
hormone release rates (Adkins-Regan 2008; see Lynn 2008 for a review of proximate mechanisms). My study design would not be able to detect these types of changes.

I predicted cortisol levels would be higher under the potentially more physiologically stressful high light conditions, but found no support for this prediction. It is possible that high light, although a risky environment for guppies, is not a physiologically stressful situation such that males would activate their cortisol response. Furthermore, reduction in conspicuous behaviors under high risk environments may also reduce the cortisol response. It is not known how long it takes for a guppy fish to mount a cortisol response to an acute stressor such as changes in light environment, and it is not exactly known how quickly changes in cortisol levels are reflected in waterborne hormone collection techniques (Martinez-Porchas et al. 2009). In acute stress it appears that most fish show peak cortisol levels 1 hour after exposure to a stressor and return to basal levels after about 6 hours (Iwama et al. 2006). If this result is true of guppies, my collection methods would not have detected correlations of cortisol to the acute light stressor due to the time between my baseline collection period and the second collection period as the fish would have reached basal levels by my second collection. It is also probable that the prolonged exposure to the light condition during the trial period before my second hormone collection may have been enough time for the guppies to habituate to the chronic stress of the high light condition (see Barton et al. 1987 for example in rainbow trout; Martinez-Porchas et al. 2009).

I found that cortisol release rates were higher when females were absent. This indicates that environments lacking receptive females are stressful. This is an interesting finding, as many studies involving primates suggest that all-male groups often show 
lower cortisol levels than male-female groups with receptive females (Arlet et al. 2009; Cedra-Molina et al. 2012). Antagonistic competitive events when receptive females are present have often been cited as a source of increased stress and cortisol levels in these males (Cedra-Molina et al. 2012). These mammals tend to form small bachelor groups as a regular part of their social interactions so living in these environments is quite natural. In guppies, antagonistic aggressive events may be occurring in the absence of females, for males to establish dominance since the males are introduced into unstable social situations. These aggressive events may be more stressful than competitive events for females in the presence of sexually receptive females. Also this result may suggest that bachelor groups in guppy populations may be unnatural.

In conclusion, I found behavioral flexibility in response to fluctuating light environments consistent with previous studies of guppies from high predation sites but in contrast to the only study involving a low predation site. Furthermore, the response only occurred when sexually receptive females were present (Reynolds et al. 1993; Archard 2009; Chapman et al. 2009). I failed to find a relationship between these behavioral modifications and androgens, however. This study encourages future research utilizing low predation populations of guppies to understand the implications of light environments on behavior, as well as the relationship between behavior and androgens in a larger group of organisms. 


\section{Tables:}

Table 11: Description of the behaviors recorded in the Stable Group Tank observations.

\begin{tabular}{|l|l|}
\hline \multicolumn{1}{|c|}{ Behavior } & \multicolumn{1}{|c|}{ Description } \\
\hline Chase & $\begin{array}{l}\text { One male swims directly towards the other } \\
\text { male rapidly }\end{array}$ \\
\hline Display & $\begin{array}{l}\text { One male performs a display clearly } \\
\text { directed at the other male; this display } \\
\text { closely resembles the characteristic } \\
\text { "sigmoid" courtship display of guppies }\end{array}$ \\
\hline Bite & $\begin{array}{l}\text { One male nips at/bites the other male } \\
\text { without chasing him first }\end{array}$ \\
\hline Face-off & $\begin{array}{l}\text { The two males face each other and hover or } \\
\text { swim in a parallel line }\end{array}$ \\
\hline Scuffle & $\begin{array}{l}\text { The two males are parallel to each other } \\
\text { with fins splayed and begin to exhibit a } \\
\text { vibrating motion }\end{array}$ \\
\hline
\end{tabular}


Table 12: Description of the male behaviors recorded in the Challenge Situation Tank Observations.

\begin{tabular}{|c|c|}
\hline Behaviors & Description \\
\hline follow & $\begin{array}{l}\text { Male follows one or both of the females } \\
\text { around the tank }\end{array}$ \\
\hline nip & Male nips/bites at one of the females \\
\hline courtship display & $\begin{array}{l}\text { Male turns his body into an s shape in } \\
\text { front of the female and begins to quiver }\end{array}$ \\
\hline competition & $\begin{array}{l}\text { Male is following a female and the other } \\
\text { male begins to follow the same female (in } \\
\text { competitions males can sigmoid to females, } \\
\text { nip females, etc...) }\end{array}$ \\
\hline sneaks & $\begin{array}{l}\text { Male, tries to copulate with a female } \\
\text { without courting a female using the } \\
\text { sigmoid display }\end{array}$ \\
\hline swings & Male swings his gondopodium \\
\hline switch & $\begin{array}{l}\text { Male is following one female and then } \\
\text { switches females and begins to follow } \\
\text { another female }\end{array}$ \\
\hline display & $\begin{array}{l}\text { One male performs a display clearly } \\
\text { directed at the other male; this display } \\
\text { closely resembles the characteristic } \\
\text { "sigmoid" courtship display of guppies }\end{array}$ \\
\hline competition/dominance & $\begin{array}{l}\text { The two males are engaged in a } \\
\text { competition but leave the females and } \\
\text { begin to do male only behaviors such as } \\
\text { chase, display, bite. }\end{array}$ \\
\hline chase & $\begin{array}{l}\text { One male swims directly towards the other } \\
\text { male at a rapid pace }\end{array}$ \\
\hline scuffle & $\begin{array}{l}\text { The two males repeatedly chase and bite } \\
\text { each other, but it is not obviously instigated } \\
\text { by a particular male }\end{array}$ \\
\hline bite & $\begin{array}{l}\text { One male nips at/bites the other male } \\
\text { without chasing }\end{array}$ \\
\hline
\end{tabular}




\begin{tabular}{|l|l|}
\hline lateral display & $\begin{array}{l}\text { The two males are parallel to each other } \\
\text { with fins splayed and begin to exhibit a } \\
\text { vibrating motion }\end{array}$ \\
\hline foraging & A male is feeding \\
\hline face-off & $\begin{array}{l}\text { The two males face each other and hover or } \\
\text { swim in a parallel line }\end{array}$ \\
\hline moving & $\begin{array}{l}\text { A male is not following a female, engaging } \\
\text { in activities with the other male, and/or not } \\
\text { feeding. Often characterized by swimming } \\
\text { up and down against the glass of the tank. }\end{array}$ \\
\hline
\end{tabular}


Table 13: Male behavior in Female Absent social environments.

\begin{tabular}{|lcc|}
\hline \multicolumn{1}{|c|}{ Behavior } & F Ratio $(\mathrm{DF})$ & Prob $>$ F \\
\hline Aggression Index & $1.27_{(1,41)}$ & 0.267 \\
Chase Duration & & 0.844 \\
\hline
\end{tabular}

Table 14: Male behavior and Female Response to Male behavior in Female Present social environments. * indicates significant results after Bonferroni correction. ** indicates significance before Bonferroni correction.

\begin{tabular}{|lcc|}
\hline \multicolumn{1}{|c|}{ Behavior } & F Ratio $(\mathrm{DF})$ & Prob $>$ F \\
\hline Courtship Index & $4.86_{(1,42)}$ & $0.033^{* *}$ \\
Competition Index & $4.21_{(1,43)}$ & $0.046^{* *}$ \\
Courtship Display Rate & $3.91_{(1,42)}$ & 0.055 \\
$\begin{array}{l}\text { Female Response to } \\
\text { Courtship }\end{array}$ & $6.17_{(1,41)}$ & $0.017^{*}$ \\
\hline
\end{tabular}


Table 15: Analysis of covariance of population, light level, day, and mass as a function of female absent and female present behavioral indices. $* *$ indicates a significant result before Bonferroni correction. * indicates a significant result after Bonferroni correction.

\begin{tabular}{|c|c|c|c|}
\hline & $\begin{array}{c}\text { F Ratio }_{(\text {df })} \text {; } \\
\text { Prob }>\text { F }\end{array}$ & $\begin{array}{c}\text { F Ratio }_{(d f)} ; \\
\text { Prob }>\text { F }\end{array}$ & $\begin{array}{c}\text { F Ratio }_{(d f)} ; \\
\text { Prob }>\text { F } \\
\text { Female Present } \\
\text { Competition }\end{array}$ \\
\hline Population & $1.87_{(2,40)} ; 0.17$ & $0.99_{2,41)} ; 0.38$ & $5.54_{(2,42)} ; 0.007 *$ \\
\hline Light Level & $1.27_{(1,41)} ; 0.27$ & $\begin{array}{l}4.86_{(1,42)} \\
0.033^{* *}\end{array}$ & $4.21_{(1,43)} ; 0.04 * *$ \\
\hline Day & $0.48_{(1,41)} ; 0.49$ & $0.002_{(1,42)} ; 0.96$ & $0.22_{(1,43)} ; 0.64$ \\
\hline Mass & $0.44_{(1,40)} ; 0.51$ & $3.15_{(1,41)} ; 0.08$ & $0.005_{(1,41)} ; 0.94$ \\
\hline Population*Day & $0.75_{(2,41)} ; 0.48$ & $0.76_{(2,42)} ; 0.47$ & $1.27_{(2,43)} ; 0.29$ \\
\hline Day*Light Level & $0.85_{(1,40)} ; 0.36$ & $0.24_{(1,41)} ; 0.63$ & $0.46_{(1,42)} ; 0.50$ \\
\hline $\begin{array}{c}\text { Population*Light } \\
\text { Level }\end{array}$ & $0.37_{(2,41)} ; 0.69$ & $0.02_{(2,42)} ; 0.98$ & $2.58_{(2,43)} ; 0.09$ \\
\hline $\begin{array}{c}\text { Population*Light } \\
\text { Level*Day }\end{array}$ & $0.92_{(2,40)} ; 0.40$ & $0.02_{(2,41)} ; 0.98$ & $0.51_{(2,42)} ; 0.60$ \\
\hline
\end{tabular}


Figures:

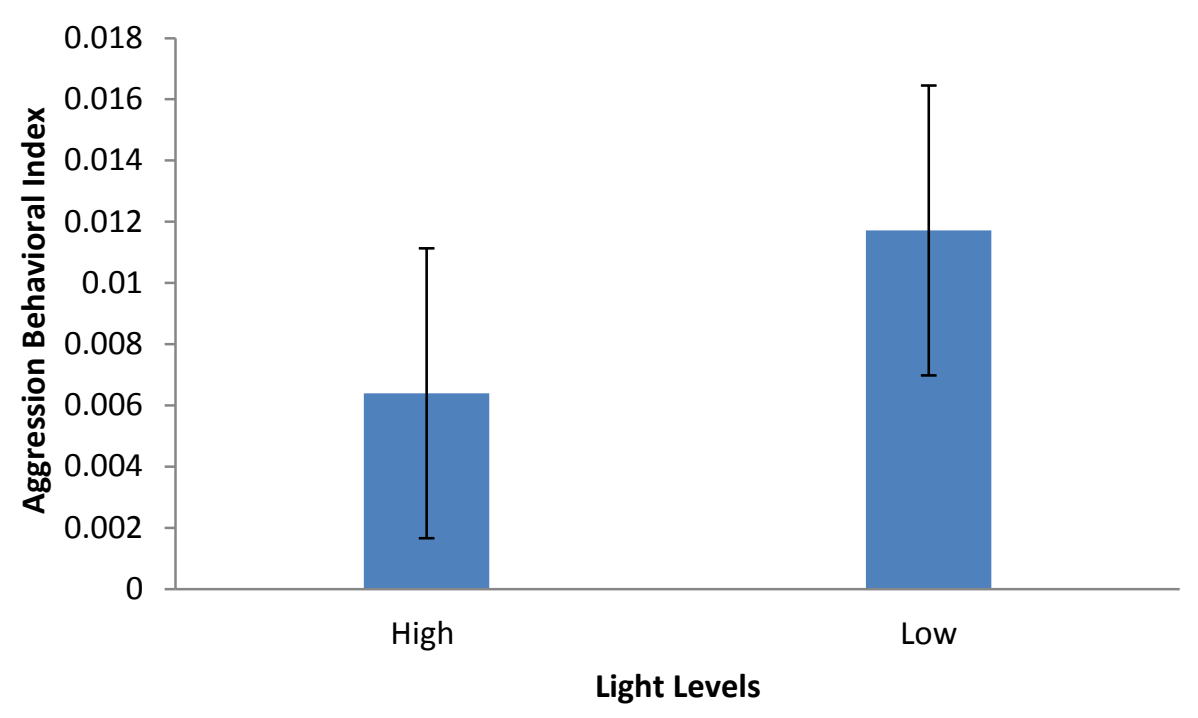

Figure 10: Mean male aggressive behavioral index (+/- standard error) in Female Absent social situations $(n=48)$. 


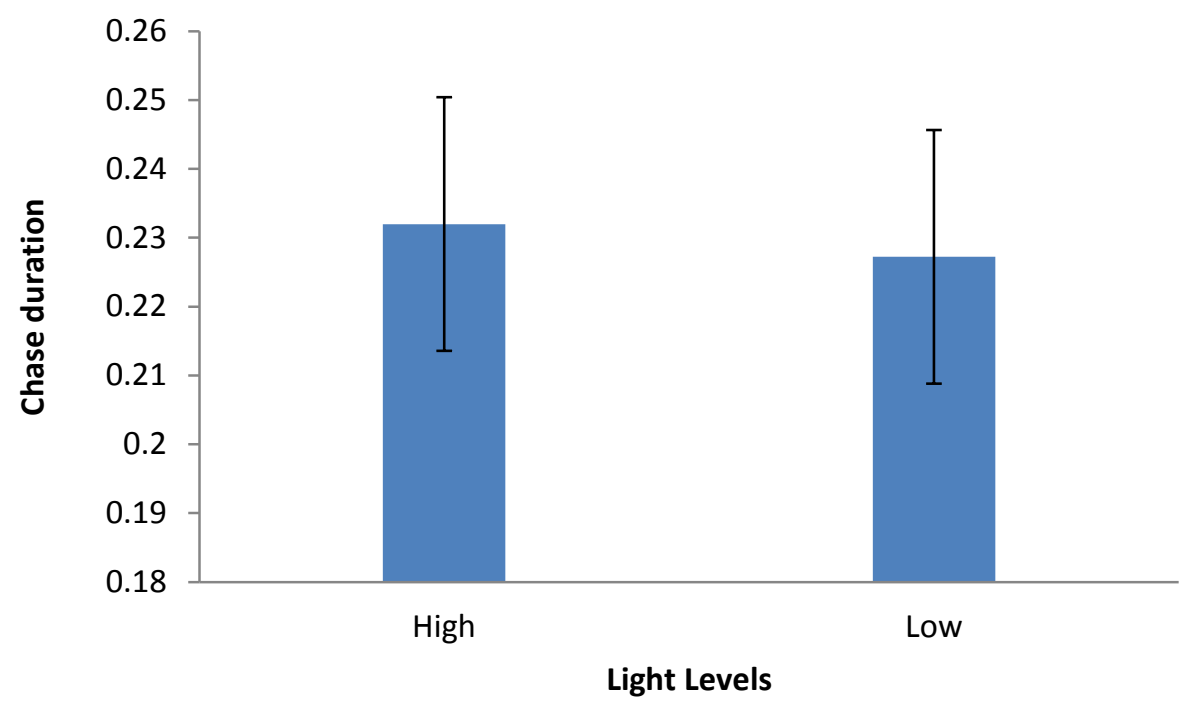

Figure 11: Mean male-male chases performed (+/- standard error) in Female Absent social situations $(\mathrm{n}=48)$. 


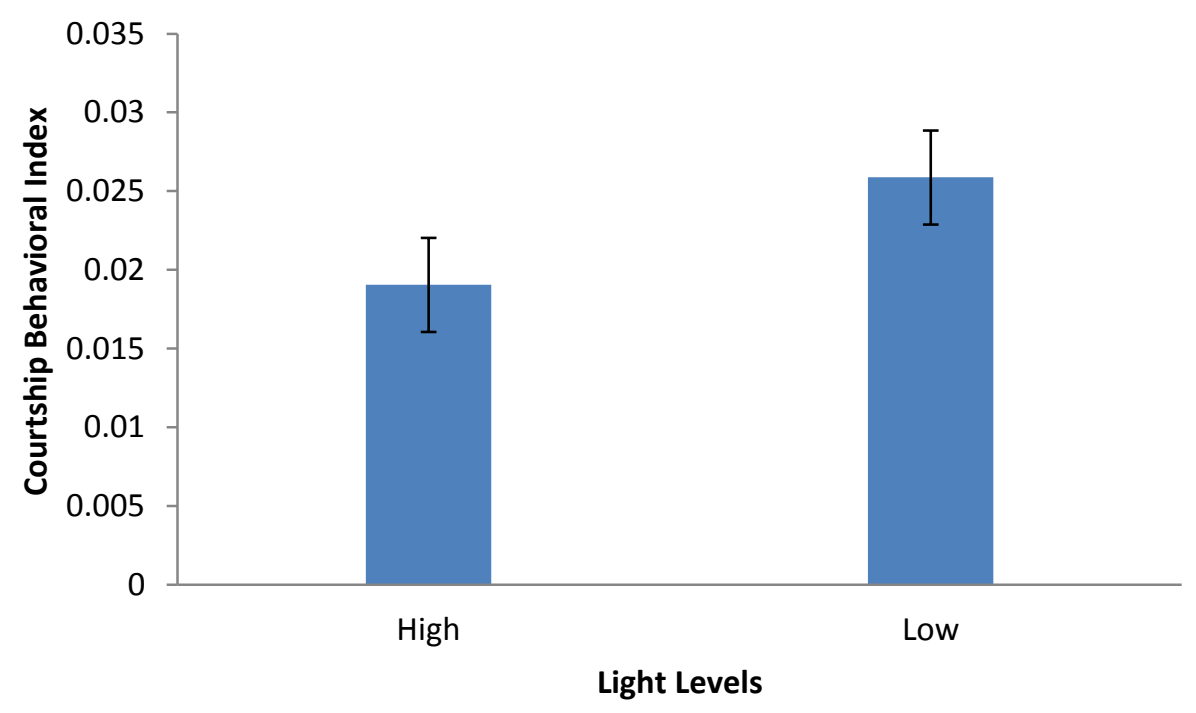

Figure 12: Mean male courtship behavioral index (+/- standard error) in Female Present social situations $(\mathrm{n}=48)$. 


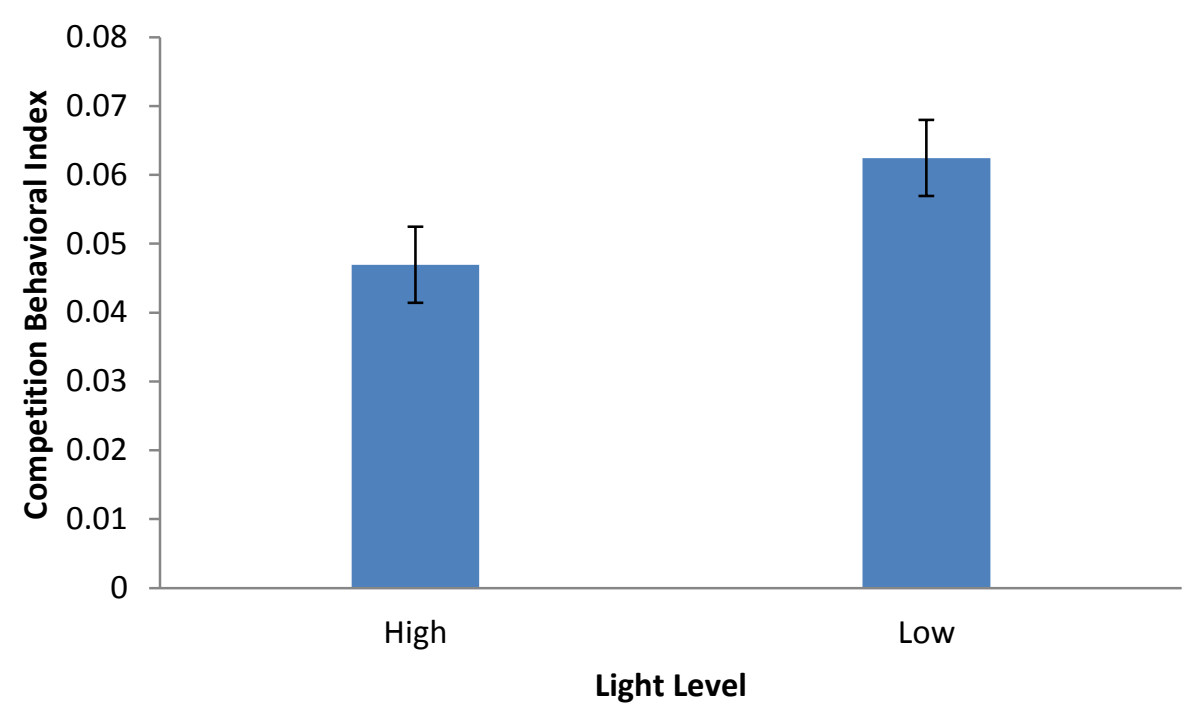

Figure 13: Mean male competition behavioral index (+/- standard error) in Female Present social situations $(n=48)$. 


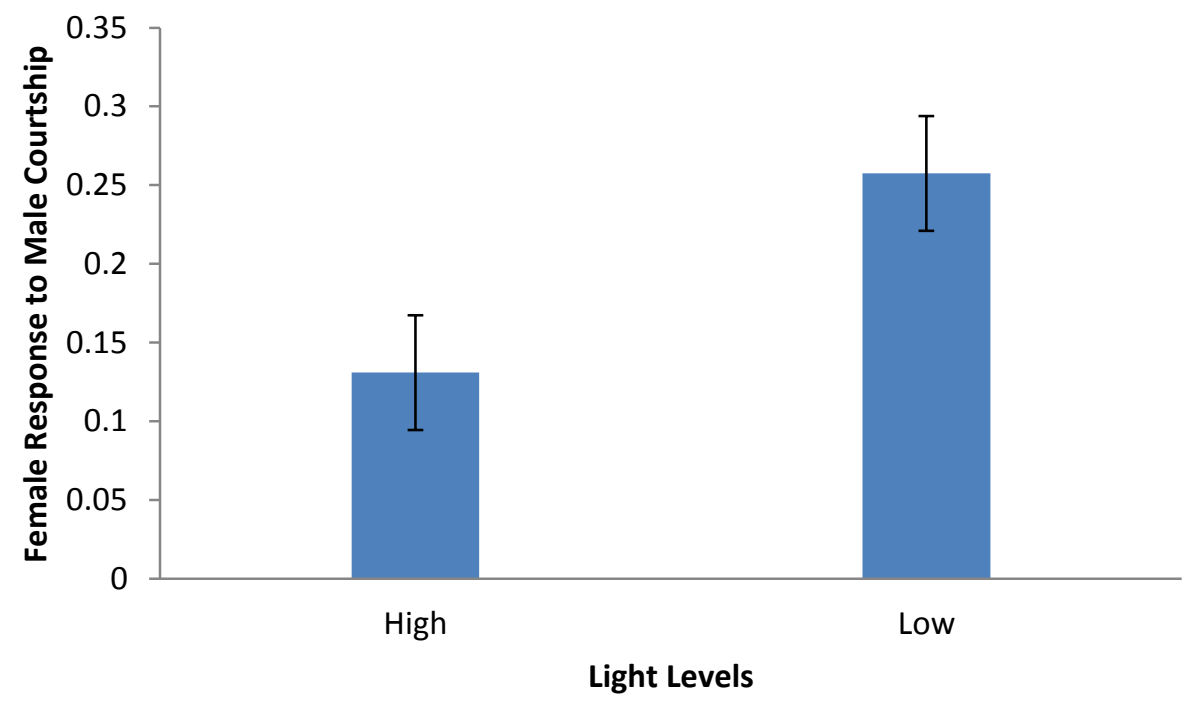

Figure 14: Mean female response to male courtship (+/- standard error) in Female Present social situations $(\mathrm{n}=48)$. 


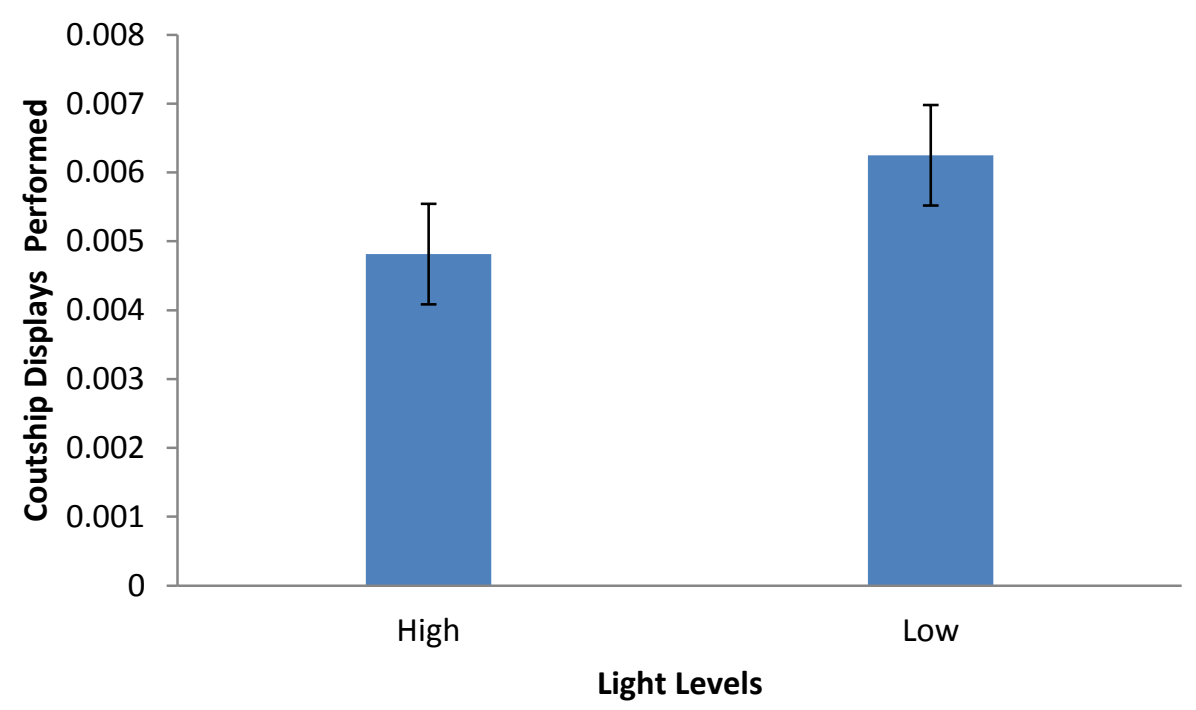

Figure 15: Mean sigmoid rates of males (+/- standard error) in Female Present social situations. 


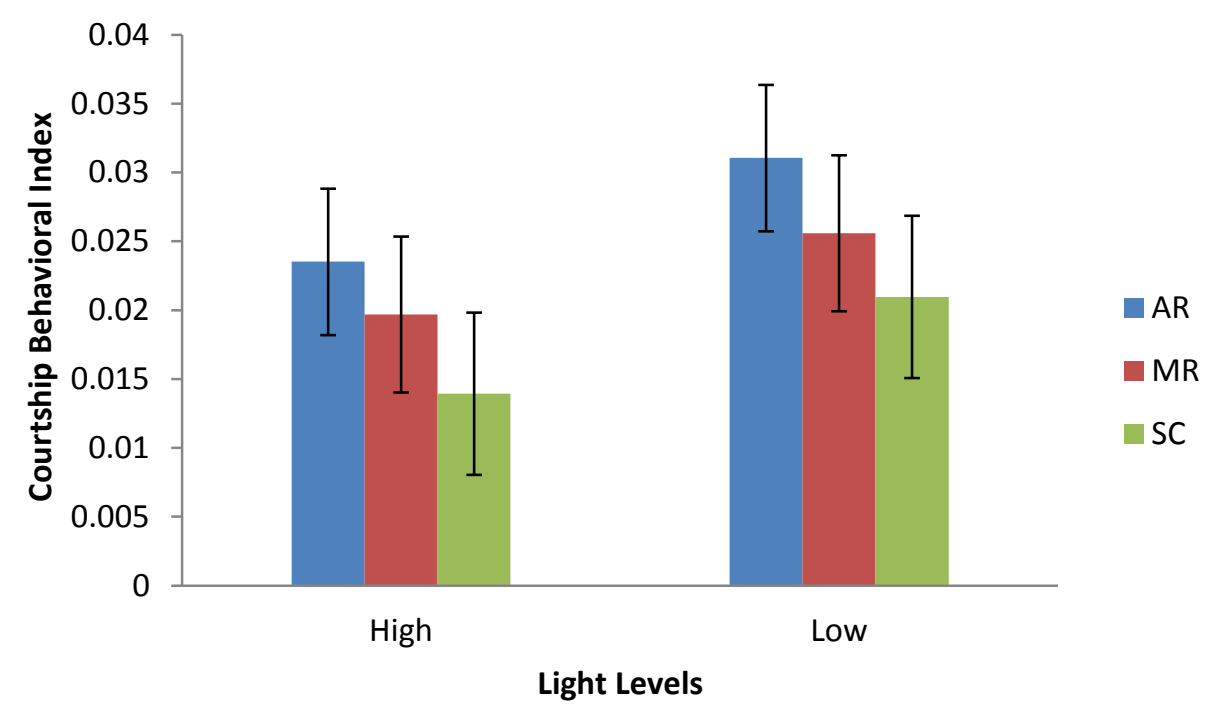

Figure 16: Mean male courtship behavioral index (+/- standard error) in Female Present social situations by population (AR: Aripo River; MR: Marianne River; SC: Small Crayfish). 


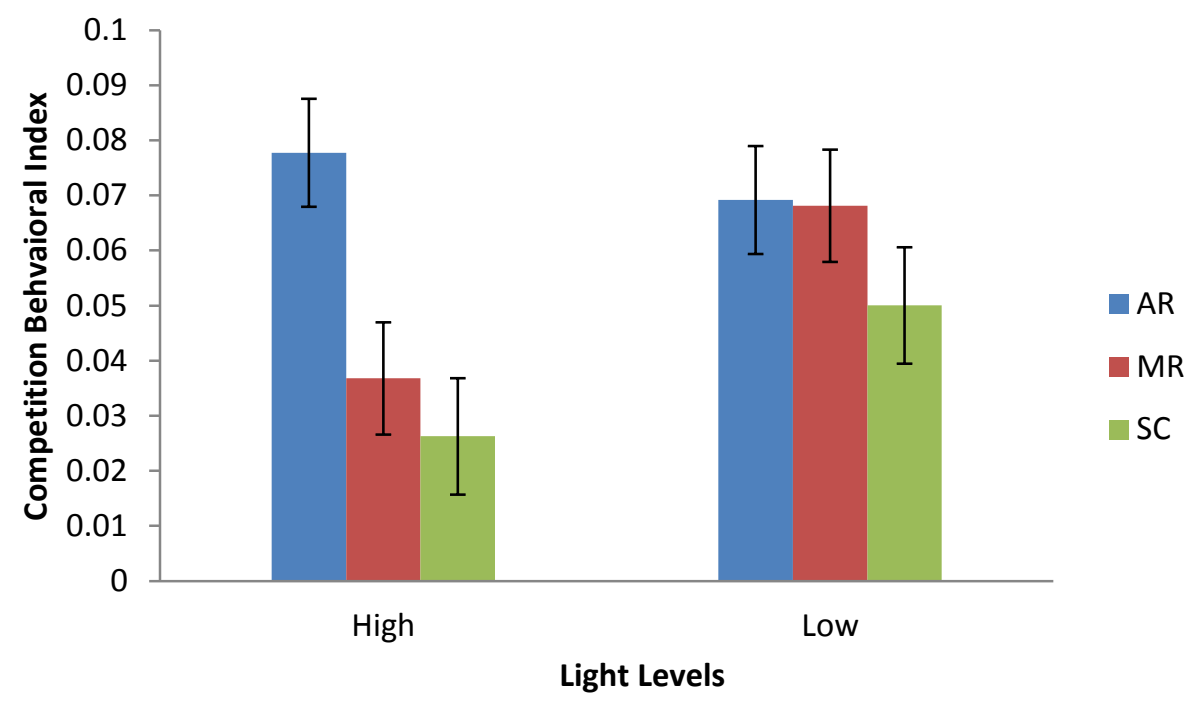

Figure 17: Mean male competition behavioral index (+/- standard error) in Female Present social situations by population (AR: Aripo River; MR: Marianne River; SC: Small Crayfish). 


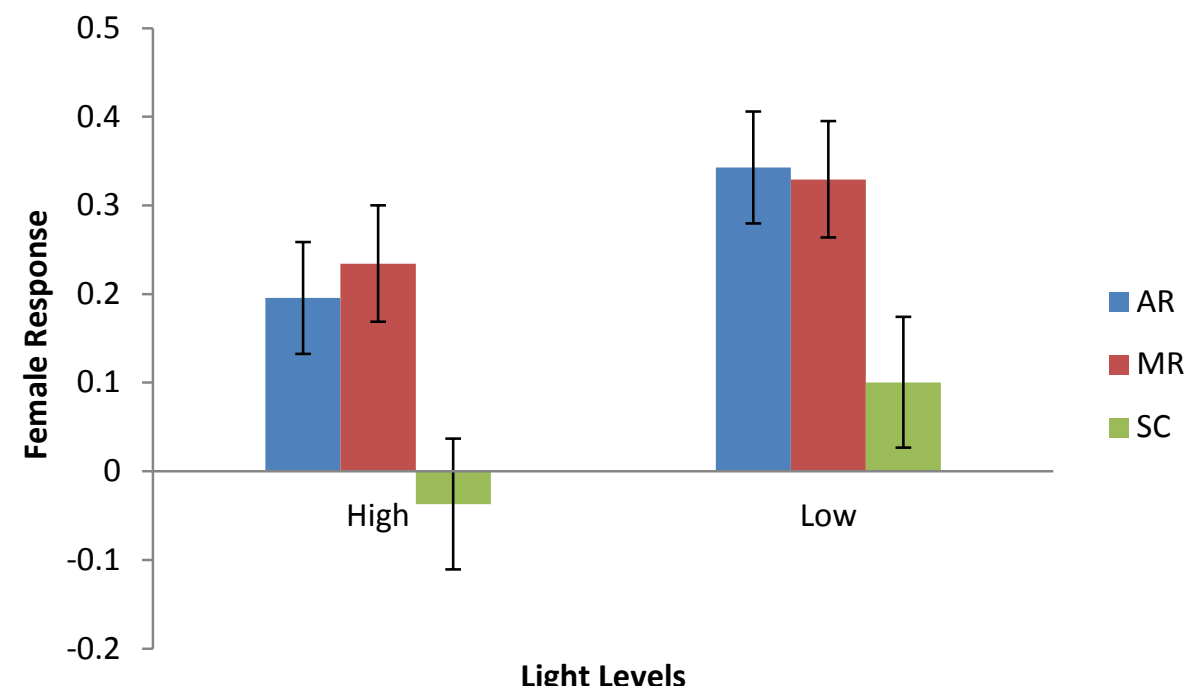

Figure 18: Mean female response to male courtship (+/- standard error) in Female Present social situations by population (AR: Aripo River; MR: Marianne River; SC: Small Crayfish). 


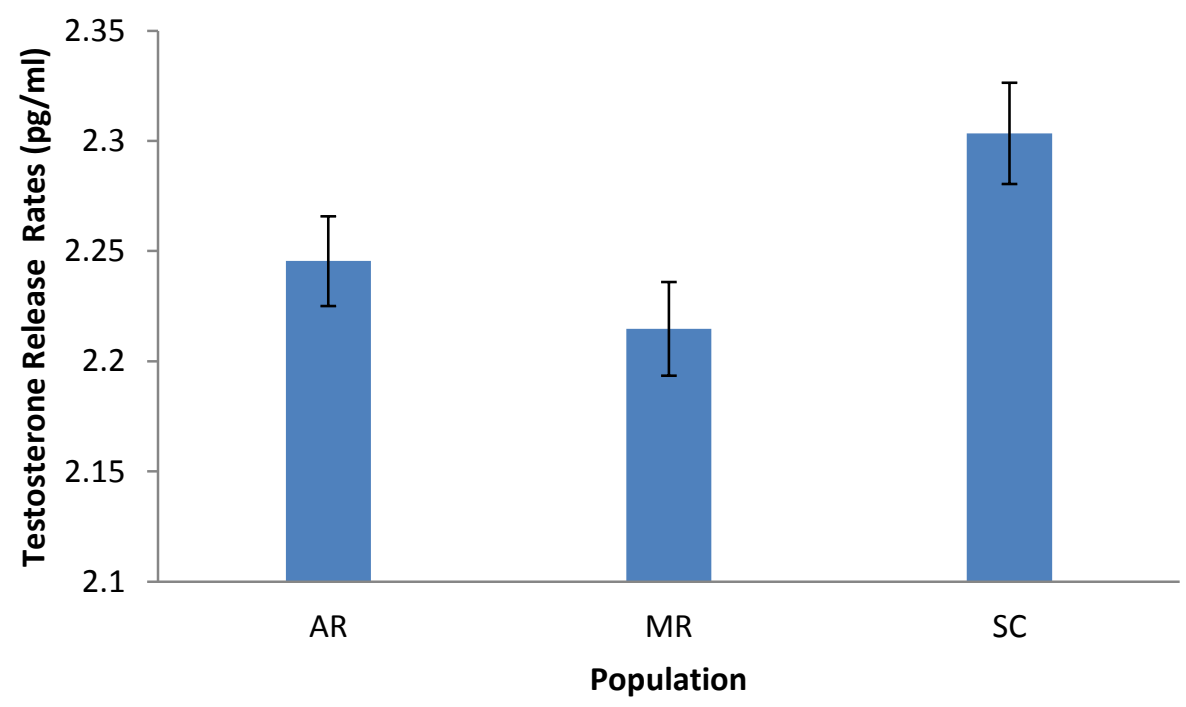

Figure 19: Mean testosterone release rates $(\mathrm{pg} / \mathrm{ml})(+/$ - standard error) by population (AR: Aripo River ( $\mathrm{n}=36)$; MR: Marianne River ( $\mathrm{n}=36)$; SC: Small Crayfish River $(n=36))$. 


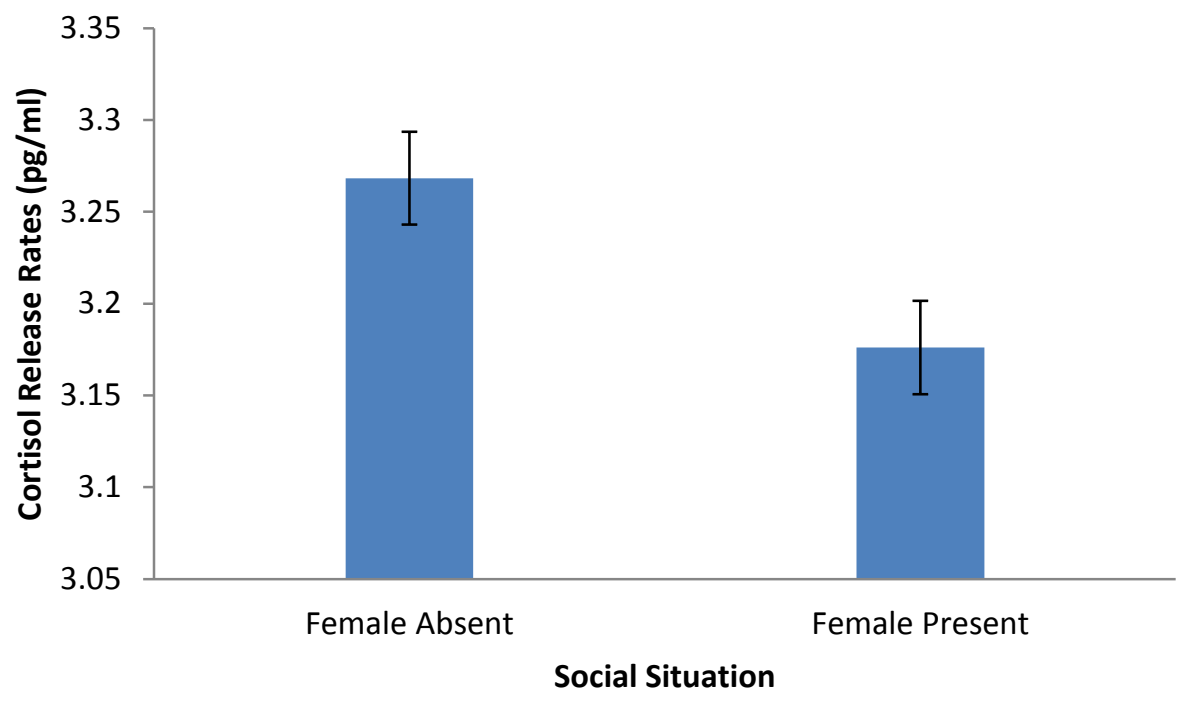

Figure 20: Mean cortisol release rates $(\mathrm{pg} / \mathrm{ml})(+/$ - standard error) in two different social situations (Female Absent $(\mathrm{n}=48)$ and Female Present $(\mathrm{n}=48)$ ). 


\section{BIBLIOGRAPHY}

Adkins-Regan, E. (2008). Do hormonal control systems produce evolutionary inertia? Philosophical Transactions of the Royal Society B, 363, 1599 -1609.

Alcock, J. (2005). Animal Behavior: An Evolutionary Approach ( $8^{\text {th }}$ edition) Sunderland, MA : Sinauer Associates

Archard, G.A., I.C. Cuthill, \& J.C. Partridge. (2009). Light environment and mating behavior in Trinidadian guppies (Poecilia reticulata). Behavioral Ecology and Sociobiology, 64(2), $169-182$.

Arlet, M.E., Grote, M.N., Molleman, F., Isbell, L.A., \& Carey, J.R. (2009). Reproductive tactics influence cortisol levels in individual male gray-cheeked mangabeys (Lophocebus albigena). Hormones and Behavior, 55(1), 210 - 216.

Baerands, G.P., Bower, R., \&Waterbolk, H.T. (1955). Ethological studies on Lebistes reticulates (Peters). An analysis of the male courtship pattern. Behaviour, 8, 249 335.

Barrett, L.T, Evans JP,\& Gasparini C. (2014). The effects of perceived mating opportunities on patterns of reproductive investment by male guppies. PLoS One. 9(4),

Barton, B.A., Schreck, C.B., \& Barton, L.D.(1987). Effects of chronic cortisol administration and daily acute stress on growth, physiological conditions, and stress responses in juvenile rainbow trout. Diseases of Aquatic Organisms, 2, $173-185$. 
Becher, S.A.\& Magurran, A.E. (2000). Gene flow in Trinidadian guppies. Journal of Fish Biology, 56, 241 - 249.

Becker, A., Whitfield, A. K., Cowley, P. D., Järnegren, J., \& Næsje, T. F. (2013). Potential effects of artificial light associated with anthropogenic infrastructure on the abundance and foraging behaviour of estuary-associated fishes. Journal of Applied Ecology, 50, 43 - 50.

Bell, A. M. (2005). Behavioural differences between individuals and two populations of sticklebacks (Gasterosteus aculeatus). Journal of Evolutionary Biology, 18, 464 473.

Bell, A. M. \& Sih, A. 2007. Exposure to predation generates personality in threespined sticklebacks (Gasterosteus aculeatus). Ecology Letters, 10, 828-834.

Bell, A. M. \& Stamps, J. A. (2004). Development of behavioural differences between individuals and populations of sticklebacks, Gasterosteus aculeatus. Animal Behaviour, 68, $1339-1348$.

Blumstein, D.T.,\& Fernandez-Juricic, E. (2004). The emergence of conservation behavior. Conservation Biology, 18, 1175 - 1177.

Borg. B. (1987). Stimulation of reproductive behaviour by aromatizable and nonaromatizable androgens in the male three-spined stickleback, Gasterosteus aculeatus L. In Kullander, S. O. K. \& Fernholm, B., (Eds.), Proceedings of the 5th Congress of European Ichthyologists ( pp. 269-271). Stockholm: Swedish Museum of Natural History. 
Borges, R.A., Oliveira, R.F., Almada, V.C., \& Canario, A.V.M. (1998). Short-term social modulation of 11-ketotestosterone urinary levels in males of the cichlid fish Oreochromis mossambicus during male-female interaction. Acta Ethol., 1, 43 48.

Brooks, R., \& Endler, J.A. (2001). Female guppies agree to differ: Phenotypic and genetic variation in mate-choice behavior and the consequences for sexual selection. Evolution, 55, 1644 - 1655.

Brown, C., Gardner, C. \& Braithwaite, V.A., (2005a). Differential stress responses in fish from areas of high and low-predation pressure. Journal of Comparative Physiology, 175, $305-312$.

Brown C., Jones F., and Braithwaite V. (2005b). In situ examination of boldness-shyness traits in the tropical poeciliid, Brachyraphis episcopi. Animal Behaviour, 70, 1003 $-1009$.

Brown, C., Burgess, F., \& Braithwaite, V. (2007). Heritable and experiential effects on boldness in a tropical poeciliid. Behavioral Ecology and Sociobiology, 62, 237 243.

Buchholz, R. (2007). Behavioral biology: an effective and relevant conservation tool. Trends in Ecology and Evolution, 22(8), 401 - 407.

Budaev, S. V. (1997). "Personality" in the guppy (Poecilia reticulata): a correlational study of exploratory behavior and social tendency. Journal of Comparative Psychology, 111, 399-411. 
Cerda-Molina, A.L., Hernandez-Lopez, L., Diaz-Diaz, G., Mejia-Varas, F., Chavira, R., \& Mondragon-Ceballos, R. (2012). Housing with females increases testosterone and cortisol levels in captive groups of black-handed spider monkeys (Ateles geoffroyi). Zoo Biology, 31(4), 490-497.

Chapman, B. B., Morrell, L.J., \& Krause, J. (2009). Plasticity in male courtship behaviour as a function of light intensity in guppies. Behavioral Ecology and Sociobiology, 63(12), 1757-1763.

Conrad, J.L., Weinersmith, K.L., Brodin, T., Saltz, J.B., \& Sih, A. (2011). Behavioural syndromes in fishes: a review with implications for ecology and fisheries management. Journal of Fish Biology, 78, 395-435.

Cote, J., Fogarty, S., Weinersmith, K., Brodin, T. \& Sih, A. (2010). Personality traits and dispersal tendency in the invasive mosquitofish (Gambusia affinis). Proceedings of the Royal Society B, 277, 1571-1579.

Crispo, E, Bentzen, P., Reznick, D.N, Kinnison, M.T., \& Hendry, A.P. (2006). The relative influence of natural selection and geography on gene flow in guppies. Molecular Ecology, 15, 49-62.

Croft, D.P., Albanese, B., Arrowsmith, B.J., Botham, M.,Webster, M., \& Krause, J. (2003a). Sex-biased movement in the guppy (Poecilia reticulata).Oecologia. 134, $62-68$. 
Croft, D.P., Arrowsmith, B.J., Biebly, J., Skinner, K., White, E.,Couzin, I.D., Magurran, A.E. Ramnarine, I.,\& Krause, J.. (2003b). Mechanisms underlying shoal composition in the Trinidadian guppy, Poecilia reticulata. Oikos. 100, 429-438.

Croft, D. P., Krause, J., Darden, S. K., Ramnarine, I. W., Faria, J. J. \& James, R.. (2009). Behavioural trait assortment in a social network: patterns and implications. Behavior Ecology and Sociobiology, 63, 1495-1503.

Croft, D.P., James, R., Thomas, P.O.R., Hathaway, C., Mawdsley, D., Laland, K.N., \& Krause, J. (2006). Social structure and -co-operative interactions in a wild population of guppies (Poecilia reticulata). Behavioral Ecology and Sociobiology, 56, 644-650.

Crow, R.T., \& Liley, N.R. (1979). A sexual pheromone in the guppy, Poecilia reticulata (Peters). Canadian Journal of Zoology, 57, 184 - 188.

Cureton, J.C., Martin, R.E., \& Deaton, R. (2010). Sex ratio and density alter male mating strategies in the coercive livebearing Mosquitofish. Behaviour, 147(11), 14311442.

Damassa D.A., Smith E.R., Tennent B., \& Davidson J.M. (1977). The relationship between circulating testosterone levels and male sexual behavior in rats. Horm Behav, .8, 275-86.

Day, L.B., Fusani L., Hernandez E., Billo, T.J., Sheldon K.S., Wise, P.M., \& Schlinger, B.A. (2007). Testosterone and its effects on courtship in golden-collared manikins 
(Manacus vitellinus): Seasonal, sex, and age differences. Hormones and Behavior, 51, 69-76.

De Serrano, A.R., Weadick C.J., Price A. C., \& Rodd F.H. (2012). Seeing orange: prawns tap into a pre-existing sensory bias of the Trinidadian guppy. Proceedings of the Royal Society B, 279, 3321 - 3328.

Dill, L.M., Hedrick, A.V., \& Fraser, A. (1999). Male mating strategies under predation risk: do females call the shots. Behavioral Ecology, 10, 452 - 461.

Dingemanse, N. J., Wright, J., Kazem, A. J. N., Thomas, D. K., Hickling, R., \& Dawnay, N. (2007). Behavioural syndromes differ predictably between 12 populations of three-spined sticklebacks. Journal of Animal Ecology, 76, 1128 - 1138.

Drent, P. J., van Oers, K., \& van Noordwijk, A. J. (2003). Realised heritability of personalities in the great tit (Parus major). Proceedings of the Royal Society B, $270,45-51$.

Dugatkin L.A., \& Godin .J.G.J. (1992). Prey approaching predators: a cost-benefit perspective. Ann Zool Fennici, 29, 233 - 252.

Dzieweczynski, T.L., Eklund A.C.,\& Rowland W.J. (2006). Male11-ketotestosterone levels change as a result of being watched in Siamese fighting fish, Betta splendens. Gen Comp Endocrinol, 147, 184 - 9.

Earley, R.L., \& Hsu, Y. (2008). Reciprocity between endocrine state and contest behavior in the killifish, Kryptolebias marmoratus. Hormones \& Behavior, 53, 442 - 451. 
Earley, R. L., Edwards, J. T., Aseem, O., Felton, K., Blumer, L. S., Karom, M., \& Grober, M. S.. (2006). Social interactions tune aggression and stress responsiveness in a territorial cichlid fish (Archocentrus nigrofasciatus). Physiol. Behav., 88, 353 - 363.

Ellis, T., James, J. D., Stewart, C. \& Scott, A. P.. (2004). A non-invasive stress assay based upon measurement of free cortisol released into the water by rainbow trout. J. Fish Biol., 65, 1233 - 1252

Elvidge, C.K., Ramnarine, I., \&Brown, G.E.. (2014). Compensatory foraging in Trinidadian guppies: Effects of acute and chronic predation threats. Current Zoology, 60 (3), 323 - 332.

Endler, J.A. (1978). A predator's view of animal colour patterns. Evolutionary Biology, $11,319-364$.

Endler, J.A. (1987). Predation, light intensity and courtship behavior in Poecilia reticulata (Pisces: Poeciliidae). Animal Behavior, 35, 1376 - 1385.

Endler, J.A. (1995). Multiple-trait coevolution and environmental gradients in guppies. Trends in Ecology and Evolution, 10, 22 - 29.

Endler, J.A., \& Houde, A.E.. (1995). Geographic variation in female preference for male traits in Poecilia reticulata. Evolution, 49, 456 - 468

Endler, J.A .(1991). Variation in the appearance of guppy color patterns to guppies and their predators under different visual conditions. Vis Res, 31, $587-608$. 
Evans J. P., Kelley J. L., Bisazza A., Finazzo E., \& Pilastro A. (2004). Sire attractiveness influences offspring performance in guppies. Proc. R. Soc. Lond. B, 271, 2035 2042.

Fajen, A., \& Breden F. (1992). Mitochondrial DNA sequence variation among natural populations of the Trinidad guppy, Poecilia reticulate. Evolution, 46, 1457- 1465.

Farr, J. (1977). Male rarity or novelty, female choice behavior, and sexual selection in the guppy, Poecilia reticulata Peters (Pisces: Poecilidae). Evolution, 31, 162 -168.

Ferrari, M.C.O., Sih, A.,\& Chivers, D.P. (2009). The paradox of risk allocation: a review and prospectus Animal Behaviour, 78 (3), 579 - 585.

Filby, A.L., Paull, G.C., Hickmore, T.F., \& Tyler, C.R..(2010). Unravelling the neurophysiological basis of aggression in a fish model. BMC Genomics, 11, 498.

Folstad I., \& Karter A.J.. (1992). Parasites, Bright Males, and the Immunocompetence Handicap. The American Naturalist, 139(3), 603-622.

Fusani, L., Day, L.B., Canoine, V., Reinemann, D., Hernandez, E., \& Schlinger, B.A.. (2007). Androgen and the elaborate courtship behavior of a tropical lekking bird. Hormones and Behavior, 51(1), 62-68.

Fusani L., Day L.B., Canoine V., Reinemann D., Hernandez E., \& Schlinger B.A.. (2007). Androgen and the elaborate courtship behavior of a tropical lekking bird. Hormones and Behavior, 51(1),62-68. 
Fuzzen, M.L., Bernier, N.J., \& Van Der Kraak, G.. (2011). Differential effects of $17\{$ beta $\}$-estradiol and 11-ketotestosterone on the endocrine stress response in zebrafish (Danio rerio). Gen. Comp. Endocrinol., 170(2), 365-373.

Gabor, C.R., \& Grober, M.S.. (2010). A potential role of male and female androgen in species recognition in a unisexual-bisexual mating complex. Horm Behav , 57(45), 427-33.

Gamble, S., Lindholm, A. K., Endler, J. A., \& Brooks, R. (2003). Environmental variation and the maintenance of polymorphism: the effect of ambient light spectrum on mating behaviour and sexual selection in guppies. Ecology Letters, $6,463-472$.

Gandolfi, G. (1971). Sexual selection in relation to the social status of males in Poecilia reticulata (Teleostei: Poeciliidae). Boll Zool., 38, 35-48.

Ghalambor, C.K., Angeloni, L.M., \& Carroll, S.P.. (2010). Behavior as Phenotypic Plasticity. In D.E. Westneat and C. W. Fox (Eds). Evolutionary Behavioral Ecology (pgs 90 - 214). London: Oxford University Press.

Ghalambor, C. K., Walker, J. A., \& D. N. Reznick.. (2003). Multi-trait selection, adaptation, and constraints on the evolution of burst swimming performance. Integrative and Comparative Biology, 43, 431-438.

Ghalambor, C.K., McKay J. K., Carroll S.P.,\& Reznick D.N. (2007). Adaptive versus non-adaptive phenotypic plasticity and the potential for contemporary adaptation in new Environments. Functional Ecology, 21, 394 -407. 
Godin, J.G. (1995). Predation risk and alternative mating tactics in male Trinidadian guppies (Poecilia reticulata). Oecologia, 103, 224-229.

Godin, J.G.J., \& Briggs S.E. (1996). Female mate choice under predation risk in the guppy. Animal Behaviour, 51 (1), 117-130.

Godin, J. G. J., \& Dugatkin, L. A.. (1996). Female mating preference for bold males in the guppy, Poecilia reticulata. Proceedings of the National Academy of Sciences, 93, 10262-10267.

Gong, A. (1997). The effects of predator exposure on the female choice of guppies (Poecilia reticulata) from a high-predation population. Behaviour, 134 (5/6), 373 389.

Borg, B. (1994). Androgens in teleost fishes. Comp. Biochem. Physiol., 109 (3), 219245.

Gong A., \& Gibson R.M. (1996). Reversal of a female preference after visual exposure to a predator in the guppy, Poecilia reticulata. Animal Behavior, 52, 1007-1015.

Goodwin, T.W. (1984). The biochemistry of carotenoids. New York, NY: Chemical Publishing Co, Inc.

Gorlick, D.L. (1976). Dominance hierarchies and factors influencing dominance in the guppy Poecilia reticulata (Peters). Animal Behaviour, 24, 336 - 346.

Gosling, S. D. (2001). From mice to men: what can we learn about personality from animal research? Psychological Bulletin, 127, 45-86. 
Grether, G.F. (2000). Carotenoid limitation and mate preference evolution: a test of the indicator hypothesis in guppies (Poecilia reticulata). Evolution, 54, 1712 - 1724.

Grether, G.F., Millie, D.F., Bryant, M.J., Reznick, D.N., \& Mayea, W. (2001). Rain forest canopy cover, resource availability, and life history evolution in guppies. Ecology, 82(6), 1546 - 1559.

Grether G.F., Kasahara S., Kolluru G.R., \& Cooper E.L. (2004). Sex-specific effects of carotenoid intake on the immunological response to allografts in guppies (Poecilia reticulata). Proc. R. Soc. B., 271, $45-49$.

Grether G.F., Hudon J., \& Millie D.F.(1999). Carotenoid limitation of sexual coloration along an environmental gradient in guppies. Proc R Soc Lond B Biol Sci., 266, $1317-1322$.

Hallgren S.L.E., Linderoth M., \& Olsen K.H. (2006). Inhibition of cytochrome brain aromatase reduces two male specific sexual behaviours in the male Endler guppy (Poecilia reticulata). General and Comparative Endocrinology, 147, 323 - 328.

Hamilton W.D., \& Zuk M. (1982). Heritable true fitness and bright birds: a role for parasites? Science, 218, $384-387$.

Hannes, R.P. (1984). Androgen and corticoid levels in blood and body extracts of high and low ranking swordtail males (Xiphophorus helleri) before and after social isolation. Z. Tierpsychol. $66,70-76$. 
Hannes, R.P.. (1986). Blood and whole-body androgen levels of male swordtails correlated with aggression measures in a standard-opponent test. Aggress. Behav., $12,249-254$.

Haskins, C.P., and E.F. Haskins. (1950). Factors affecting sexual selection as an isolating mechanism in the poeciliid fish, Lebistes reticulates. Proceedings of the National Academy of Sciences USA, 36, 464 - 476.

Haskins, C.P., Haskins, E.F., McLaughlin, J.J.A., \& Hewitt, R.E. (1961). Polymorphism and population structure in an ecological study. In W.F. Blair (Ed.) Vertebrate speciation (pp.320-395). Austin, TX: University of Texas Press.

Helfman, G.S. (1993). Fish behaviour by day, night and twilight. In T.J. Pitcher (Ed.) Behaviour of teleost fishes ( $2^{\text {nd }}$ ed) (pp. 479-512). London, England: Chapman \& Hall.

Hibler T.L., \& Houde A.E. (2006). The effect of visual obstructions on the sexual behaviour of guppies: the importance of privacy. Animal Behaviour, 72, $959-$ 964.

Hirschenhauser K, \& Oliveira RF.(2006). Social modulation of androgen levels in Vertebrates: a meta-analysis of the challenge hypothesis. Animal Behavior, 71, $265-77$.

Hoar, W.S. (1962). Reproductive behavior of fish. Gen comp. Endocr., 1, 206 -216.

Houde, A.E. (1988). Genetic difference in female choice between two guppy populations. Animal Behavior, 36, 510 - 516 
Houde, A.E. (1997). Sex, color, and mate choice in guppies. Princeton: Princeton University Press

Houde, A.E, \& Torio, A. (1992). Effect of parasitic infection on male colour pattern and female choice in guppies. Behavioral Ecology, 3, 346 - 351.

Hughes, K.A., Du, L., Rodd, H., \& Reznik, D. (1999). Familiarity leads to female mate preference for novel males in the guppy, Poecilia reticulate. Animal Behaviour, 58, $907-916$.

Huntingford, F. A.. (1976). The relationship between anti-predator behaviour and aggression among conspecifics in the three-spined stickleback, Gasterosteus aculeatus. Animal Behaviour, 24, 245 - 260.

Iwama, G.K., Afonso, L.O.B., \& Vijayan, M.M.. (2006). Stress in fishes. In D.H. Evans \& J.B. Claiborne (Eds.), The physiology of fishes ( $3^{\text {rd }}$ ed.) (pp. 319- 342). :Taylor and Francis

Jirotkul, M. (1999a). Population density influences male-male competition in guppies. Animal Behaviour, 58, 1169 - 1175.

Jirotkul, M. (1999b). Operational sex ratio influences female preference and male-male competition in guppies. Animal Behaviour, 58, 287 - 294.

Jirotkul, M. (2000). Male trait distribution determined alternative mating tactics in guppies. Journal of Fish Biology, 56, 1427 - 1434.

Johnson, J. C., \& Sih, A.. (2005). Precopulatory sexual cannibalism in fishing spiders 
(Dolomedes triton): a role for behavioral syndromes. Behavior Ecology and Socio-biology, 58, $390-396$.

Jordan L.A., \& Brooks, R.C. (2012). Recent social history alters male courtship preferences. Evolution, 66 (1), 280 - 287.

Kelly C.D., \& Godin G. J.. (2001). Predation risk reduces male-male sexual competition in the Trinidadian guppy (Poecilia reticulata). Behav Ecol Sociobiol., 51, 95 100.

Kelley, J., \& Brown, C. (2011). Predation risk and decision making in poeciliid prey. In J.P Evans, A. Pilastro, \& I. Schlupp, Ecology and Evolution of Poeciliid Fishes (pp 174-184). Chicago, IL:University of Chicago Press.

Kelley, J.L.,Graves, J.A., \& Magurran, A.E. (1999). Familiarity breeds contempt in guppies. Nature, 401, 661 .

Kelley, J.L., Phillips, S.C., \& Evans, J.P. (2013). Individual consistency in exploratory behavior and mating tactics in male guppies. Naturwissenschafter DOI 10.1007/s00, 114-013- 1097-3.

Ketterson, E.D., Atwell, J.W., \& McGlothlin, J.W. (2009). Phenotypic integration and independence: hormones, performance, and response to environmental change. Integrative and Comparative Biology, 49, 365 - 379.

Khoo, G., Lim, K.F., Gan, D., Chen, F., Chan, W., Lim, T.M., \& Phang, V.. (2002). Genetic diversity within and among feral populations and domesticated strains of 
the guppy (Poecilia reticulate) in Singapore. Molecular Biotechnology, 4, 367 378.

Kindler, P.M., Bahr, J.M., \& Philipp, D.P.. (1991). The effects of exogenous 11ketotestosterone, testosterone, and cyproterone acetate onn prespawning and parental care behaviors of male bluegill. Hormones and Behavior, 25(3), 410 423.

Kodric-Brown, A. (1989). Dietary carotenoids and male mating success: An environmental component of female choice. Behav. Ecol. Sociobiol., 25, 393 401.

Kodric-Brown, A. and Brown J.H. (1984). Truth in advertising: The kinds of traits favored by sexual selection. Am. Nat., 124, 305 - 322 .

Kodric-Brown, A. (1989). Dietary carotenoids and male mating success: An environmental component of female choice. Behav. Ecol. Sociobio., 25, 393 401.

Kodric-Brown, A. (1992). Male dominance can enhance mating success in guppies. Animal Behaviour, 44, 165 - 167.

Kolluru, G.R., \& Grether, G.F. (2005). The effects of resource availability on alternative mating tactics in guppies (Poecilia reticulata). Behavioral Ecology, 16, 294 300. 
Kolluru, G.R., Grether, G.F., \& Contreras, H. (2007). Environmental and genetic influences on mating strategies along a replicated food availability gradient in guppies (Poecilia reticulata). Behav Ecol Sociobiol., 61, 689 - 701.

Komers, P. E. (1997). Behavioral plasticity in variable environments. Canadian Journal of Zoology, 75, $161-169$.

Liley, N.R.. (1966). Ethological isolating mechanisms in four sympatric species of Poeciliid fishes. Behaviour (suppl), 13, 1- 197.

Liley N.R.,\& Seghers B.H. (1975). Factors affecting the morphology and behaviour of guppies in Trinidad. In G.P. Baerends, C. Beer, \& A. Manning (Eds.), Function and evolution in behavior (pp 92-118). Oxford: Clarendon Press.

Lima, SL,\& Bednekoff, PA.. (1999). Temporal variation in danger drives antipredator behavior: the predation risk allocation hypothesis. American Naturalist, 153, 649 -659 .

Lima, S.L. (1992). Life if a multi-predator environment: some considerations for antipredatory vigilance. Annales Zoologici Fennici, 29, 217 - 226.

Lima, S.L. (1998). Nonlethal effects in the ecology of predator-prey interactions. BioScience, 48, 25 - 34.

Lindholm, A.K., Breden, F., Alexander, H.J., Chan, W., Thakurta, S.G.,\& Brooks, R.. (2005). Invasion success and genetic diversity of introduced populations of guppies Poecilia reticulata in Australia. Molecular Ecology, 14, 3671 - 3682. 
Lindholm, J.B., Auster, P.J., \& Kaufman, L.S.. (1999). Habitat-mediated survivorship of juvenile (0-year) Atlantic cod. Gadus morhua. Mar Ecol. Prog. Ser., 180, 247255.

Lockard, R.B., \& Owings, D.H.. (1974). Seasonal variation in moonlight avoidance by bannertail kangaroo rats. Journal of Mammalogy, 55, 189 - 193.

Long, K.D., \& Rosenqvist, G. (1998). Changes in male guppy courting distance in response to a fluctuating light environment. Behavioral Ecology and Sociobiology, 44, $77-83$.

Lopez, P., Hawlena, D., Polo, V., Amo, L., \& Martin, J. (2005). Sources of individual shy-bold variations in antipredator behavior of male Iberian rock lizards. Animal Behavior, 69, 1 - 9.

Lorenz, K. (1965). Evolution and modification of behavior. Chicago, IL:University of Chicago Press.

Luyten, P.H., \& Liley, N.R. (1991). Sexual Selection and Competitive Mating Success of Male Guppies (Poecilia reticulata) from four Trinidad populations. Behavioral Ecology and Sociobiology, 28(5), 329 - 336.

López-Sepulcre, A., Gordon, S. P., Paterson, I. G., Bentzen, P., \& Reznick, D. N. (2013). Beyond lifetime reproductive success: the posthumous reproductive dynamics of male Trinidadian guppies. Proc. $R$. Soc. B, 280, $1-8$.

Lorenzi, V., Earley, R. L., Rodgers, E. W., Pepper, D. R., \& Grober, M. S. (2008). Diurnal patterns and sex differences in cortisol, 11-ketotesetorerone, testosterone, 
and 17 beta-estradiol in the bluebanded goby (Lythrypnus dalli). Gen. Comp. Endocr., 155, 438 - 446.

Luyten P.H., \& Liley N.R. (1985). Geographic variation in the sexual behavior of the guppy, Poecilia reticulata (Peters). Behaviour, 95(1/2), 164 - 179.

Lynn, S.E. (2008). Behavioral insensitivity to testosterone: Why and how does testosterone alter paternal and aggressive behavior in some avian species but not others? General and Comparative Endocrinology, 157, 233 - 240.

Magurran, A.E. (1990). The adaptive significance of schooling as an anti-predator defence in fish. Ann. Zoo. Fennici, 27, 51 - 66.

Magurran, A.E.(2005). Evolutionary Ecology:The Trinidadian Guppy. Oxford: Oxford University Press

Magurran, A.E., \& Nowak M.A. (1991). Another battle of the sexes: the consequences of sexual asymmetry in mating costs and predation risk in the guppy, Poecilia reticulata. Proc $R$ Soc Ser B, 246, 31 - 38.

Magurran, A.E., \& Seghers, B.H. (1990). Risk sensitive courtship in the guppy Poecilia reticulata. Behaviour, 112, 194 - 201.

Magurran, A.E., \& Seghers, B.H. (1991).Variation in schooling and aggression amongst guppy (Poecilia reticulata) populations in Trinidad. Behaviour, 118, 214 - 234.

Magurran, A.E., \& Segher, B.H. (1994). Predator inspection behaviour covaries with schooling tendency amongst wild guppy, Poecilia reticulata, populations in Trinidad. Behaviour, 128, $121-134$. 
Martinez-Porchas, M., Martinez-Cordova, L.R., \& Ramos-Enriquez, R. (2009).Cortisol and glucose: Reliable indicators of fish stress? Pan-American Journal of Aquatic Sciences, 4(2), 158 - 178.

Miles, D.B, Sinervo, B., Hazard, L., Svensson, E., \& Costa, D. (2007). Alternative Mating Strategies: Integrating Hormones, Physiology and Behavior. Functional Ecology, 21, $653-665$.

Millar, N.P., Reznick, D.N., Kinnison, M.T. \& Hendry, A.P.. (2006). Disentangling the selective factors that act on male color in wild guppies. Oikos, 113, 112.

Miller L.K.,\& Brooks R. (2005). The effects of genotype, age, and social environment on male ornamentation, mating behavior, and attractiveness. Evolution, 59 (11), 2414 $-2425$.

Moran, N.A. (1992). The evolutionary maintenance of alternative phenotypes. The American Naturalist, 139(5), 971 - 989.

Moyle, P.B., \& Cech, J.J. (2000). Fishes and Introduction to Ichthyology (4th ed). Upper Saddle River, NJ: Prentice Hall

Munz, F.W., \& W.N. McFarland, (1973). The significance of spectral position in the rhodopsins of tropical marine fishes. Vision Res., 13, 1829 - 1874.

Nonacs, P., \& Blumstein D.T.. (2010). Predation Risk and Behavioral Life History. In D.E. Westneat \& C. W. Fox (Eds) Evolutionary Behavioral Ecology (pp. 90 214). London, England: Oxford University Press. 
Oliveira, R.F. (2009). Social behavior in context: Hormonal modulation of behavioral plasticity and social competence. Integrative and Comparative Biology, 49 (4), $423-440$.

Oliveira RF, Almada VC,\& Canario AVM.. (1996). Social modulation of sex steroid concentrations in the urine of male cichlid fish Oreochromis mossambicus (Teleostei: Cichlidae). Horm Behav, 30, 2 - 12.

Oliveira, R.F., Hirschenhauser, K. Carneiro, L.A., Adelino, V.M., \& Canario, A. (2002). Social modulation of androgen levels in male teleost fish. Comparative Biochemistry and Physiology Part B, 132, $203-215$.

Packer, C., Swanson, A., Ikanda, D., \& Kushnir, H.. (2011). Fear of darkness, the full moon and the nocturnal ecology of African lions. PLoS ONE, 6 (7), DOI: 10.1371/journal.pone.0022285.

Parrish, J. K. (1992). Do predators 'shape' fish schools: interactions between predators and their schooling prey. Neth. J. Zool., 42, $358-370$.

Pettersson, L.B., Ramnarine, I.W., Becher, S.A., Mahabir, R., \& Magurran, A.E. (2004). Sex ratio dynamics and fluctuating selection pressures in natural populations of the Trinidadian guppy, Poecilia reticulata. Behavioral Ecology and Sociobiology, $55,461-468$.

Piersma T., \& Drent J. (2003). Phenotypic flexibility and the evolution of organismal design.Trends in Ecology and Evolution, 18, 228 - 233.

Piyapong, C., Krause, J., Chapman, B. B., Ramnarine, I. W., Louca, V. \& Croft, D. P.. 
(2010). Sex matters: a social context to boldness in guppies (Poecilia reticulata).

Behavioral Ecology, 21, 3 - 8 .

Pocklington R.,\& Dill L.M.. (1995). Predation on females or males: who pays for bright male traits? Animal Behavior, 49, 1122 - 1124.

Price, A.C., \& F.H. Rodd.. (2006). The effect of social environment on male-male competition in guppies (Poecilia reticulata). Ethology, 112(1), 22 - 32.

Reale, D., \& Festa-Bianchet, M. (2003). Predator-induced selection on temperament in bighorn ewes. Animal Behaviour, 65, 463 - 470.

Reid, S. G., Bernier, N. J., \& Perry, S. F.. (1998). The adrenergic stress response in fish: control of catecholamine storage and release. Comparative Biochemistry and Physiology Part C , 120, 1 - 27.

Reynolds, J.D.. (1993). Should attractive individuals court more? Theory and a test. American Naturalist, 141, 914 - 927.

Reynolds, J. D., Gross, M.R., \& Coombs, M.J.. (1993). Environmental conditions and male morphology determine alternative mating behavior in Trinidadian guppies. Animal Behavior, 45, 145 - 152.

Reznick, D., Butler, M.J., \& Rodd, H.. (2001). Life-history evolution in guppies. VII. The comparative ecology of high- and low-predation environments. American Naturalist, 157, 126 - 140. 
Reznick, D., \& Bryant, M.. (2007). Comparative long-term mark-recapture studies of guppies (Poecilia reticulate): differences among high and low predation localities in growth and survival. Ann. Zool. Fennici, 44, 152 - 160.

Reznick,D., Butler, M.J., \& Rodd, H.. (2001). Life-History evolution in guppies.VII. The comparative ecology of high - and low-predation environments. The American Naturalist, 157, 126 - 140.

Reznick, D.N., Butler IV, M.J., Rodd, F.H., \& Ross, P.(1996). Life-history evolution in guppies (Poecilia reticulata)6. Differential mortality as a mechanism for natural selection. Evolution, 50(4), 1651 - 1660.

Reznick,D., \& Endler, J.A., (1982). The impact of predation on life history evolution in Trinidadian guppies(Poecilia reticulata). Evolution, 36, 160 - 177.

Reznick, D. N., Shaw, F.H., Rodd, F.H., \& Shaw, R.G.. (1997). Evaluation of the rate of evolution in natural populations of guppies (Poecilia reticulata). Science, 275, $1934-1937$.

Reynolds, J.D., Gross, M.R., \& Coombs, M.J. (1993). Environmental conditions and male morphology determine mating behaviour in Trinidadian guppies. Animal. Behavior, 45, 145-152.

Rodd, F.H., \& Sokolowski, M.B.. (1995). Complex origins of variation in the sexual behaviour of male Trinidadian guppies, Poecilia reticulata: interactions among social environment, heredity, body size and age. Animal Behaviour, 49, 1139 1159. 
Rodd, F.H., \& Reznick, D.N. (1997). Variation in the demography of guppy populations: The importance of predation and life histories. Ecology, 78, $405-418$.

Russel, S. T., Kelley, J.L., Graves, J.A., \& Magurran, A.E. (2004). Kin structure and shoal composition dynamics in the guppy, Poecilia reticulate. Oikos, 106, 520 526.

Ryer, C.H., \& B.L. Olla. (1998). Effect of light on juvenile walleye pollock shoaling and their interaction with predators. Marine Ecology Progress Series, 167, 215 - 226.

Schwartz, A.K. \& Hendry, A.P. (2007). A test for the parallel co-evolution of male colour and female preference in Trinidadian guppies (Poecilia reticulata) Evol. Ecol. Res., 9, $71-90$.

Schwartz, A.K., \& Hendry A.P. (2010). Testing the influence of local forest canopy clearing on phenotypic variation in Trinidadian guppies. Functional Ecology, 24, $354-364$.

Scott, A.P., Hirschenhauser, K., Bender, N., Oliveira, R.., Earley, R.L., Sebire, M., Ellis, T., Pavlidis, M., Hubbard, P.C., Huertas, M., \& Canario, A.. (2008). Non-invasive measurement of steroids in fish-holding water: important considerations when applying the procedure to behaviour studies. Behaviour, 145, 1307 - 1328.

Seghers,B.H.(1974). Schooling behavior in the guppy (Poecilia reticulata): an evolutionary response to predation. Evolution, 28, 486 - 489. 
Shulman L.M., \& Spritzer M.D. (2014). Changes in the sexual behavior and testosterone levels of male rats in response to daily interactions with estrus females. Physiol Behav., 133, 8 - 13.

Sih, A., Bell, AM., Johnson, J.C., \& Ziemba, R.E.. (2004a). Behavioral Syndromes: An Integrative Overview. The Quarterly Review of Biology, 79(3), 241 - 277

Sih, A., Bell, A., \& Johnson, J.C. (2004b). Behavioral syndromes :an ecological and evolutionary overview. Trends in Ecology and Evolution, 19, 372 - 378.

Sih, A., Bell, A., \& Johnson, J.C. (2010). Behavioral syndromes. In D.E. Westneat \& C. W. Fox (Eds.), Evolutionary Behavioral Ecology (pgs 90 - 214). London, England: Oxford University Press.

Sih, A., Ferrari, M. C. O. \& Harris, D. J.. (2011). Evolution and behavioural responses to human-induced rapid environmental change. Evolutionary Applications, 4, 367 387.

Shoji, A., Yokoyama, J., \& Kawata, M.. (2007). Molecular phylogeny and genetic divergence of the introduced populations of Japanese guppies, Poecilia reticulata. Conservation Genetics, 8, 261 - 271.

Smith, B. R., \& Blumstein, D. T. (2010). Behavioral types as predictors of survival in Trinidadian guppies (Poecilia reticulata). Behavioral Ecology, 21, 919 - 926.

Snell-Rood, E.C.. (2013). An overview of the evolutionary causes and consequences of behavioural plasticity. Animal Behaviour, 85(5), 1004 - 1011. 
Templeton, C.N., \& Shriner, W.M. (2004). Multiple selection pressures influence Trinidadian guppy (Poecilia reticulata) antipredator behavior. Behavioral Ecology, 15(4), 673 - 678.

Via, S., \& Lande, R.. (1985). Genotype-environment interaction and the evolution of phenotypic plasticity. Evolution, 39(3), 505 - 522.

Via, S.. (1987). Genetic constraints on the evolution of phenotypic plasticity. In V. Loeschcke (Ed.), Genetic Constraints on Adaptive Evolution. (pp. 47 - 71). Berlin, Germany: Springer-Verlag

Wai, E.H., and Hoar, W.S.. (1963). The secondary sex characters and reproductive behavior of gonadectomized sticklebacks treated with methyl testosterone. Canadian Journal of Zoology, 41(4), 611 - 628.

West-Eberhard, M.J. . (1989). Phenotypic plasticity and the origins of diversity. Аnnu. Rev. Ecol. Syst., 20, 249 - 78.

West-Eberhard M.J. (2003). Developmental plasticity and evolution. Oxford: Oxford University Press.

Wilson, A. D. M. and Godin, J. G. J. ( 2009). Boldness and behavioral syndromes in the bluegill sunfish, Lepomis macrochirus. Behavioral Ecology, 20, 231 - 237.

Wilson D.S., Clark A.B., Coleman K., and Dearstyne T. (1994). Shyness and boldness in humans and other animals. Trends in Ecology and Evolution, 9, 442 - 446. 
Wilson, D. S., Coleman, K., Clark, A. B. \& Biederman, L. (1993). Shy-bold continuum in pumpkinseed sunfish (Lepomis gibbosus): an ecological study of a psychological trait. Journal of Comparative Psychology, 107, 250 - 260.

Wingfield JC, Hegner RE, Dufty AM, Ball GF. (1990). The “challenge hypothesis”: theoretical implications for patterns of testosterone secretion, mating systems, and breeding strategies. American Naturalist, 136, 829 - 46.

Wong, S., Dykstra, M., Campbell, J. \& Earley, R.L.. (2008). Measuring water-borne cortisol in convict cichlids (Amatitlania nigrofasciata): is the procedure a stressor? Behaviour, 145, 1283 - 1305.

Wright KJ, Higgs DM, Cato DH, and Leis JM. (2010).Auditory sensitivity in settlementstage larvae of coral reef fishes. Coral Reefs, 29,235 - 243.

Zar, J. H. (1996). Biostatistical analysis (3rd ed.). New Jersey: Prentice Hall.

Zuk, M and Kolluru, G.R. (1998). Exploitations of Sexual Signals by Predators and Parasitoids. The Quarterly Review of Biology, 73(4), 415 - 438. 